\title{
CARMA LARGE AREA STAR FORMATION SURVEY: PROJECT OVERVIEW WITH ANALYSIS OF DENSE GAS STRUCTURE AND KINEMATICS IN BARNARD 1
}

Shaye Storm ${ }^{1}$, Lee G. Mundy ${ }^{1}$, Manuel Fernández-López ${ }^{2,3}$, Katherine I. Lee ${ }^{2,1}$, Leslie W. Looney ${ }^{2}$, Peter Teuben $^{1}$, Erik W. Rosolowsky ${ }^{4,5}$, Héctor G. Arce ${ }^{6}$, Eve C. Ostriker ${ }^{7}$, Dominique M. Segura-Cox ${ }^{2}$, Marc W. Pound $^{1}$, Demerese M. Salter ${ }^{1}$, Nikolaus H. Volgenau ${ }^{8}$, Yancy L. Shirley ${ }^{9}$, Che-Yu Chen ${ }^{1}$, Hao Gong ${ }^{1}$, Adele L. Plunkett $^{6}$, John J. Tobin ${ }^{10}$, Woojin Kwon ${ }^{11}$, Andrea Isella ${ }^{12}$, Jens Kauffmann ${ }^{13}$, Konstantinos Tassis ${ }^{14,15}$, Richard M. Crutcher ${ }^{2}$, Charles F. Gammie ${ }^{2}$, Leonardo Testi ${ }^{16}$

Accepted to The Astrophysical Journal (ApJ); August 23, 2014

(see published version for full-resolution figures)

\begin{abstract}
We present details of the CARMA Large Area Star Formation Survey (CLASSy), while focusing on observations of Barnard 1. CLASSy is a CARMA Key Project that spectrally imaged $\mathrm{N}_{2} \mathrm{H}^{+}$, $\mathrm{HCO}^{+}$, and $\mathrm{HCN}(J=1 \rightarrow 0$ transitions) across over 800 square arcminutes of the Perseus and Serpens Molecular Clouds. The observations have angular resolution near $7^{\prime \prime}$ and spectral resolution near $0.16 \mathrm{~km} \mathrm{~s}^{-1}$. We imaged $\sim 150$ square arcminutes of Barnard 1, focusing on the main core, and the B1 Ridge and clumps to its southwest. $\mathrm{N}_{2} \mathrm{H}^{+}$shows the strongest emission, with morphology similar to cool dust in the region, while $\mathrm{HCO}^{+}$and $\mathrm{HCN}$ trace several molecular outflows from a collection of protostars in the main core. We identify a range of kinematic complexity, with $\mathrm{N}_{2} \mathrm{H}^{+}$ velocity dispersions ranging from $\sim 0.05-0.50 \mathrm{~km} \mathrm{~s}^{-1}$ across the field. Simultaneous continuum
\end{abstract}

\footnotetext{
${ }^{1}$ Department of Astronomy, University of Maryland, College Park, MD 20742, USA; sstorm@astro.umd.edu

${ }^{2}$ Department of Astronomy, University of Illinois at Urbana-Champaign, 1002 West Green Street, Urbana, IL 61801, USA

${ }^{3}$ Instituto Argentino de Radioastronomía, CCT-La Plata (CONICET), C.C.5, 1894, Villa Elisa, Argentina

${ }^{4}$ University of British Columbia, Okanagan Campus, Departments of Physics and Statistics, 3333 University Way, Kelowna BC V1V 1V7, Canada

${ }^{5}$ University of Alberta, Department of Physics, 4-181 CCIS, Edmonton AB T6G 2E1, Canada

${ }^{6}$ Department of Astronomy, Yale University, P.O. Box 208101, New Haven, CT 06520-8101, USA

${ }^{7}$ Department of Astrophysical Sciences, Princeton University, Princeton, NJ 08544, USA

${ }^{8}$ Owens Valley Radio Observatory, MC 105-24 OVRO, Pasadena, CA 91125, USA

${ }^{9}$ Steward Observatory, 933 North Cherry Avenue, Tucson, AZ 85721, USA

${ }^{10}$ National Radio Astronomy Observatory, Charlottesville, VA 22903, USA

${ }^{11}$ SRON Netherlands Institute for Space Research, Landleven 12, 9747 AD Groningen, The Netherlands

${ }^{12}$ Astronomy Department, California Institute of Technology, 1200 East California Blvd., Pasadena, CA 91125, USA

${ }^{13}$ Max Planck Institut für Radioastronomie, Auf dem Hügel 69 D53121, Bonn Germany

${ }^{14}$ Department of Physics and Institute of Theoretical \& Computational Physics, University of Crete, PO Box 2208, GR-710 03, Heraklion, Crete, Greece

${ }^{15}$ Foundation for Research and Technology - Hellas, IESL, Voutes, 7110 Heraklion, Greece

${ }^{16}$ ESO, Karl-Schwarzschild-Strasse 2 D-85748 Garching bei München, Germany
} 
mapping at $3 \mathrm{~mm}$ reveals six compact object detections, three of which are new detections. A new, non-binary dendrogram algorithm is used to analyze dense gas structures in the $\mathrm{N}_{2} \mathrm{H}^{+}$positionposition-velocity (PPV) cube. The projected sizes of dendrogram-identified structures range from about 0.01-0.34 pc. Size-linewidth relations using those structures show that non-thermal lineof-sight velocity dispersion varies weakly with projected size, while rms variation in the centroid velocity rises steeply with projected size. Comparing these relations, we propose that all dense gas structures in Barnard 1 have comparable depths into the sky, around 0.1-0.2 pc; this suggests that over-dense, parsec-scale regions within molecular clouds are better described as flattened structures rather than spherical collections of gas. Science-ready PPV cubes for Barnard 1 molecular emission are available for download.

\section{INTRODUCTION}

Star formation occurs over a wide range of spatial scales and physical densities in molecular clouds. Observations reveal that molecular cloud complexes extend for several tens of parsecs, with low-density molecular gas $\left(n \leq 10^{2-3} \mathrm{~cm}^{-3}\right)$ at all spatial scales. Overdensities in the low-density gas create zones of active star formation at parsec scales with higher-density gas. These overdensities evolve to even higher densities $\left(n \geq 10^{5-7} \mathrm{~cm}^{-3}\right)$ as they form pre-stellar and proto-stellar cores on scales of $\sim 0.01-0.1 \mathrm{pc}$. The formation of initial overdensities and their subsequent evolution to even higher densities is the focus of current research; supersonic turbulent flows, magnetic fields, gravitational instabilities, and thermal-chemical processes are all involved (e.g. reviews by Mac Low \& Klessen 2004; Ballesteros-Paredes et al. 2007; McKee \& Ostriker 2007; di Francesco et al. 2007; Bergin \& Tafalla 2007; Crutcher 2012; Li et al. 2014), but the interplay between them across a broad range of size scales, and across different environments, is not fully understood.

Large area, high angular resolution surveys of dust and gas from parsec to thousand AU scales are needed to improve our understanding of the internal physical state of molecular clouds, and to follow the structure and kinematics of overdensities on the pathway to forming stars. The long term goals of these surveys are to understand what drives and controls the rate of star formation, and to answer why the stellar initial mass function has its shape and whether that shape depends on environment. We carried out a Key Project with the Combined Array for Research in Millimeter-wave Astronomy (CARMA) to address the need for large area, high resolution gas surveys. The CARMA Large Area Star Formation Survey (CLASSy) spent 700 hours imaging five large areas of star formation in the $\mathrm{N}_{2} \mathrm{H}^{+}, \mathrm{HCO}^{+}$, and $\mathrm{HCN}(J=1 \rightarrow 0)$ molecular lines. We chose these molecules because they are dense gas tracers, and potentially sample a range of chemical/physical environments within a cloud. They are all also observable in a single CARMA correlator setting.

CLASSy observed the Perseus Molecular Cloud Complex towards NGC 1333, Barnard 1, and L1451 (see Figure 1), and the Serpens Molecular Cloud towards Serpens Main and Serpens South (see Figure 2), to capture a range of star forming environments and evolutionary stages. We mapped each region with $7^{\prime \prime}$ angular resolution to resolve individual star-forming cores, and with large enough area to capture the environment in which the cores formed. Our survey data can be combined with many ancillary datasets - Spitzer catalogs of young stellar objects (YSOs), Herschel images of heating sources, Herschel and the JCMT maps of dust and continuum sources, to name a few - to provide a total picture of star forming objects and the structure and kinematics of the dense gas which is fueling their formation.

This paper presents the initial results for the Barnard 1 region. Barnard 1 is located 3.5 pc to the east of NGC 1333 in the western half of the Perseus Molecular Cloud. We assume a distance of 235 pc based on VLBI parallax measurements towards nearby sources in Perseus (SVS-13 in NGC 1333 (Hirota et al. 2008) and L1448-C in L1448 (Hirota et al. 2011)). In the context of the CLASSy campaign, we classify Barnard 1 as a moderate-activity star forming region, compared to the highly active NGC 1333 region that is dominated by a complex array of protostellar outflows, and the low-activity L1451 complex that has only one identified 
protostar (Pineda et al. 2011).

Previous surveys of the young stellar content and dust in Barnard 1 reveal a northeast-southwest oriented filament exhibiting a wide range of star formation (see Figure 3) - progressing from a tight collection of preand proto-stellar cores in the northeast "main core" that includes the well-studied B1-b and B1-c cores (Hirano et al. 1999; Matthews et al. 2006; Hiramatsu et al. 2010), to a long filament of starless gas and dust known as the B1 Ridge (Enoch et al. 2006), followed by a collection of gas and dust clumps in the southwest that includes one protostellar core and several Class II YSOs. In total, the Spitzer c2d team identified thirteen young stellar objects (YSOs) in our field (Jørgensen et al. 2006; Rebull et al. 2007; Evans et al. 2009). Twelve dust clumps were identified by Hatchell et al. (2005) using SCUBA $850 \mu \mathrm{m}$ data, while Kirk et al. (2006) identified five; the difference was attributed to different CLUMPFIND (Williams et al. 1994) thresholds. Jørgensen et al. (2007) associated an embedded YSO with five of the SCUBA dust clumps, while the rest of the dust clumps appeared starless.

This diversity of star formation activity along a single filament makes Barnard 1 a compelling region to spectrally image at high angular resolution. CLASSy adds to the large collection of work already done on Barnard 1, and provides the first large area (150 square arcminute), high angular resolution $\left(7^{\prime \prime} ; 0.008 \mathrm{pc}\right.$ at $235 \mathrm{pc})$ spectral view of the dense gas $\left(n>10^{4-6} \mathrm{~cm}^{-3}\right)$ across the entire field. This will allow us to quantify the structure and kinematics of the gas that is actively participating in the current generation of star formation in greater detail than ever before.

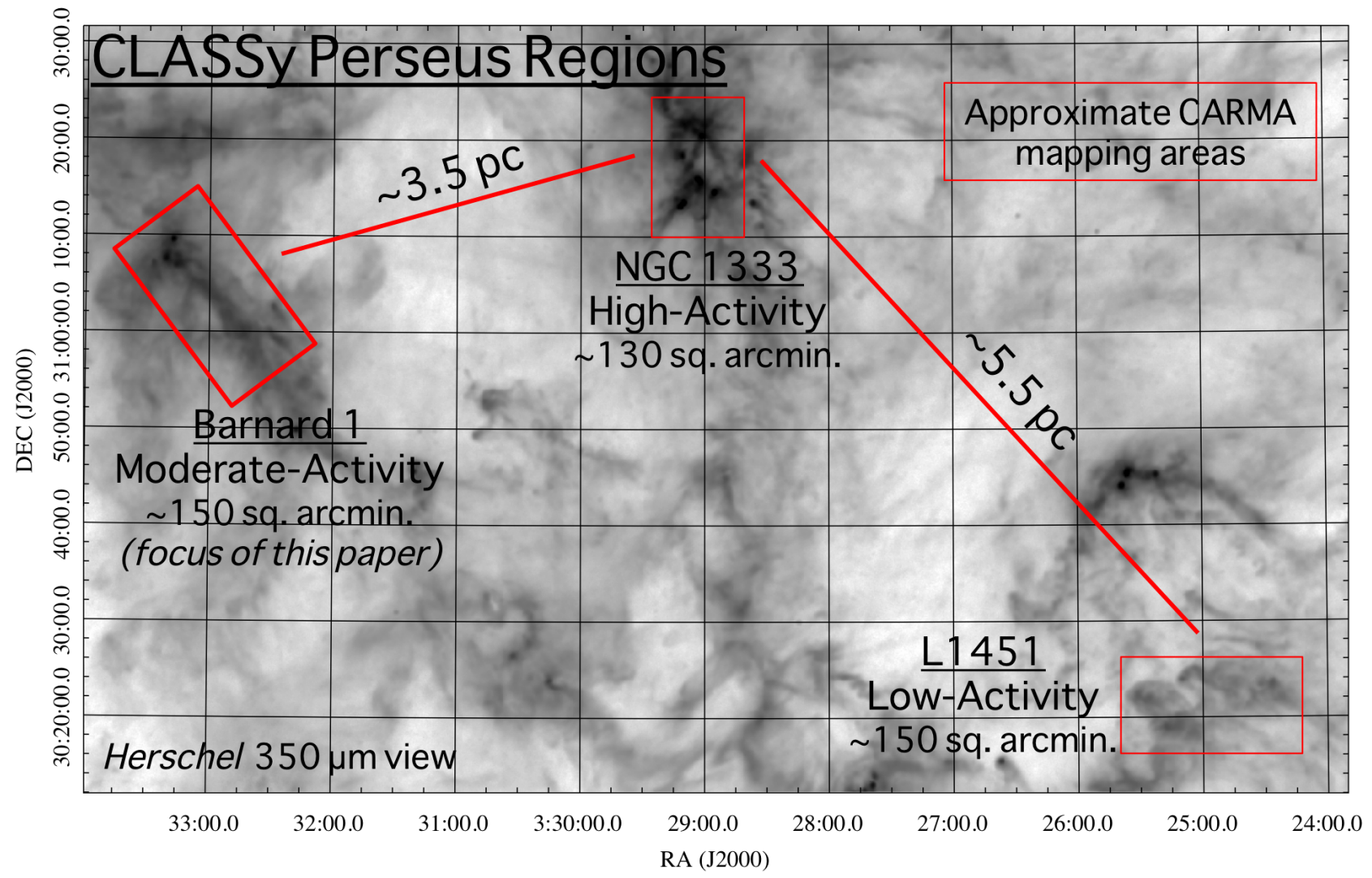

Fig. 1. - Overview of the CLASSy regions in the western portion of the Perseus Molecular Cloud, as seen by Herschel $350 \mu \mathrm{m}$ (André et al. 2010). The approximate areas mapped by CARMA are represented with rectangles (colored red in the online version); the actual areas are not single rectangular fields for Barnard 1 and L1451. Projected distances between regions are given. This paper focuses on dense gas emission in Barnard 1, and there will be a separate paper for each region. We assume a distance of 235 pc; see text. 


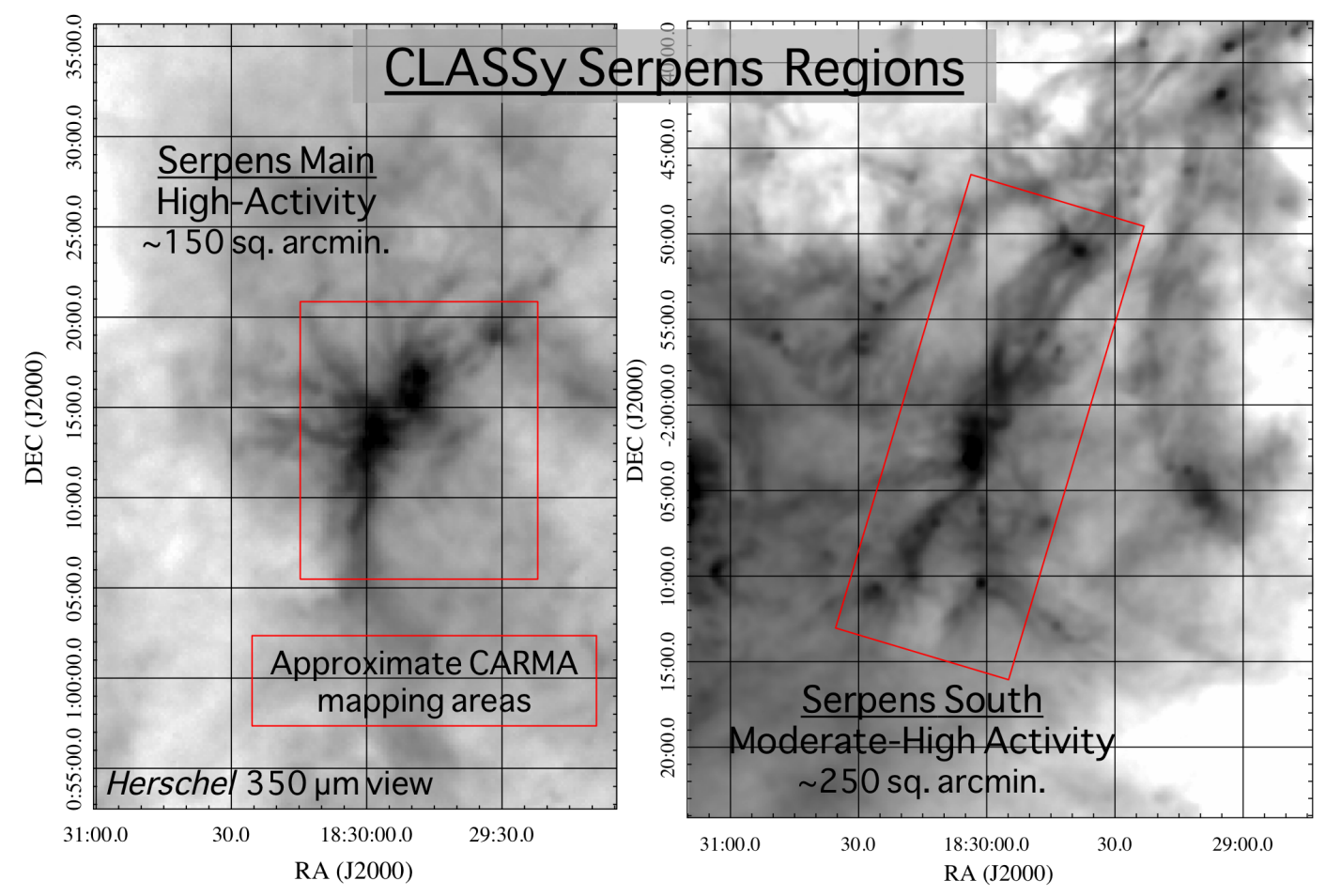

Fig. 2.- Overview of CLASSy regions in the Serpens Molecular Cloud, as seen by Herschel $350 \mu \mathrm{m}$ André et al. 2010). The approximate areas mapped by CARMA are represented with rectangles (colored red in the online version); the actual areas are not single rectangular fields for either region. The Serpens Main data is presented in Lee at al. (2014, submitted), while an analysis of the Serpens South $\mathrm{N}_{2} \mathrm{H}^{+}$filaments is in Fernández-López et al. (2014).

The primary goals of this paper are to present: (1) the details of our CLASSy observations and data calibration, (2) an overview of the dense gas morphology and kinematics in Barnard 1, and (3) a dendrogram analysis of the $\mathrm{N}_{2} \mathrm{H}^{+}$emission. In section 2, we describe the CARMA observations, data calibration, and imaging. The continuum detections are summarized in section 3. Section 4 provides a large-scale view of the dense gas structure using integrated intensity maps. In section 5, we discuss techniques for spectral line fitting and present centroid velocity and velocity dispersion maps. In section 6 , we create a dendrogram representation of the $\mathrm{N}_{2} \mathrm{H}^{+}$gas and calculate properties of identified dendrogram structures. In section 7 , we use the spatial and kinematic properties of dendrogram-identified structures to construct size-linewidth relations; these relations are then used to probe the physical and turbulent nature of Barnard 1. Section 8 has a summary of the initial results with a look ahead to upcoming science papers that utilize the data presented here. Appendix A compares clump-finding and dendrogram methods for identifying objects in this region, and Appendix B details our new dendrogram algorithm that can produce non-binary hierarchies.

\section{OBSERVATIONS}

CARMA is an ideal facility for producing molecular line maps that are sensitive to a wide range of spatial scales. The newly commissioned CARMA23 mode uses the cross-correlations from all twenty-three antennas, which increases CARMA's imaging capability from the standard CARMA15 mode. Using CARMA23 in concert with CARMA's single-dish capability and fast mosaicing can produce large maps that are sensitive to lineemission at all spatial scales. We detail the observations, data calibration, and map making in the sections below. 


\subsection{CARMA Interferometric Observations}

We mosaiced an approximately $8^{\prime} \times 20^{\prime}$ area of Barnard 1 using CARMA. The total observing time $(150$ hours) was split evenly between DZ and EZ configurations, which have projected baselines from about 1-40 k $\lambda$ and 1-30 k $\lambda$, respectively. DZ configuration observations occurred in the Spring and Fall of 2012, and EZ configuration observations occurred in the Summer of 2012. Table 1 provides a summary of the observations and calibrators. The "Z" in the DZ and EZ configurations refers to use of the 23-element observing mode (CARMA23), which utilizes cross-correlations from all 23 antennas: six 10.4-meter antennas, nine 6.1-meter antennas, and eight 3.5-m antennas. (Standard CARMA15 observing only includes the 10.4-meter and 6.1meter antennas.)

CARMA23 provides more baselines compared with the CARMA15 mode-this enhances imaging capabilities by filling in more of the $u v$-plane. It also offers shorter baselines, which are important for two reasons: (1) more extended emission, if present, is recovered, and (2) the increased $u v$-coverage improves the link between the zero spacing information from single-dish and the interferometer visibilities when doing joint deconvolution.

Our CARMA23 mosaic of Barnard 1 is made up of three adjoining rectangular regions, each at a position angle of $40^{\circ}$ east of north; it contains 743 individual pointings in a hexagonal grid with $31^{\prime \prime}$ spacing. See Figure 3 for a depiction of the pointing centers overlaid on a Herschel $250 \mu \mathrm{m}$ image (André et al. 2010). The reference position of the map, which is the center of the northern rectangle, is at $\alpha=03^{\mathrm{h}} 33^{\mathrm{m}} 20^{\mathrm{s}}, \delta=31^{\circ} 08^{\prime} 45^{\prime \prime}(\mathrm{J} 2000)$; it encompasses the Barnard 1 main core with the B1-b and B1-c continuum cores. The other two rectangles extending toward the southwest were chosen to follow the Herschel dust emission.

Table 1. Observation Summary

\begin{tabular}{lccccc}
\hline \hline Array & Dates & Total Hours & Flux Cal. & Gain Cal. & Mean Flux (Jy) \\
\hline DZ & April - May 2012 & 50 & Uranus & 3C84/3C111 & $18.3 / 3.8$ \\
& October 2012 & 25 & Uranus & 3C84/3C111 & $17.5 / 2.9$ \\
EZ & July - September 2012 & 75 & Uranus & 3C84/3C111 & $18.9 / 3.6$ \\
\hline
\end{tabular}




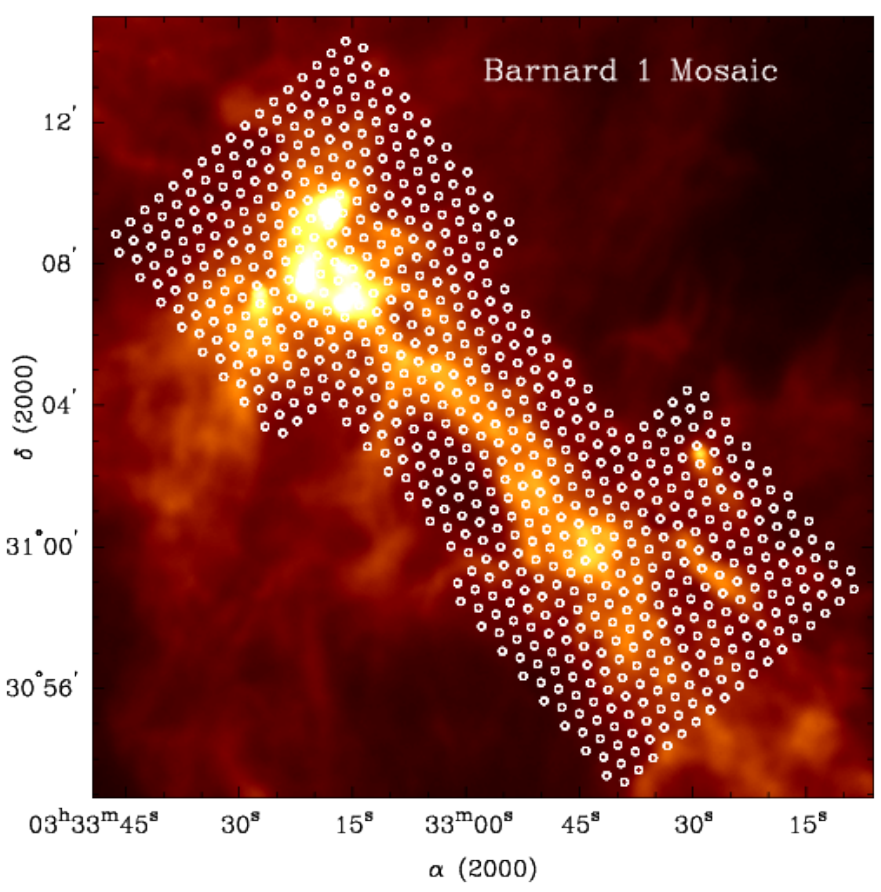

Fig. 3.- Mosaic pointing centers overlaid on a Herschel $250 \mu \mathrm{m}$ image of Barnard 1 (André et al. 2010). We sampled the field at $31^{\prime \prime}$ spacing; the smallest CARMA primary beam, from the 10.4-m antennas, is about $77^{\prime \prime}$ near $90 \mathrm{GHz}$. We planned the observations to cover the brightest dust emission in the Barnard 1 main core, and then cover the filamentary emission that extends to the southwest. Additional pointings around the edge aid in joint deconvolution by limiting strong emission at the edges of the single-dish maps.

During each pass through the map, we integrated for 15 seconds on each mosaic position before moving to the next mosaic position. We used a newly commissioned "continuous integration" technique that improves on-source efficiency (on-source integration time compared to wall clock time) of large CARMA mosaics from about $35 \%$ to $48 \%$. The improvement comes from continuously taking data, even while antennas slew to a new mosaic position; the data for a given antenna is automatically blanked while it is slewing. This technique removes the built-in software delay associated with a slew to a new target or mosaic position. The gain in onsource efficiency is critically important for large mosaic observations, with many moves and short integration times between moves. This method should not be confused with on-the-fly mosaicing, where the antennas are continuously moving while taking data.

In CARMA23 mode, the correlator has four spectral bands in the upper side band. The lower side band is not present on the 3.5-m telescopes; it is present but not used on the 10.4 and $6.1-\mathrm{m}$ telescopes. We tuned to the $J=1 \rightarrow 0$ transitions of $\mathrm{N}_{2} \mathrm{H}^{+}, \mathrm{HCO}^{+}$, and $\mathrm{HCN}$, and used a $500 \mathrm{MHz}$ wide band for calibration and continuum detections. Table 2 has an overview of the correlator setup. The molecular lines were each placed in $8 \mathrm{MHz}$ bands, which have 159 channels that are $0.049 \mathrm{MHz}$ wide in 3-bit mode; this corresponds to $\sim 0.16 \mathrm{~km} \mathrm{~s}^{-1}$ velocity resolution near $90 \mathrm{GHz}$. All hyperfine components of $\mathrm{N}_{2} \mathrm{H}^{+}$and $\mathrm{HCN}$ fit within the $8 \mathrm{MHz}$ bands. However, there is high-velocity $\mathrm{HCO}^{+}$and $\mathrm{HCN}$ emission from outflows that lies outside our bandwidth.

Two team members independently inspected, flagged, and calibrated each observing track during the 150 hour campaign using MIRIAD (Multichannel Image Reconstruction, Image Analysis and Display; Sault et al. 1995). The flags for each track were then copied to our MIS (MIRIAD Interferometric and Single-Dish; Teuben et al. 2013) pipeline that has the capability to calibrate all of the tracks with minimal user input and create combined visibility datasets and maps of all the observed sources. Standard interferometric calibration steps 
(e.g., passband, gain, flux calibration) were performed in the MIS pipeline calibration code. We observed a nearby quasar every 16 minutes for gain calibration; 3C 84 was the main gain calibrator, and 3C111 was used when $3 \mathrm{C} 84$ rose above $80^{\circ}$ elevation. 3C 84 doubled as a passband calibrator. We observed Uranus for absolute flux calibration during each track and used the bootflux task to determine the absolute flux of the gain calibrators. The flux of 3C84 varied between 16.0 and 19.4 Jy over the course of the campaign, while 3C111 varied between 2.8 and $4.5 \mathrm{Jy}$. The uncertainty in absolute flux calibration in the 24 combined datasets is about $10 \%$; hereafter, we only report statistical uncertainties in quoting errors in measured values.

\subsection{CARMA Single-Dish Observations}

Simultaneously with the interferometric observations, we obtained CARMA total power observations to recover the line emission resolved out by the interferometer (i.e., CARMA in standard interferometric observing mode). CARMA's single-dish mode utilizes the autocorrelation capability of the correlator and intersperses observations of an emission-free region between on-source integrations. A total power spectrum can then be constructed from knowledge of the system temperature $\left(\mathrm{T}_{\text {Sys }}\right)$, the emission region (the ON position), and the emission-free region (the OFF position).

The OFF position for Barnard 1 was in a gap of ${ }^{12} \mathrm{CO}$ and ${ }^{13} \mathrm{CO}$ emission $28^{\prime}$ west and $1.5^{\prime}$ south of the Barnard 1 mosaic reference position. A hole in lower density CO gas ensured that there was no significant dense gas in that region. We integrated on the OFF position for 30 seconds every 3.5 minutes if the atmospheric opacity was stable on the 3.5 minute timescale; otherwise, we observed purely in interferometric mode. Fourteen of 24 tracks with passing weather grades were observed in single-dish mode. We flagged the autocorrelation data separately from the cross-correlation data to account for cases where the opacity deteriorated in a track that was started in single-dish mode.

We calibrated the autocorrelation data from each antenna in MIRIAD with two steps: (1) sinbad calculated $\mathrm{T}_{\text {sys }} *(\mathrm{ON}-\mathrm{OFF}) /(\mathrm{OFF})$, and (2) sinpoly removed first-order polynomial baselines from the sinbad spectra. We averaged OFF scans on both sides of an ON scan in the sinbad calculation to reduce noise introduced from OFF scans.

We converted the calibrated single-dish spectra from each of the six 10.4-m antennas to six single-dish data cubes using the varmaps routine. We only used the 10.4-m antennas because they have the highest angular resolution and hence best overlap with the 3.5-m baselines in the $u v$-plane. The 10.4-m antennas have a halfpower beamwidth of $77.3^{\prime \prime}\left(\mathrm{N}_{2} \mathrm{H}^{+}\right), 80.7^{\prime \prime}\left(\mathrm{HCO}^{+}\right)$, and $81.2^{\prime \prime}(\mathrm{HCN})$. The routine gridded each data cube onto $10^{\prime \prime} \times 10^{\prime \prime}$ cells, and used a $50^{\prime \prime}$ smoothing beam to calculate the emission in each cell according to the emission at each mosaic pointing. The size of the smoothing beam was determined empirically; a smaller beam increased the final noise in the maps, while a larger beam smoothed out structure seen in other single-dish maps

Table 2. Correlator Setup Summary

\begin{tabular}{lccccccc}
\hline \hline \multicolumn{1}{c}{ Line } & $\begin{array}{c}\text { Rest Freq. } \\
(\mathrm{GHz})\end{array}$ & No. Chan. & $\begin{array}{c}\text { Chan. Width } \\
(\mathrm{MHz})\end{array}$ & $\begin{array}{c}\text { Vel. Coverage } \\
\left(\mathrm{km} \mathrm{s}^{-1}\right)\end{array}$ & $\begin{array}{c}\text { Vel. Resolution } \\
\left(\mathrm{km} \mathrm{s}^{-1}\right)\end{array}$ & $\begin{array}{c}\text { Chan. RMS } \\
\left(\mathrm{Jy} \mathrm{beam}^{-1}\right)\end{array}$ & Synth. Beam \\
& & & & & & & \\
$\mathrm{N}_{2} \mathrm{H}^{+}$ & 93.173704 & 159 & 0.049 & 24.82 & 0.157 & 0.14 & $7.6^{\prime \prime} \times 6.5^{\prime \prime}$ \\
$\mathrm{Continuum}$ & 92.7947 & 47 & 10.4 & 1547 & 33.6 & 0.0013 & $8.0^{\prime \prime} \times 6.2^{\prime \prime}$ \\
$\mathrm{HCO}^{+}$ & 89.188518 & 159 & 0.049 & 25.92 & 0.164 & 0.12 & $7.8^{\prime \prime} \times 6.8^{\prime \prime}$ \\
$\mathrm{HCN}$ & 88.631847 & 159 & 0.049 & 26.10 & 0.165 & 0.12 & $7.9^{\prime \prime} \times 6.8^{\prime \prime}$ \\
\hline
\end{tabular}

Note. - ${ }^{a}$ In principle, the synthesized beam is slightly different for each pointing. Miriad calculates a synthesized beam for the full mosaic based on all of the pointings. 
of this region. The final halfpower beamwidth of the calibrated single-dish cubes is the quadrature sum of the original halfpower beamwidth and the smoothing beam: 92.1" $\left(\mathrm{N}_{2} \mathrm{H}^{+}\right), 94.9^{\prime \prime}\left(\mathrm{HCO}^{+}\right)$, and 95.4" $(\mathrm{HCN})$.

We scaled the data cubes from all six antennas to a single reference antenna to account for systematic differences of $\sim 10 \%$ arising from physical differences in each antenna and from differences in bandpass shape of each antenna response. We calculated the mean of the six data cubes using imstack to improve the signal-tonoise ratio and limit antenna-based artifacts. The antenna temperature rms values in the final cubes are 0.025 , 0.027 , and $0.026 \mathrm{~K}$ for $\mathrm{N}_{2} \mathrm{H}^{+}, \mathrm{HCO}^{+}$, and $\mathrm{HCN}$, respectively.

\subsection{Joint Deconvolution of Interferometric and Single-Dish Cubes}

The final data product for each observed molecular line transition is a spectral line cube produced from a joint deconvolution of the interferometric and single-dish data. The joint deconvolution was done in MIRIAD as summarized below.

We created the interferometric dirty cube and dirty beam from the calibrated visibility dataset using invert with system temperature and antenna gain weighting, and Briggs' robustness parameter (Briggs et al. 1999) of -0.5 . We de-selected 10.4-m-and-3.5-m baselines due to the illumination of the first negative sidelobe of the 10.4-m beam by the 3.5-m beam. The dirty cube was then cleaned with mossdi, a Steer CLEAN algorithm, and the clean components were carried over to the joint deconvolution to aid in the convergence to a solution. The single-dish cube was regridded to the interferometric cube axes, and converted to $\mathrm{Jy} \mathrm{beam}^{-1}$ units with a $65 \mathrm{Jy}_{\text {beam }}^{-1} \mathrm{~K}^{-1}$ scaling factor 1 .

The joint deconvolution was done with a maximum entropy algorithm in MIRIAD, mosmem, that used the interferometric dirty cube, single-dish cube, interferometric dirty beam, single-dish beam, and interferometric clean components to solve for the maximum entropy model components. The final cubes are the dirty cube, minus the maximum entropy model components convolved by the dirty beam, plus the maximum entropy model components convolved by the synthesized beam. The noise levels and synthesize beams for the final data cubes are given in Table 2 .

\subsection{Continuum Mapping}

A $3 \mathrm{~mm}$ continuum map was created from the interferometric data in the $500 \mathrm{MHz}$ window. We created and cleaned the dirty map in MIRIAD using invert and mossdi, and restored with a synthesized beam of $8.0^{\prime \prime} \times 6.2^{\prime \prime}$. The $\mathrm{rms}$ in the calibrated continuum map is $\sim 1.3 \mathrm{mJy}^{\text {beam }}{ }^{-1}$.

The autocorrelation data from the $500 \mathrm{MHz}$ band was not used in continuum mapping because single-dish continuum observations require fast chopping between on-position and off-position to cancel out variable sky emission; this observing technique is not available at CARMA.

\section{CONTINUUM RESULTS}

We detected four compact continuum sources above the 5- $\sigma$ level; all are associated with young protostars in the main core. Figure 4 shows $3 \mathrm{~mm}$ continuum images toward the previously known Class 0 source, B1-c (Matthews et al. 2006), and the Class 0 double source, B1-b (Hirano et al. 1999), along with a new 5.8- $\sigma$

\footnotetext{
${ }^{1}$ http://www.mmarray.org/memos/carma_memo52.pdf
} 
detection toward a Class I source, SSTc2d J033327.3+310710 (also at the position of the lower-resolution Per-emb 30 dust source in Enoch et al. 2009).
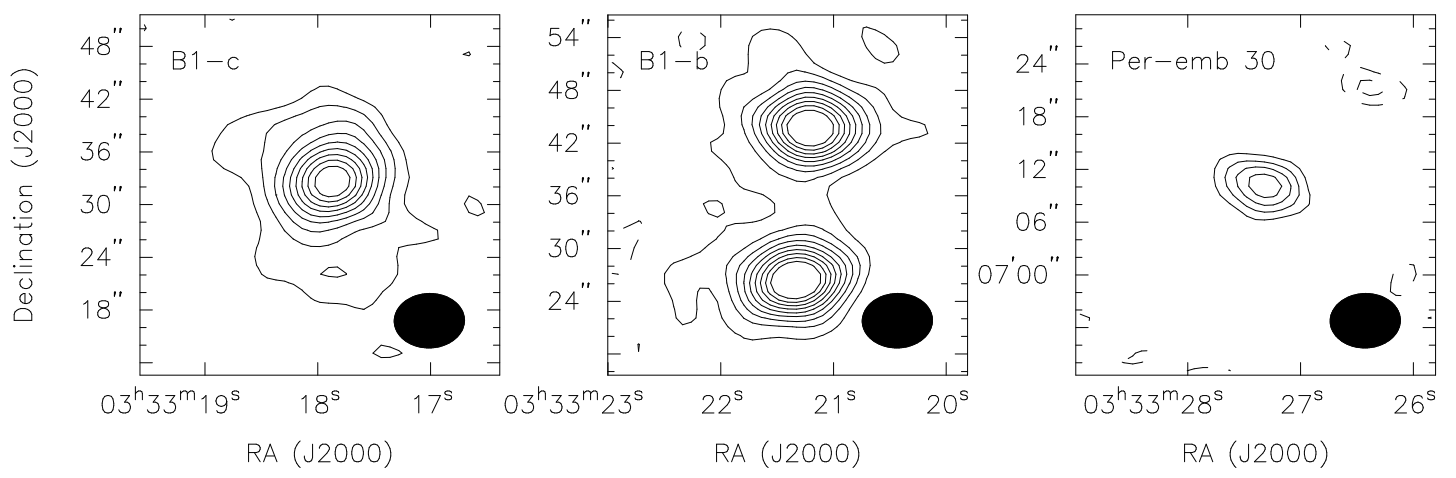

Fig. 4.- The four continuum detections above $5-\sigma$ in our field. The synthesized beam is $8.0^{\prime \prime} \times 6.2^{\prime \prime}$, and the $1-\sigma$ sensitivity is $\sim 1.3 \mathrm{mJy}_{\text {beam }}{ }^{-1}$. B1-c and B1-b were previously found to have compact continuum emission associated with young stars. The contour levels in those maps are $( \pm) 2,4,6,8,10,12,14,16,18$ times 1- $\sigma$. The compact emission within Per-emb 30 is a new detection that peaks at 5.8- $\sigma$. Contour levels in that map are $( \pm) 2,3,4,5$ times $1-\sigma$. The negative contours are represented by dashed lines.

The structure around B1-c and B1-b is clearly resolved; Per-emb 30 appears to be nearly unresolved. We used MIRIAD's imfit to determine the position, peak brightness, total flux density, size, and position angle of each source with an elliptical Gaussian approximation (see Table 3). The measured position errors are 0.2-0.7". The positions of B1-c and B1-b agree with other interferometric observations of these sources (Matthews et al. 2006: Chen et al. 2013; Huang \& Hirano 2013).

With linear sizes of 1000-2000 AU, the $3 \mathrm{~mm}$ emission is arising from compact cores associated with forming stars. In the optically thin limit, under the assumption of a single temperature, core mass is related to the total flux density as

$$
M=\frac{F_{\nu} D^{2}}{\kappa_{\nu} B_{\nu}\left(T_{\mathrm{d}}\right)}
$$

where $M, F_{\nu}, D, \kappa_{\nu}$, and $B_{\nu}\left(T_{\mathrm{d}}\right)$, are respectively the total mass, total observed flux density, distance, mass opacity including dust and gas (assuming a gas-to-dust ratio of 100), and blackbody intensity at dust temperature, $T_{\mathrm{d}}$. We assume $T_{\mathrm{d}}=20 \mathrm{~K}$. To estimate $\kappa_{\nu}$, we assumed a power law opacity curve, $\kappa_{\nu}=\kappa_{\mathrm{o}}\left(\nu / \nu_{\mathrm{o}}\right)^{\beta}$, where $\nu_{\mathrm{o}}=1000 \mathrm{GHz}$ and $\kappa_{\mathrm{o}}=0.1 \mathrm{~cm}^{2} \mathrm{~g}^{-1}$ (Beckwith et al. 1990). For a $\beta$ of $1.5, \kappa_{\nu}$ is $0.0028 \mathrm{~cm}^{2} \mathrm{~g}^{-1}$ at 92.79 GHz. The core masses under these assumptions are listed in Table 3; statistical errors are reported using the uncertainty in the total flux density. The B1-c mass estimate is about three times lower than the mass

Table 3. Observed Properties of Continuum Detections

\begin{tabular}{|c|c|c|c|c|c|c|c|c|c|}
\hline $\begin{array}{l}\text { Source } \\
\text { Name } \\
(1)\end{array}$ & $\begin{array}{c}\text { Position } \\
\left(\mathrm{h}: \mathrm{m}: \mathrm{s}, \mathrm{d}:^{\prime}:^{\prime \prime}\right) \\
(2)\end{array}$ & $\begin{array}{l}\text { Pk. Bright. } \\
\left(\mathrm{mJy} \mathrm{beam}^{-1}\right) \\
(3)\end{array}$ & $\begin{array}{c}\text { Total } \mathrm{S}_{\nu} \\
(\mathrm{mJy}) \\
(4)\end{array}$ & $\begin{array}{c}\text { Ang. Size } \\
\left(\begin{array}{l}\prime \prime \\
(5)\end{array}\right.\end{array}$ & $\begin{array}{c}\text { P.A. } \\
\left(\begin{array}{l}\circ \\
(6)\end{array}\right. \\
(6)\end{array}$ & $\begin{array}{c}\text { Decon. Size } \\
\left(\begin{array}{l}\prime \prime \\
(7)\end{array}\right. \\
(7)\end{array}$ & $\begin{array}{c}\text { Decon. P.A. } \\
\left(\begin{array}{l}{ }^{\circ} \\
(8)\end{array}\right. \\
(8)\end{array}$ & $\begin{array}{l}\text { Lin. Size } \\
\text { (AU) } \\
(9)\end{array}$ & $\begin{array}{l}\text { Mass } \\
\left(M_{\odot}\right) \\
(10)\end{array}$ \\
\hline B1-c & $03: 33: 17.91,+31: 09: 32.8$ & $23.0 \pm 1.8$ & $52.6 \pm 4.2$ & $10.7 \times 10.7$ & -33 & $8.7 \times 7.1$ & -1 & $2040 \times 1670$ & $1.04 \pm 0.08$ \\
\hline B1-b South & $03: 33: 21.37,+31: 07: 26.4$ & $29.1 \pm 2.3$ & $42.1 \pm 3.3$ & $9.8 \times 7.4$ & -79 & $5.9 \times 3.6$ & -65 & $1390 \times 850$ & $0.84 \pm 0.07$ \\
\hline B1-b North & $03: 33: 21.23,+31: 07: 43.7$ & $27.5 \pm 2.2$ & $43.0 \pm 3.3$ & $10.1 \times 7.7$ & 89 & $6.2 \times 4.5$ & 87 & $1460 \times 1060$ & $0.85 \pm 0.07$ \\
\hline Per-emb 30 & $03: 33: 27.35,+31: 07: 10.0$ & $6.9 \pm 0.8$ & $8.9 \pm 1.0$ & $9.7 \times 6.6$ & 79 & $5.5 \times 1.7$ & 69 & $1290 \times 400$ & $0.18 \pm 0.02$ \\
\hline W1 & $03: 33: 14.8,+31: 07: 13$ & $4.1 \pm 1.3$ & $\ldots$ & $\ldots$ & $\ldots$ & $\ldots$ & ... & $\ldots$ & ${ }^{a} 0.08 \pm 0.03$ \\
\hline W2 & $03: 33: 16.7,+31: 06: 53$ & $4.4 \pm 1.3$ & $\ldots$ & $\cdots$ & $\cdots$ & $\ldots$ & $\cdots$ & $\cdots$ & ${ }^{a} 0.09 \pm 0.03$ \\
\hline
\end{tabular}


reported in Matthews et al. (2006), primarily due to differences in the observed total flux density near $3.3 \mathrm{~mm}$. The B1-b mass estimates are several times larger than results from Huang \& Hirano (2013), who observed the sources at $1 \mathrm{~mm}$ with higher angular resolution; the disparity increases when adopting their assumed $\beta$ and $T_{\mathrm{d}}$ values. Since they derived deconvolved sizes of $\sim 300-500 \mathrm{AU}$, it is most likely that their mass estimates are not including extended emission in the protostellar envelope.

In addition to these relatively strong detections, we detected two continuum peaks greater than 3- $\sigma$ (labeled W1-2 in Table 3p that are coincident with a source in at least one Spitzer or Herschel band. The positions, peak brightnesses, and lower-limit masses for these sources are listed in Table 3 . We determined position using imfit with an elliptical Gaussian approximation. Peak brightness was defined as the maximum pixel value within the $3-\sigma$ contour of the source, with error equal to the 1- $\sigma$ sensitivity of the continuum map. The lower-limit mass was calculated from the peak brightness. W1 is coincident with SSTc2d J033314.3+310710 (Per-emb 6), and W2 is coincident with SSTc2d J033316.4+310652 (Per-emb 10). Deeper, follow-up observations are needed to confirm these detections and calculate physical properties of the sources.

\section{MORPHOLOGY OF DENSE MOLECULAR GAS}

Figure 5 shows our $\mathrm{N}_{2} \mathrm{H}^{+}, \mathrm{HCN}$, and $\mathrm{HCO}^{+}(J=1 \rightarrow 0)$ integrated intensity maps, along with a Herschel $250 \mu \mathrm{m}$ view of Barnard 1 for a qualitative gas-dust comparison. The angular resolution of the dust map is $18.1^{\prime \prime}$ in comparison to our $\sim 7^{\prime \prime}$ resolution. To facilitate the discussion later in this section, we identify three zones of emission: the main core zone, the ridge zone, and the SW clumps zone. Figure 6 shows example spectra from each zone at the locations marked by crosses in Figure 5 , and the next three sections discuss the three zones in detail.

The $\mathrm{N}_{2} \mathrm{H}^{+}$map was integrated over all seven hyperfine components over velocity ranges from 14.044 to $10.745 \mathrm{~km} \mathrm{~s}^{-1}, 8.860$ to $4.775 \mathrm{~km} \mathrm{~s}^{-1}$, and -0.951 to $-2.766 \mathrm{~km} \mathrm{~s}^{-1}$. The HCN map was integrated over all three hyperfine components, excluding channels with outflows, from 12.117 to $10.961 \mathrm{~km} \mathrm{~s}^{-1}, 7.326$ to

$6.005 \mathrm{~km} \mathrm{~s}^{-1}$, and 0.057 to $-1.264 \mathrm{~km} \mathrm{~s}^{-1}$. The $\mathrm{HCO}^{+}$map was integrated from 7.161 to $5.849 \mathrm{~km} \mathrm{~s}^{-1}$, again excluding outflow channels. We integrated $\mathrm{N}_{2} \mathrm{H}^{+}$over a wider range of velocities compared to the other molecules to include a narrow, redshifted filament, which can be seen along the southeastern edge of the thicker $\mathrm{N}_{2} \mathrm{H}^{+}$filament in the ridge zone. This redshifted filament, discussed later in this section, is also detected in $\mathrm{HCN}$ and $\mathrm{HCO}^{+}$, but overlaps in velocity space with $\mathrm{HCN}$ and $\mathrm{HCO}^{+}$outflow channels that were excluded from these integrated intensity maps. The rms of the $\mathrm{N}_{2} \mathrm{H}^{+}, \mathrm{HCN}$, and $\mathrm{HCO}^{+}$integrated intensity maps in Figure 5 are 0.17, 0.10, and $0.06 \mathrm{Jy}_{\text {beam }}^{-1} \mathrm{~km} \mathrm{~s}^{-1}$, respectively.

The peak brightness temperature for a single channel (including channels with outflows) in the entire field occurs towards the B1-c continuum core; it is $9.5 \mathrm{~K}, 9.7 \mathrm{~K}$, and $7.1 \mathrm{~K}$ in our synthesized beam for $\mathrm{N}_{2} \mathrm{H}^{+}$(2.85 Jy beam ${ }^{-1}$ to $\mathrm{K}$ conversion factor), $\mathrm{HCN}$ (2.91 Jy beam ${ }^{-1}$ to $\mathrm{K}$ conversion factor), and $\mathrm{HCO}^{+}$ (2.91 $\mathrm{Jy}_{\text {beam }}{ }^{-1}$ to $\mathrm{K}$ conversion factor), respectively.

\subsection{Main Core Zone}

The main core zone, shown in Figure 7, is the area of strongest dust emission seen by Herschel; it contains the largest cluster of young protostellar objects, and is the only region where we have detected compact continuum sources (see section 3) and outflows (see Figure 8). The $\mathrm{N}_{2} \mathrm{H}^{+}$emission in this zone follows the overall structure of the dust, although our improved angular resolution reveals more detail than ever before. The $\mathrm{HCN}$ and $\mathrm{HCO}^{+}$emission not associated with outflows is much weaker compared to $\mathrm{N}_{2} \mathrm{H}^{+}$, and it does not follow the morphological structure of the $\mathrm{N}_{2} \mathrm{H}^{+}$or the dust. Figure 7 shows the locations of Spitzer YSO 

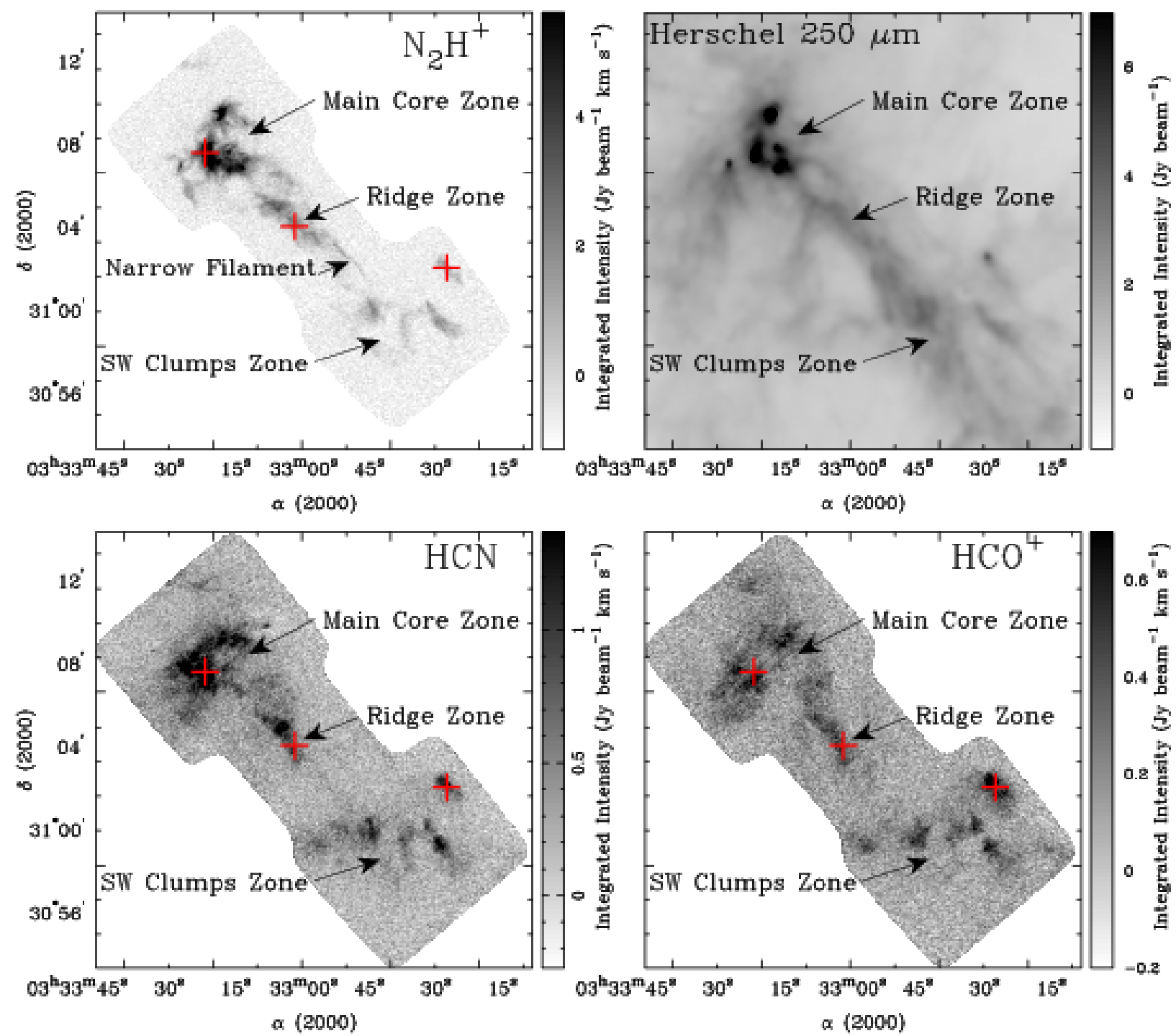

Fig. 5.- Integrated intensity maps of $\mathrm{N}_{2} \mathrm{H}^{+}, \mathrm{HCN}$, and $\mathrm{HCO}^{+}(J=1 \rightarrow 0)$ emission towards Barnard 1 , with a Herschel $250 \mu \mathrm{m}$ map. Channels containing outflow emission are excluded from these maps, which is why the narrow filament (see Section 4.2) appears only in $\mathrm{N}_{2} \mathrm{H}^{+}$. The rms values of the $\mathrm{N}_{2} \mathrm{H}^{+}, \mathrm{HCN}$, and $\mathrm{HCO}^{+}$maps are $0.17,0.10$, and $0.06 \mathrm{Jy} \mathrm{beam}^{-1} \mathrm{~km} \mathrm{~s}^{-1}$, respectively. We break the region into three zones based on qualitative features in the dense gas maps. Crosses in the maps represent the locations of spectra shown in Figure 6. FITS cubes of the PPV data used to make this figure are available in the online journal. 

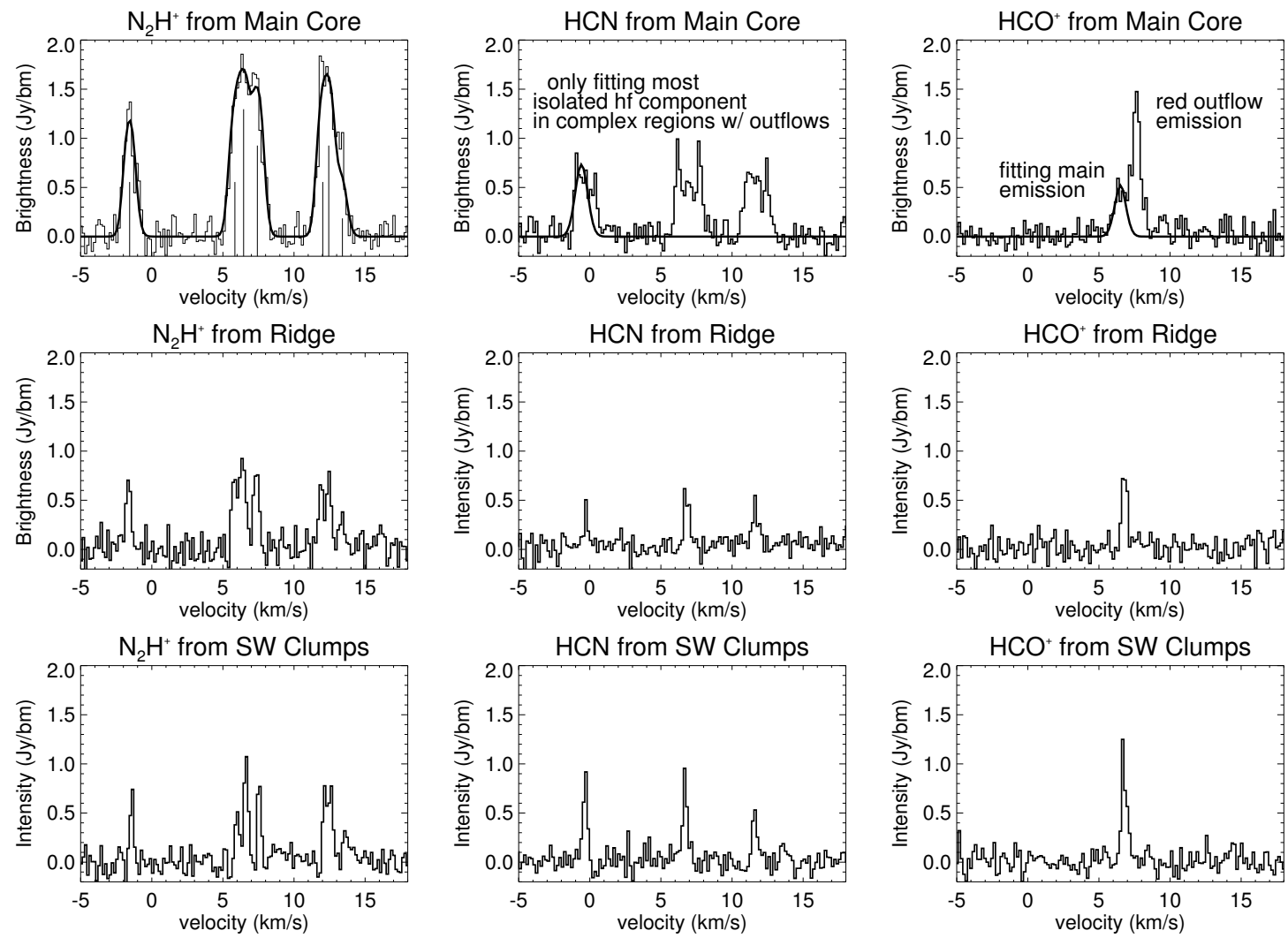

Fig. 6. - Example spectra for all three molecules from each zone. The spectra are averaged over one synthesized

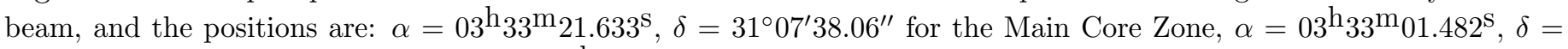

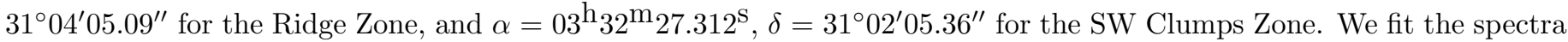
and present maps of centroid velocity and velocity dispersion in section 5 . The fits to the main core zone spectra are shown with solid black lines. The $\mathrm{N}_{2} \mathrm{H}^{+}$has seven resolvable hyperfine components in narrow-line regions, and HCN has three resolvable hyperfine components. The hyperfine structure of $\mathrm{N}_{2} \mathrm{H}^{+}$is shown with vertical bars set within the main core zone spectrum - the relative brightnesses are representative of the hyperfine structure, but the absolute values were picked for ease of visualization. As described in section 5, we exclude outflow emission from $\mathrm{HCN}$ and $\mathrm{HCO}^{+}$when doing these fits. 
candidates (Jørgensen et al. 2006), Bolocam $1 \mathrm{~mm}$ clumps (Enoch et al. 2006), and SCUBA $850 \mu \mathrm{m}$ clumps (Hatchell et al. 2005).

The spectra from this zone are the most complex in the Barnard 1 field - Figure 6 shows a sample $\mathrm{N}_{2} \mathrm{H}^{+}$ spectrum from near the B1-b core that exhibits broad lines, while the $\mathrm{HCN}$ and $\mathrm{HCO}^{+}$spectra from the same location show evidence of red-wing outflow emission overlapping with B1-b. The $\mathrm{N}_{2} \mathrm{H}^{+}$emission towards B1-b shows two resolved peaks. There are emission peaks in $\mathrm{HCN}$ and $\mathrm{HCO}^{+}$towards B1-b South, but not B1-b North (see Figure 7). This agrees with findings from Huang \& Hirano (2013) and supports the idea that carbon-bearing species are depleted around B1-b North, suggesting it is the younger source of the two.

The $\mathrm{N}_{2} \mathrm{H}^{+}$gas emission is very strong at the location of B1-c, while $\mathrm{HCO}^{+}$is only detected in outflows; this agrees with observations from Matthews et al. (2006). HCN gas does exist at the location of B1-c, but is not a strong peak relative to the rest of the main core, like we see with $\mathrm{N}_{2} \mathrm{H}^{+}$. $\mathrm{HCN}$ is also detected in outflow emission.

There are seven Spitzer Class 0/I YSOs in this zone. Two are known to be associated with the B1-c and Per-emb 30 compact continuum cores, which are sites of strong $\mathrm{N}_{2} \mathrm{H}^{+}$emission. There are four nearby the larger scale B1-a, B1-b, and B1-d dust clumps, which are also areas of strong $\mathrm{N}_{2} \mathrm{H}^{+}$emission. The seventh YSO, which lies west of the strongest dense gas in this zone, only has weak dust and gas emission associated with it. Several of these YSOs are driving outflows, which are discussed later in this section, and identified in Figure 8 and Table 4.

There are five Bolocam $1.1 \mathrm{~mm}$ cores and six SCUBA $850 \mu \mathrm{m}$ cores in this region. All of these lower resolution dust cores are near $\mathrm{N}_{2} \mathrm{H}^{+}$emission peaks, but not necessarily near $\mathrm{HCN}$ or $\mathrm{HCO}^{+}$peaks. There are several peaks of $\mathrm{HCN}$ and $\mathrm{HCO}^{+}$that are not associated with strong dust emission. 

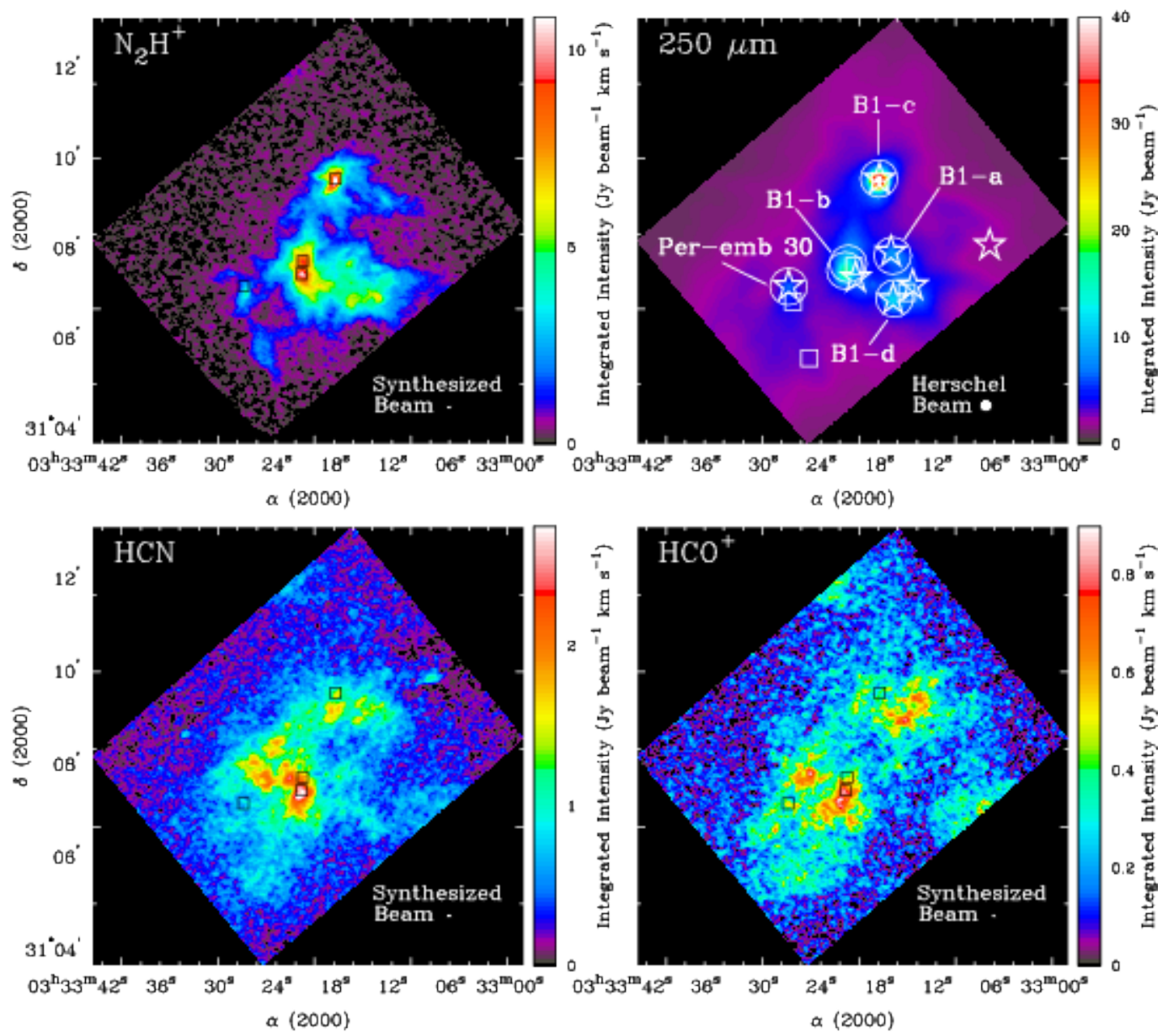

Fig. 7.- Integrated intensity maps of $\mathrm{N}_{2} \mathrm{H}^{+}, \mathrm{HCN}$, and $\mathrm{HCO}^{+}(J=1 \rightarrow 0)$ emission toward the main core zone, along with Herschel $250 \mu \mathrm{m}$. Channels containing outflow emission are excluded from the integrated intensity maps. The angular resolution of each map is marked with a beam in the lower right corner - the CARMA synthesized beam is $\sim 7^{\prime \prime}$. The Herschel map shows the location of Spitzer YSOs (stars), Bolocam $1.1 \mathrm{~mm}$ cores (squares), and SCUBA $850 \mu \mathrm{m}$ cores (circles); all YSOs in this region are Class 0/I. Major dust clumps mentioned in the text are labeled in the Herschel map. The black boxes in the integrated intensity maps show the locations of the compact continuum sources that were discussed in section 3. The rms of the $\mathrm{N}_{2} \mathrm{H}^{+}, \mathrm{HCN}$, and $\mathrm{HCO}^{+}$integrated intensity maps are $0.17,0.10$, and $0.06 \mathrm{Jy}_{\text {beam }}{ }^{-1} \mathrm{~km} \mathrm{~s}^{-1}$, respectively.

Figure 8 shows $\mathrm{HCO}^{+}$and $\mathrm{HCN}$ dense gas outflows, which are only detected in this zone. In the $\mathrm{HCO}^{+}$ map, SSTc2d J033317.9+31092 (B1-c) is clearly driving a bipolar outflow. We detect another bipolar $\mathrm{HCO}^{+}$ outflow, likely associated with the IRAS 03301+3057 YSO based on a similar detection in CO by Hirano et al. (1997). There is a red outflow just to the west of SSTc2d J033314.4+310711, another to the north SSTc2d J033320.3+310721, and a third to the southeast of SSTc2d J033327.3+310710.

In the HCN map, we again see the bipolar outflow from SSTc2d J033317.9+31092 (B1-c), although the HCN additionally traces an extension of the red part of the outflow to the west, and an associated knot of emission to the east. The western extension flares out into two separate, high-velocity outflows that extend beyond the edge of our spectral window. The eastern knot is likely a bright bow shock from the edge of the outflow interacting with cloud material. We confirmed the association of these extended features with the main outflow using Spitzer images that trace the shocked gas. The outflows from IRAS 03301+3057 and SSTc2d J033320.3+310721 are also detected in HCN, while those from J033314.4+310711 and J033327.3+310710 are 
not.
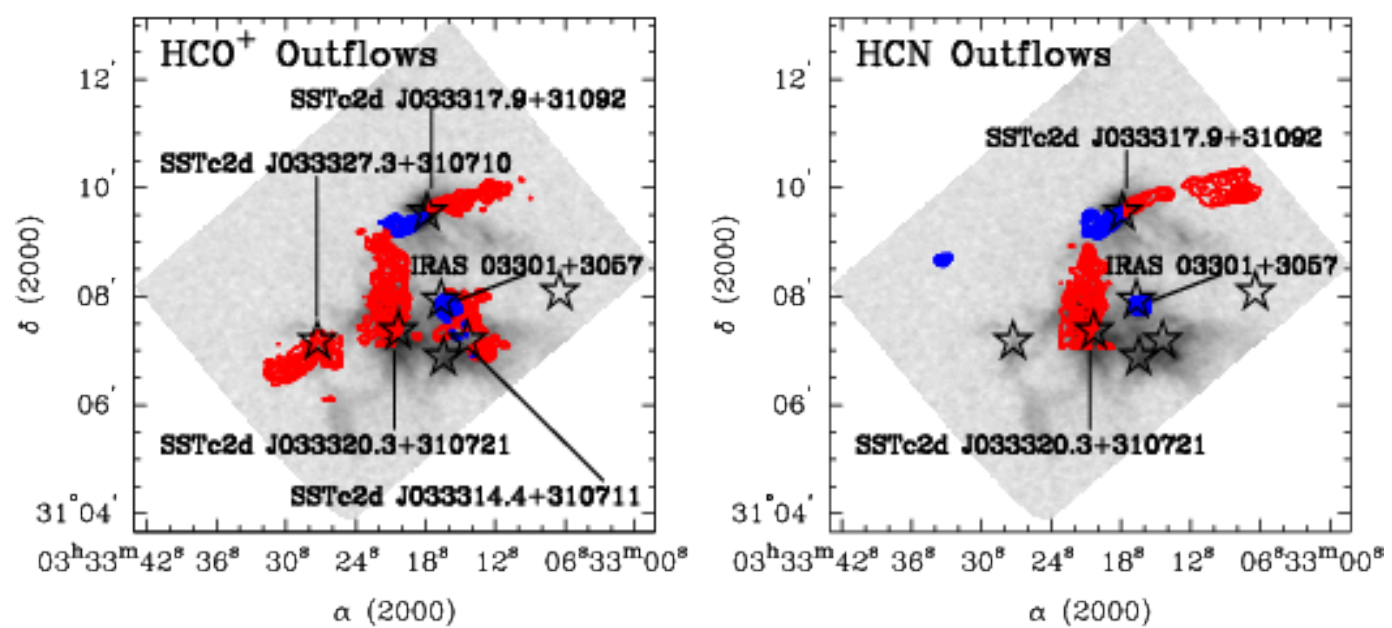

Fig. 8. - $\mathrm{HCO}^{+}$and $\mathrm{HCN}$ outflows overlaid on $\mathrm{N}_{2} \mathrm{H}^{+}$integrated intensity emission. Each outflow was integrated over its own range of velocities to minimize noise introduced from emission-free channels. We are plotting contours of $0.3,0.4$, 0.5, 0.6, 0.7, 0.8, 0.9 times the peak integrated intensity of the outflow, except for the HCN bipolar outflow from SSTc2d J033317.9+31092 where we extend down to 0.1 and 0.2 times the peak. See Table 4 for the velocity ranges and peak integrated intensity of each outflow - the outflows are identified in the table by the nearest infrared source plotted in this figure. Spitzer YSO positions are indicated with black stars; all YSOs are Class 0/I in this region. 
Table 4. Outflow Identification

\begin{tabular}{|c|c|c|c|}
\hline Source Identifier $^{a}$ & $\begin{array}{c}\text { Color } \\
\text { (Red or Blue) }\end{array}$ & $\begin{array}{l}\text { Integrated Velocity Range } \\
\qquad\left(\mathrm{km} \mathrm{s}^{-1}\right)\end{array}$ & $\begin{array}{l}\text { Peak Integrated Intensity } \\
\quad\left(\mathrm{Jy}_{\mathrm{b}} \mathrm{bam}^{-1} \mathrm{~km} \mathrm{~s}^{-1}\right)\end{array}$ \\
\hline \multicolumn{4}{|c|}{$\mathrm{HCO}^{+}$} \\
\hline SSTc2d J033317.9+310932 (B1-c, Per-emb 29) & Red & $9.62-7.98$ & 1.0 \\
\hline SSTc2d J033317.9+310932 (B1-c, Per-emb 29) & Blue & $5.36-3.72$ & 1.9 \\
\hline IRAS 03301+3057 (B1-a, Per-emb 40) & Red & $7.66-7.49$ & 0.5 \\
\hline IRAS 03301+3057 (B1-a, Per-emb 40) & Blue & $5.36-1.26$ & 2.4 \\
\hline SSTc2d J033327.3+310710 (Per-emb 30) & Red & $7.82-7.32$ & 0.8 \\
\hline SSTc2d J033320.3+310721 (Per-emb 41) & Red & $9.95-7.33$ & 1.8 \\
\hline SSTc2d J033314.4+310711 (Per-emb 6) & Red & $13.73-7.82$ & 4.4 \\
\hline \multicolumn{4}{|c|}{$\mathrm{HCN}$} \\
\hline SSTc2d J033317.9+310932 (B1-c, Per-emb 29) & Red & $18.89-11.78,10.13-7.66$ & 12.0 \\
\hline SSTc2d J033317.9+310932 (B1-c, Per-emb 29) & Blue & $10.63-7.66,5.67-1.54,(-) 1.26-(-) 3.08$ & 10.1 \\
\hline SSTc2d J033317.9+310932 (B1-c, Per-emb 29) & Blue $^{b}$ & $(-) 1.26-(-) 5.72$ & 3.3 \\
\hline IRAS 03301+3057 (B1-a, Per-emb 40) & Blue & $10.30-9.64,5.34-4.19$ & 2.1 \\
\hline SSTc2d J033320.3+310721 (Per-emb 41) & Red & $12.61-12.28,8.48-7.33,0.72-0.22$ & 1.5 \\
\hline
\end{tabular}
Note. - (a) Outflows identified by the location of the nearest infrared source. Supplemental Per-emb source identifiers are from Enoch et al. 2009 .
(b) This emission corresponds to the blue knot seen in the HCN map of Figure 8 east of the main part of the SSTc2d J0333317.9+310932 outflow. 


\subsection{Ridge Zone}

The ridge zone, shown in Figure 9, lies southwest of the main core. The most striking feature is the backbone of the B1 Ridge that appears as a structure ("Ridge" in the figure) that measures approximately $260^{\prime \prime}$ long by $80^{\prime \prime}$ wide in the $\mathrm{N}_{2} \mathrm{H}^{+}$map. This corresponds to about $0.30 \mathrm{pc}$ long by $0.09 \mathrm{pc}$ wide at a distance of 235 pc. This structure has several Bolocam and SCUBA cores running along its spine, which are strongly clustered in the northeast half where gas emission from all molecules is the strongest. A single Class 0/I YSO sits at the northeast edge of the structure - none of the dust cores along the structure have associated protostars, and they are considered pre-stellar. There is a single Bolocam core at the southwestern end of the structure, where the Herschel map peaks in an area with little molecular emission; multi-wavelength Herschel images show that this region is brighter at shorter wavelengths compared to the northeastern section of the structure. This means it could be a region of slightly higher temperature, which would explain a lower abundance of dense molecular gas. Figure 6 shows sample spectra from along this structure.

A second molecular feature in this zone is a newly discovered filament that runs parallel to the main ridge ("Narrow Fil" in Figure 9p. This filament is extremely narrow and is offset from the rest of the gas in velocity space by about $1.5 \mathrm{~km} \mathrm{~s}^{-1}$. Figure 10 shows molecular line contours overlaid on a Herschel $250 \mu \mathrm{m}$ map. The filament is about $20^{\prime \prime}$ wide and $2.5^{\prime}$ long, with a small kink half-way along its length. At a distance of $235 \mathrm{pc}$, this corresponds to $0.022 \mathrm{pc}$ wide by $0.17 \mathrm{pc}$ long. The peak integrated intensity of the filament is 1.2 $\mathrm{Jy} \mathrm{beam}^{-1} \mathrm{~km} \mathrm{~s}^{-1}, 1.4 \mathrm{Jy}_{\text {beam }}{ }^{-1} \mathrm{~km} \mathrm{~s}^{-1}$, and $0.9 \mathrm{Jy} \mathrm{beam}^{-1} \mathrm{~km} \mathrm{~s}^{-1}$, for $\mathrm{N}_{2} \mathrm{H}^{+}, \mathrm{HCN}$, and $\mathrm{HCO}^{+}$, respectively. It is not possible to identify the filament in Herschel maps because it is extremely narrow and lies along the same line of sight as the western edge of the B1 Ridge. We briefly discuss the kinematics and possible formation mechanisms of the filament in section 5 .

The third molecular feature is gas in the northern part of the zone that provides a tenuous link between the main ridge structure and the main core zone. A Class 0/I YSO is located off the southwestern edge of this feature, but there are no dust condensations directly associated with it.

\subsection{SW Clumps Zone}

The SW clumps zone lies southwest of the B1 Ridge, and is shown in Figure 11. There are five distinct clumps of $\mathrm{N}_{2} \mathrm{H}^{+}$emission, with a sixth clump only detected in $\mathrm{HCN}$ and $\mathrm{HCO}^{+}$. There are three Class II YSOs in this zone that do not correlate with any gas or dust peaks, which is expected for more evolved protostars. There is one Class 0/I YSO in this zone that lies at the northeast tip of the western-most clump. The strongest integrated $\mathrm{HCO}^{+}$emission across the entire Barnard 1 field is located in this clump-Figure 6 shows sample spectra from it. The gas morphology of this zone is very different from the main core and ridge zones. Those zones have dense gas peaks embedded within lower-intensity, larger-scale dense gas structures, while the dense gas peaks in this zone are not joined at weaker emission levels.

\section{KINEMATICS OF DENSE MOLECULAR GAS}

We modeled the spectra in our position-position-velocity (PPV) cubes to derive the centroid velocity and velocity dispersion for the emission from each molecule along every line of sight.

The $\mathrm{N}_{2} \mathrm{H}^{+}(J=1 \rightarrow 0)$ line is made up of seven resolvable hyperfine components. We model all components simultaneously and assume they are Gaussians with the same dispersion and excitation conditions. The line 

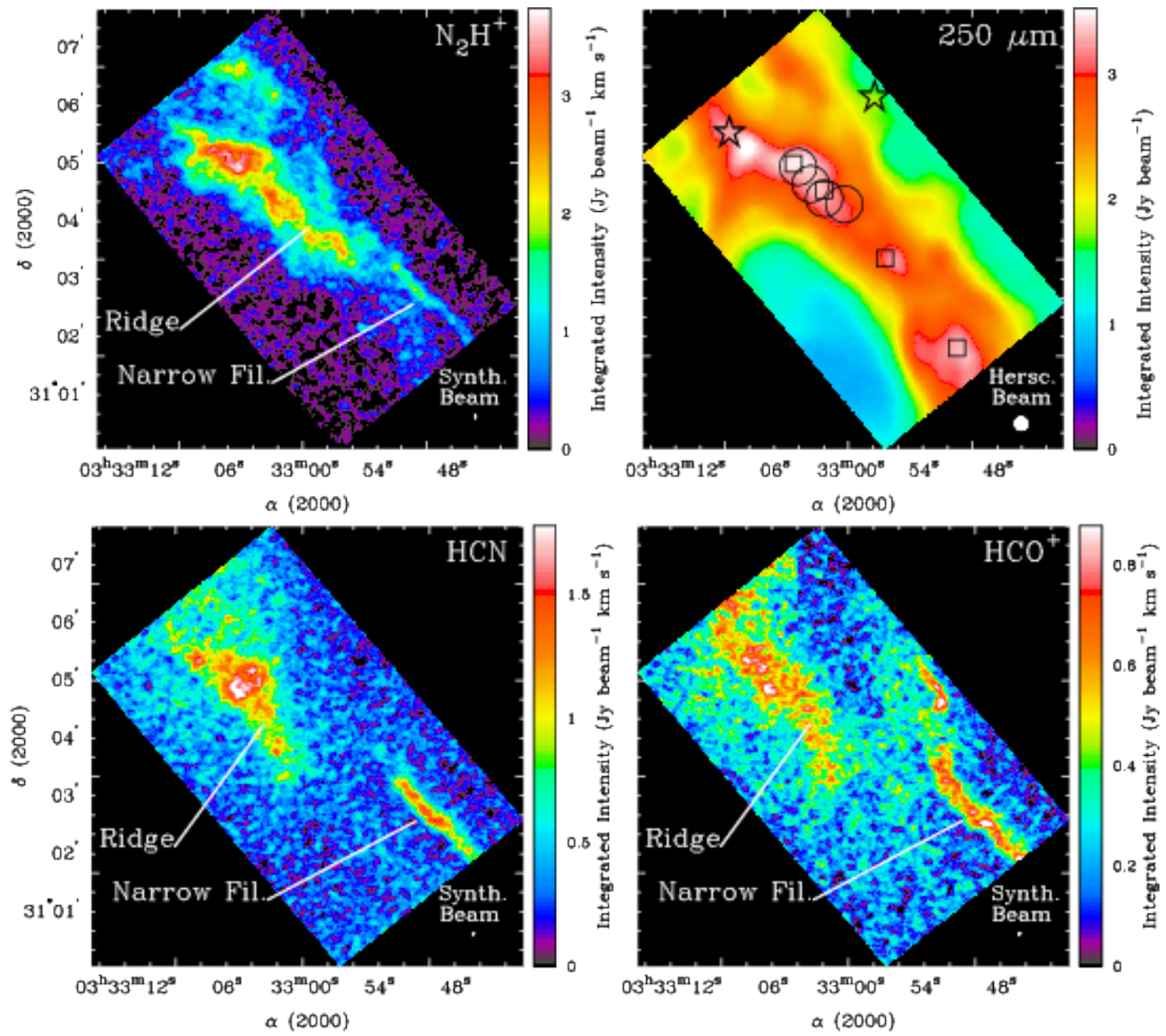

Fig. 9.- Integrated intensity maps as in Figure 7, but for the ridge zone. Since the ridge zone does not have outflows, we included extra channels that were excluded from Figure 7 to capture the narrow filament that is redshifted by $\sim 1.5 \mathrm{~km} \mathrm{~s} \mathrm{~s}^{-1}$ from the rest of the Barnard 1 gas. The rms values of these $\mathrm{N}_{2} \mathrm{H}^{+}, \mathrm{HCN}$, and $\mathrm{HCO}^{+}$integrated intensity maps are 0.17 , 0.12 , and $0.08 \mathrm{Jy}_{\text {beam }}{ }^{-1} \mathrm{~km} \mathrm{~s}^{-1}$, respectively.

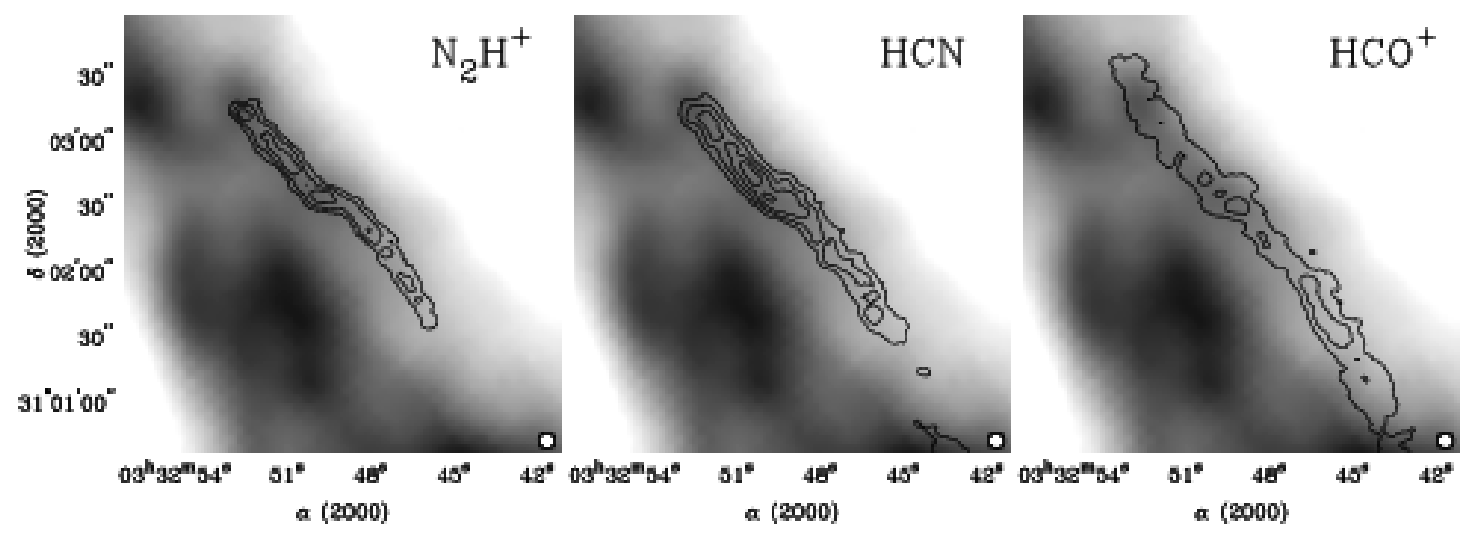

Fig. 10.- We discovered a narrow filament that is offset by $1.5 \mathrm{~km} \mathrm{~s}^{-1}$ from the rest of the gas in Barnard 1. The three figures show the filament detected in $\mathrm{N}_{2} \mathrm{H}^{+}, \mathrm{HCN}$, and $\mathrm{HCO}^{+}$, overlaid on the Herschel $250 \mu \mathrm{m}$ dust image. The contours represent integrated intensity emission at $0.3,0.5,0.7$, and 0.9 times the peak integrated intensity of the HCN filament, which is $1.36 \mathrm{Jy}_{\text {beam }}^{-1} \mathrm{~km} \mathrm{~s}^{-1}$. 

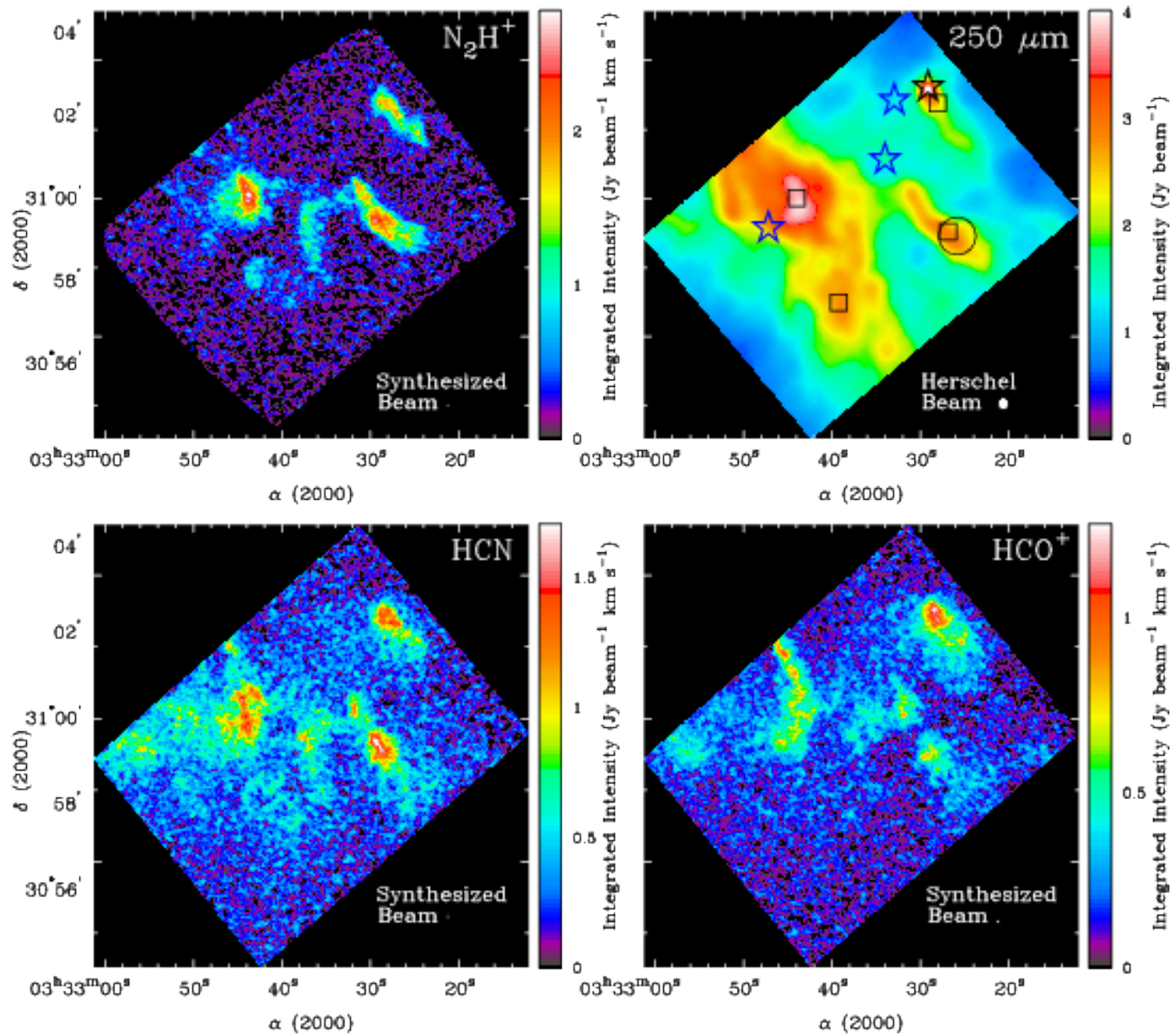

Fig. 11. - Same as Figure 7, but for the SW clumps zone. The most western YSO in this zone is a Class 0/I source, while the others are Class II sources (colored blue in the online version). Like Figure 9, since this zone does not have outflows, we included extra channels to capture the southwestern edge of the narrow filament (best seen in the $\mathrm{HCO}^{+}$ map) that is redshifted by $\sim 1.5 \mathrm{~km} \mathrm{~s}^{-1}$ from the rest of the Barnard 1 gas. The rms values of these $\mathrm{N}_{2} \mathrm{H}^{+}, \mathrm{HCN}$, and $\mathrm{HCO}^{+}$integrated intensity maps are $0.17,0.12$, and $0.08 \mathrm{Jy}_{\text {beam }}^{-1} \mathrm{~km} \mathrm{~s}^{-1}$, respectively. 
opacity as a function of velocity, $\tau(v)$, is modeled as:

$$
\tau(v)=\tau \sum_{i=0}^{7} C_{i} e^{-\left(v-V_{\mathrm{lsr}}-v_{i}\right)^{2} / 2 \sigma^{2}},
$$

where $\tau$ is the total opacity of the emission, $C_{i}$ is the weighted strength of the $i^{\text {th }}$ hyperfine component such that the sum of component strength is unity, $\mathrm{V}_{\mathrm{lsr}}$ is the centroid velocity of the observed emission, $v_{i}$ is the rest velocity of the $i^{t h}$ hyperfine component, and $\sigma$ is the velocity dispersion of each hyperfine component. We assigned $C_{i}$ and $v_{i}$ according to the CDMS catalog listings on the Splatalogue webpag $\oint^{2}-v_{i}$ was calculated from the listed $\nu_{i}$ using the rest frequency of our observations and the radio Doppler formula. A full observed spectrum is then modeled as

$$
F(v)=\frac{2 k \nu^{2} \Omega(v)}{c^{2}} \times T_{\mathrm{ex}}\left(1-e^{-\tau(v)}\right)
$$

where $k$ is Boltzmann's constant, $\Omega$ is the synthesized beam area, $v$ is the velocity of the observations, and $T_{\mathrm{ex}}$ is the excitation temperature.

The four free parameters for the fit are: $\mathrm{V}_{\mathrm{lsr}}, \sigma, \tau$, and $T_{\mathrm{ex}}$. We focus on the two kinematic parameters in the following sections. We do not address $\tau$ and $T_{\text {ex }}$ because they are semi-degenerate, and breaking the degeneracy comes from an accurate understanding of the optical depth, which is beyond the scope of this initial paper.

In the line fitting procedure, a model spectrum is first created from Equations 2 and 3 with ten times the velocity resolution of our observed data. We then bin this high-velocity-resolution model spectrum according to our observations' velocity resolution, and compare that channelized model spectrum to an observed line. The fitting is done in IDL with the MPFIT package (Markwardt 2009), which performs non-linear least-squares minimization of the model fit to an observed spectrum - the outputs are the best-fit values and errors of the four free parameters. Figure 12 (top) shows the best-fit centroid velocity $\left(\mathrm{V}_{\text {lsr }}\right)$ and velocity dispersion $(\sigma)$ maps for $\mathrm{N}_{2} \mathrm{H}^{+}$. The pixels that have data values in these maps represent spectra that pass two robustness criteria: peak signal-to-noise greater than five, and integrated intensity greater than four times the rms of the $\mathrm{N}_{2} \mathrm{H}^{+}$integrated intensity map.

The vast majority of the field only contains one resolved velocity component along each line-of-sight, and can be adequately fit with the procedure described above. However, we manually inspected the field and identified four locations with strong evidence for having two resolved velocity components. Three of those locations are marked with white rectangles in Figure 12, and are excluded from the analysis later in the paper. The fourth location is the northeastern part of the narrow filament in the ridge zone; we manually inspected this region and removed the small number of pixels with confused spectra from the $\mathrm{N}_{2} \mathrm{H}^{+}$maps.

The $\mathrm{HCN}$ and $\mathrm{HCO}^{+}$emission was fit in a similar way; the $\mathrm{HCN}$ line is made up of three resolvable hyperfine components, and $\mathrm{HCO}^{+}$has no hyperfine structure. Complications arose from outflows in the main core zone. For lines of sight complicated by outflows, we masked channels with outflow emission before fitting the spectral line representing the non-outflow gas. This was only done if the outflow emission was clearly defined in velocity space - we did not fit lines where outflow emission blended with emission nearest to the centroid velocity of the cloud. For HCN lines complicated by outflows, we only fit the highest frequency hyperfine component as it is more isolated in frequency/velocity space from the other two hyperfine components.

The middle and bottom rows of Figure 12 show the best-fit centroid velocity and velocity dispersion maps for $\mathrm{HCN}$ and $\mathrm{HCO}^{+}$, respectively. The integrated intensity criterion is the same as above. However, the peak signal-to-noise criterion is eight for $\mathrm{HCN}$, and ten for $\mathrm{HCO}^{+}$, to account for increased uncertainty in fit values

\footnotetext{
${ }^{2}$ http://splatalogue.net
} 
with decreasing number of hyperfine components. It is clear from the sparseness of the maps that there are far fewer $\mathrm{HCN}$ and $\mathrm{HCO}^{+}$regions strong enough to get reliable kinematic measurements compared to $\mathrm{N}_{2} \mathrm{H}^{+}$. The $\mathrm{HCO}^{+}$and $\mathrm{HCN}$ data also show evidence for two velocity components in the same regions as $\mathrm{N}_{2} \mathrm{H}^{+}$.

Analyzing all the details of these kinematic maps is beyond the scope of this paper. However, some features to point out are as follows:

1. The most kinematic complexity exists in the main core zone - it has velocity gradients up to $\sim 10 \mathrm{~km} \mathrm{~s}^{-1} \mathrm{pc}^{-1}$ (see Figure 13, left), and velocity dispersions ranging from $0.05 \mathrm{~km} \mathrm{~s}^{-1}$ all the way up to $0.5 \mathrm{~km} \mathrm{~s}^{-1}$. In comparison, the gas structures in the ridge and SW clump zones show smaller variations in centroid velocity and velocity dispersion, and the velocity dispersions are consistently narrower than those found in the main core zone.

2. The narrow filament in the ridge zone, which can be seen in the all three kinematic maps, is redshifted by $1.5 \mathrm{~km} \mathrm{~s}^{-1}$ relative to the rest of the Barnard 1 emission. The mean velocity dispersion along the filament is $0.12 \mathrm{~km} \mathrm{~s}^{-1}, 0.16 \mathrm{~km} \mathrm{~s}^{-1}$, and $0.20 \mathrm{~km} \mathrm{~s}^{-1}$, for $\mathrm{N}_{2} \mathrm{H}^{+}, \mathrm{HCN}$, and $\mathrm{HCO}^{+}$, respectively. At an assumed kinetic temperature of $11 \mathrm{~K}$ based on GBT data (Rosolowsky et al. 2008a), $\mathrm{N}_{2} \mathrm{H}^{+}$and $\mathrm{HCN}$ are detecting subsonic gas motions and $\mathrm{HCO}^{+}$is approaching the sonic speed. The $1.5 \mathrm{~km} \mathrm{~s}^{-1}$ radial velocity of the filament gas relative to the bulk Barnard 1 gas suggests that it did not simply fragment from the main reservoir of gas in the region. It is possible that a nearby flow of material piled onto the larger filament and flowed around its western edge; this would cause a column density increase along that edge, thereby strengthening the molecular emission and causing redshift. One problem with this scenario is that we might expect more turbulent linewidths for gas involved in a colliding flow. However, this type of flow event could have happened long enough ago that gravity had time to collect the gas into the filament we now see. Another possibility is that we are viewing a sheet-like structure edge on.

3. The largest structure in the ridge zone has a velocity gradient perpendicular to its major axis (see Figure 13 , right), which is a feature common in numerical simulations of filament formation from planar converging, turbulent flows (Chen \& Ostriker 2014). This type of velocity gradient is seen in several CLASSy filaments across our five regions; Serpens South examples are highlighted in Fernández-López et al. (2014), and we are preparing a paper linking these observations with the numerical simulations (Mundy et al., in preparation).

4. Upon close inspection, several regions of the cloud have well-organized $\mathrm{N}_{2} \mathrm{H}^{+}$centroid velocity fieldsfor example, the dense gas around B1-c shows clear signatures of envelope rotation that was previously reported in Matthews et al. (2006). We show other examples of orderly velocity fields in Figure 14. coming from dense gas peaks that are spatially separated from other structures at low emission levels. One of the examples is the dense gas surrounding the newly detected compact continuum core within Per-emb 30, while the other two have no detected protostars at their center. This highlights the ability of CLASSy data to probe the small-scale velocity structure around cores in addition to the large-scale velocity structure across the entire region.

5. The best-fit values of the centroid velocity and velocity dispersion toward the B1-c core are consistent with the results published by Matthews et al. (2006). We detect the same evidence of a rotating $\mathrm{N}_{2} \mathrm{H}^{+}$ envelope in the centroid velocity map, and detect the same increased velocity dispersion along the $\mathrm{HCO}^{+}$ and $\mathrm{HCN}$ outflow axis. Also, the $\mathrm{N}_{2} \mathrm{H}^{+}$centroid velocities and velocity dispersions across the field match well with GBT $\mathrm{NH}_{3}$ observations toward select dust cores (Rosolowsky et al. 2008a). 

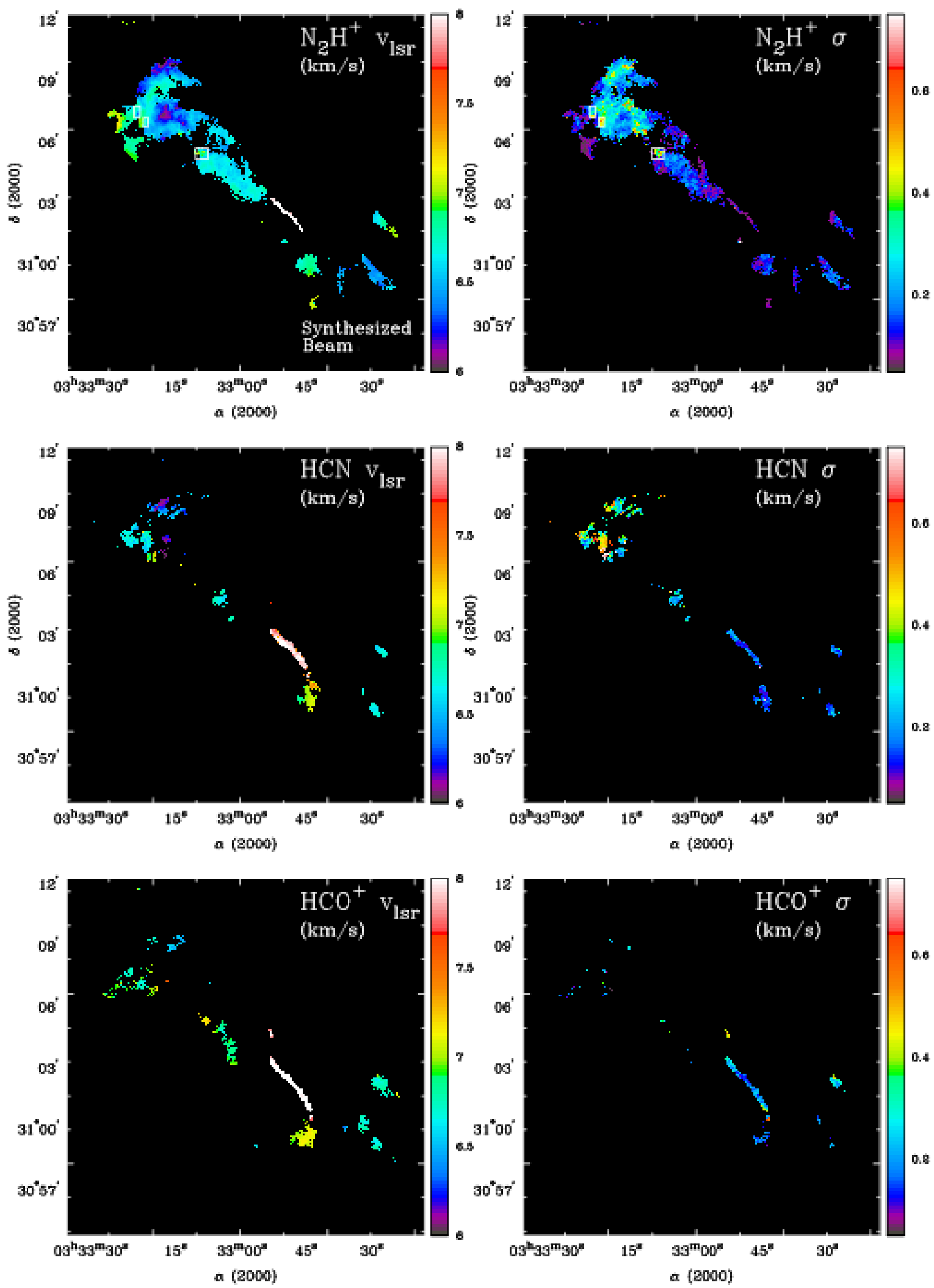

Fig. 12. - Left: Centroid velocity maps of $\mathrm{N}_{2} \mathrm{H}^{+}, \mathrm{HCN}$, and $\mathrm{HCO}^{+}(J=1 \rightarrow 0)$ emission, from top to bottom. Right: velocity dispersion maps of $\mathrm{N}_{2} \mathrm{H}^{+}, \mathrm{HCN}$, and $\mathrm{HCO}^{+}(J=1 \rightarrow 0)$ emission, from top to bottom. We masked these maps at different levels (see section 5 text) to visualize only statistically robust kinematic results. The color scales are the same across molecules. The $\sim 7^{\prime \prime}$ synthesized beam is plotted as a very small white circle in the lower right corner of the $\mathrm{N}_{2} \mathrm{H}^{+}$centroid velocity map. The small, white rectangles in the $\mathrm{N}_{2} \mathrm{H}^{+}$maps identify regions that likely contain two velocity components along the-of-sight and are excluded from analysis later in the paper. 

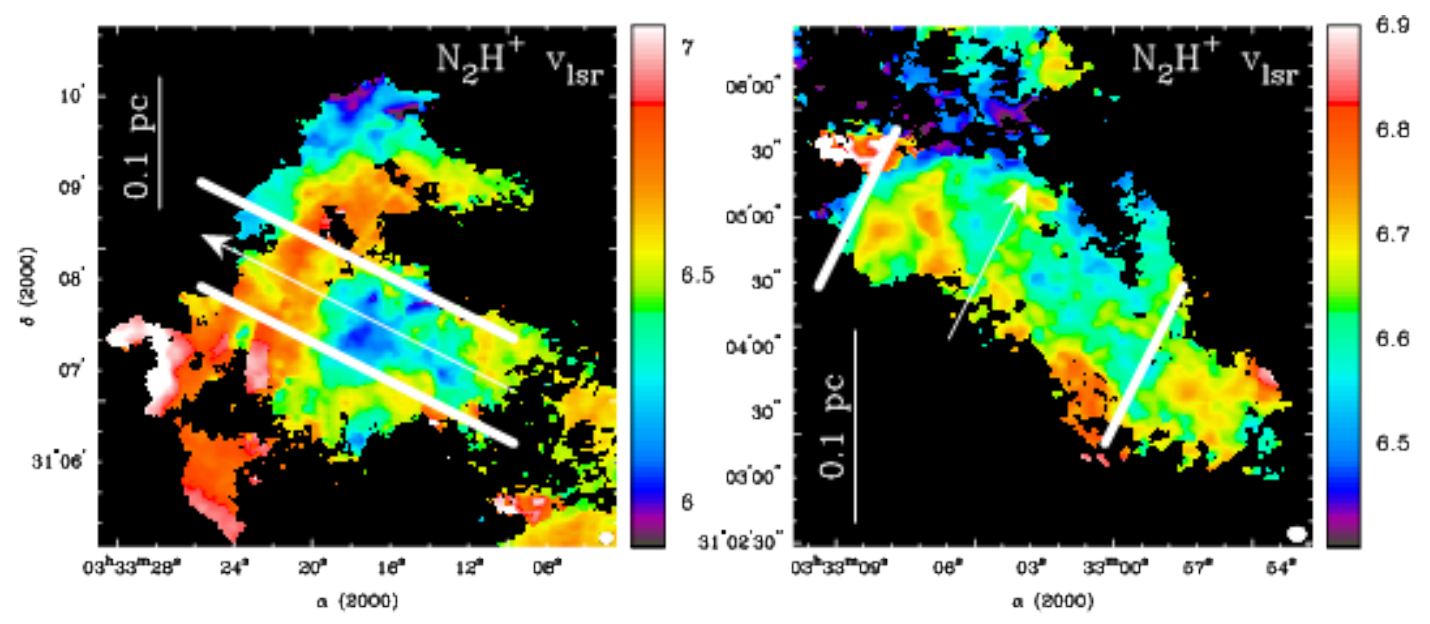

Fig. 13. - Left: Zoom-in of the main core zone centroid velocities presented in Figure 12, showing the complex velocity structure seen across this large area. The two solid lines enclose a region where we calculated the velocity gradient along the direction indicated by the arrow; we found a peak velocity gradient $\sim 10 \mathrm{~km} \mathrm{~s}^{-1} \mathrm{pc}^{-1}$ along this direction. Right: Zoom-in of the ridge zone centroid velocities. Between the solid white bars, we measured an average velocity difference $\sim 0.3 \mathrm{~km} \mathrm{~s}^{-1}$ perpendicular to the major axis of the filament. A $0.1 \mathrm{pc}$ scale bar and synthesized beam is shown in the bottom right corner of each image.
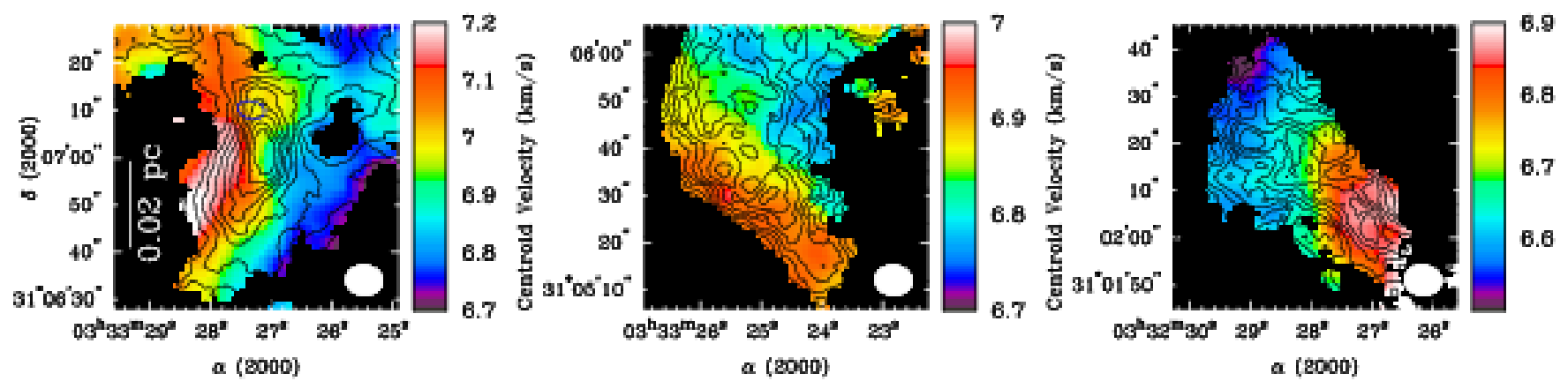

Fig. 14.- Zoom-in on three small-scale regions with orderly centroid velocity fields, highlighting CLASSy's ability to resolve small-scale kinematic features, in addition to the large-scale structures seen in Figures 12 and 13 . The greyscale intensity is centroid velocity, and the contours are integrated intensity (contour levels in intervals of $10 \%$ of the image peak intensity). The left image shows the dense gas around the compact continuum core toward Per-emb 30 (the continuum core is identified with a single contour; colored blue in the online version). The center image is of gas south of Per-emb 30 in the main core zone, and the right image is of gas in the SW clumps zone. The synthesized beam is shown in the lower-right corner of each image, and a $0.02 \mathrm{pc}$ scale bar is shown in the left image.

\section{DENDROGRAM ANALYSIS OF $\mathbf{N}_{2} \mathbf{H}^{+}$}

The previous two sections highlighted the dense gas morphology and kinematics across Barnard 1. Here we quantitatively analyze the $\mathrm{N}_{2} \mathrm{H}^{+}$position-position-velocity cube to create a census of dense gas structures. We chose $\mathrm{N}_{2} \mathrm{H}^{+}$over $\mathrm{HCN}$ and $\mathrm{HCO}^{+}$because it is our best tracer of the dense gas that is actively participating in star formation - it closely mimics the dust emission and is less affected by absorption from lower density foreground gas than $\mathrm{HCN}$ and $\mathrm{HCO}^{+}$.

Several methods exist for identifying structures in an image or cube, and the most appropriate method depends on the data and science goals. In Appendix A, we compare a widely used clump-finding algorithm 
to the dendrogram method, and conclude that a dendrogram analysis is more suitable for identifying resolved, dense gas structures in nearby molecular clouds. A dendrogram tracks emission structure as a function of isocontour level intensity, and represents the structure as a tree hierarchy made up of leaves and branches (Houlahan \& Scalo 1992; Rosolowsky et al. 2008b; Goodman et al. 2009). Leaves are smaller-scale, brighter objects at the top of the emission hierarchy that do not break-up into further substructure, while branches are the larger-scale, fainter objects lower in the hierarchy that do break-up into substructure. The major benefit of a dendrogram analysis over a clump-finding analysis rests in this ability to represent all of the spatial scales in a dataset, as opposed to forcing all emission into distinct clumps associated with a local peak. See Appendix A and Goodman et al. (2009) for more discussion.

In Appendix B, we describe our modifications to the standard dendrogram algorithm that enable non-binary hierarchies, and we argue that non-binary dendrograms provide a more statistically meaningful way to represent hierarchical emission in the presence of noise. The important modifications include: 1) restricting branching to intensity steps equal to integer values of the 1- $\sigma$ sensitivity of the data instead of allowing branching at infinitely small intensity steps, and 2) using an algorithm that can cluster more than two objects into a single group instead of being restricted to clustering two objects at a time. These changes create an observable emission hierarchy within the noise limits of the data, and allow the quantification of the hierarchical complexity of a dendrogram using tree statistics. The rest of this section focuses on our non-binary dendrogram analysis of the $\mathrm{N}_{2} \mathrm{H}^{+}$gas in Barnard 1 .

\subsection{The Non-binary Dendrogram}

We used our new non-binary dendrogram code to identify gas structures traced by the isolated hyperfine component of $\mathrm{N}_{2} \mathrm{H}^{+}$. We chose to use the isolated hyperfine component of $\mathrm{N}_{2} \mathrm{H}^{+}$since it is sufficiently separated from other components in velocity space to prevent contaminating our object identification along the velocity axis. Before running the data through the dendrogram code, we binned the cube by two velocity channels to improve the signal-to-noise. No velocity information was lost since there are no lines of sight that contain two or more independent structures within $1.0 \mathrm{~km} \mathrm{~s}^{-1}$ of each other. We found that binning by two channels provides the most improvement to signal-to-noise without biasing the maps towards wide-line regions. The 1- $\sigma$ sensitivity of the binned data cube is $0.094 \mathrm{Jy}_{\text {beam }}{ }^{-1}$.

Our non-binary dendrogram code takes the same input parameters as the standard IDL implementation discussed in Rosolowsky et al. (2008b). We ran it with the following critical inputs and parameters: (1) a masked cube containing all pixels greater than or equal to 4- $\sigma$ intensity, along with adjacent pixels of at least 2.5- $\sigma$ intensity, (2) a set of local maxima greater than or equal to all their neighbors in $10^{\prime \prime}$ by $10^{\prime \prime}$ by three channel $\left(0.94 \mathrm{~km} \mathrm{~s}^{-1}\right)$ spatial-velocity pixels, (3) a requirement that a local maximum must peak $2-\sigma$ above the intensity where it first merges with another local maximum for it to be considered a leaf, (4) a requirement of at least three synthesized beams of spatial-velocity pixels for a leaf to be considered real.

The non-binary dendrogram shown in Figure 15 contains 41 leaves and 13 branches. The vertical axis of the dendrogram represents the intensity range of the pixels belonging to a leaf or branch. The horizontal axis does not normally carry physical meaning, but in this case we arranged the leaves and branches according to zone. Figure 16 shows two-dimensional representations of all leaves overlaid on the $\mathrm{N}_{2} \mathrm{H}^{+}$integrated intensity map; these representations were created by integrating over the velocity axis of the leaves and contouring the maximal RA-DEC extent of the integrated emission. The leaves that peak at least $6-\sigma$ in intensity above their first branch are colored green (leaves 10, 25, and 39). Leaf 25 is the strongest, with a peak intensity of

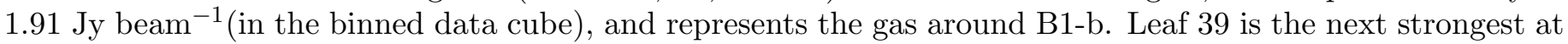

$1.77 \mathrm{Jy}_{\text {beam }}{ }^{-1}$, and represents gas around B1-c. Leaf 10 peaks at $1.04 \mathrm{Jy} \mathrm{beam}^{-1}$, and represents gas around the newly detected compact continuum source within Per-emb 30. As a reminder that the identification of leaves and branches was done in three dimensions, Figure 17 shows five $\mathrm{N}_{2} \mathrm{H}^{+}$velocity channels near the B1-b 
cores with the isocontours of leaves 24,25 , and 40 , and branch 41, identified with distinct colors.

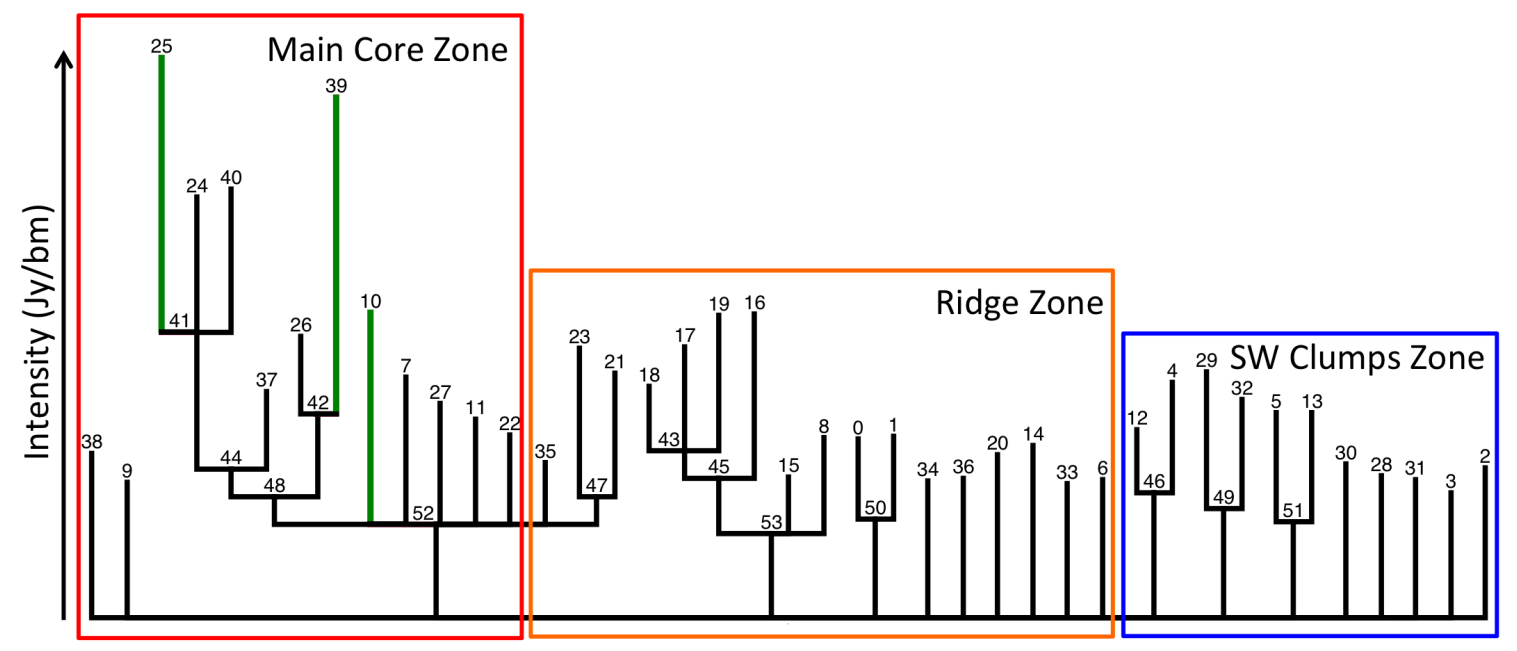

Fig. 15. - The non-binary dendrogram for Barnard 1 is shown; it represents the hierarchical structure of the $\mathrm{N}_{2} \mathrm{H}^{+}$ gas. There are 41 leaves (numbered 0 through 40), and 13 branches (numbered 41 through 53) on the dendrogram. The vertical axis represents the $\mathrm{Jy}_{\text {beam }}{ }^{-1}$ intensity for a given location within the gas hierarchy. Branching occurs in integer multiples of the 1- $\sigma$ sensitivity of the binned data cube used in this analysis. The horizontal axis is ordered according to zone, though the order within a given zone has no physical meaning. Leaves 10, 25, and 39 (colored green in the online version) peak at least 6- $\sigma$ above their first merge level.

We are using the 6- $\sigma$ contrast criteria to highlight the strongest leaves before we are able to confidently discuss the virial boundedness of actual "cores" and "clumps" - defining bound cores requires accurate measurements of mass to go along with our high-resolution kinematic information, which is beyond the scope of this paper. We chose a 6- $\sigma$ contrast to highlight the gas in Barnard 1 that is located near existing compact continuum objects - this gas is likely bound. We will apply the same contrast cut to other CLASSy regions. While we cannot discuss boundedness of individual gas cores at this stage, we can discuss the properties of the strongest, highest-column density, dendrogram features and compare them to weaker features in the field. Leaves with 6- $\sigma$ or greater contrast will be referred to as high-contrast leaves later in the paper, and the rest of the leaves will be called low-contrast leaves. 


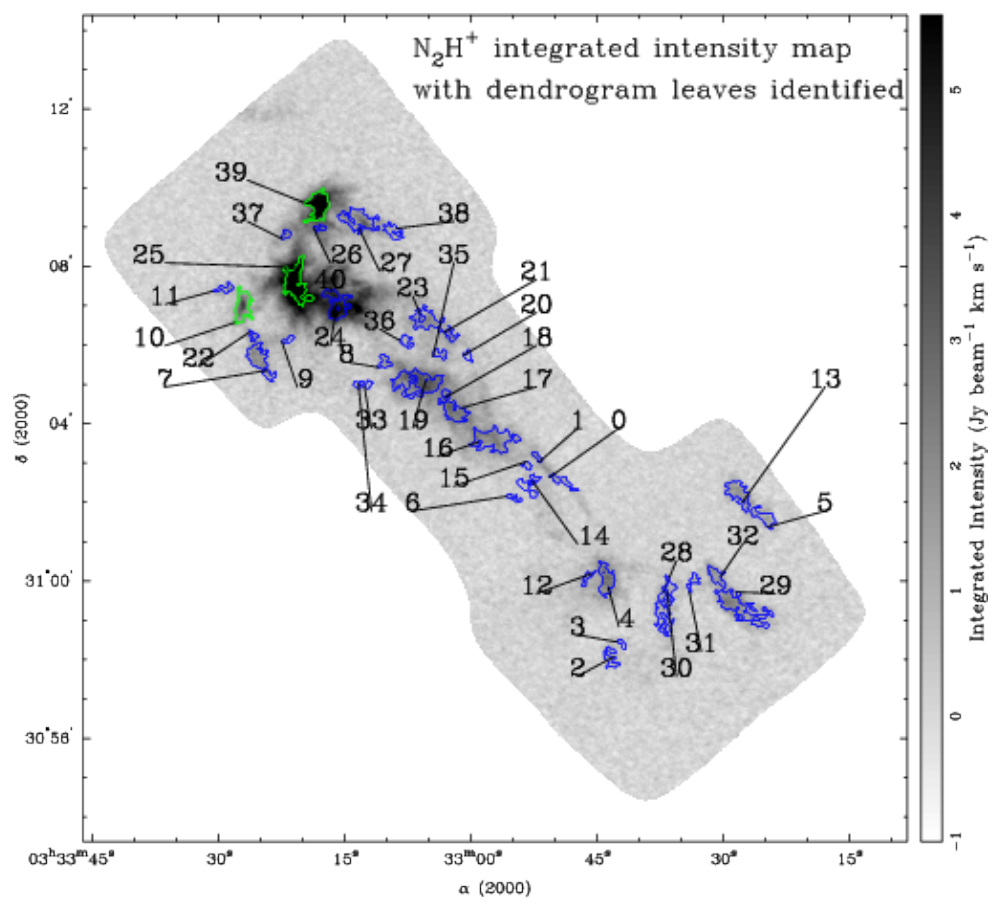

Fig. 16. - The $\mathrm{N}_{2} \mathrm{H}^{+}$integrated intensity map $\left(\mathrm{Jy} \mathrm{beam}^{-1} \mathrm{~km} \mathrm{~s}^{-1}\right)$ is shown in greyscale. The overplotted contours (green and blue in the online version) are two-dimensional representations of the three-dimensional (PPV) leaf structures found in Barnard 1. Green contours represent leaves with contrast greater than 6- $\sigma$. Each leaf is labeled with its number that can be referenced to Figure 15 and Table 5 . 


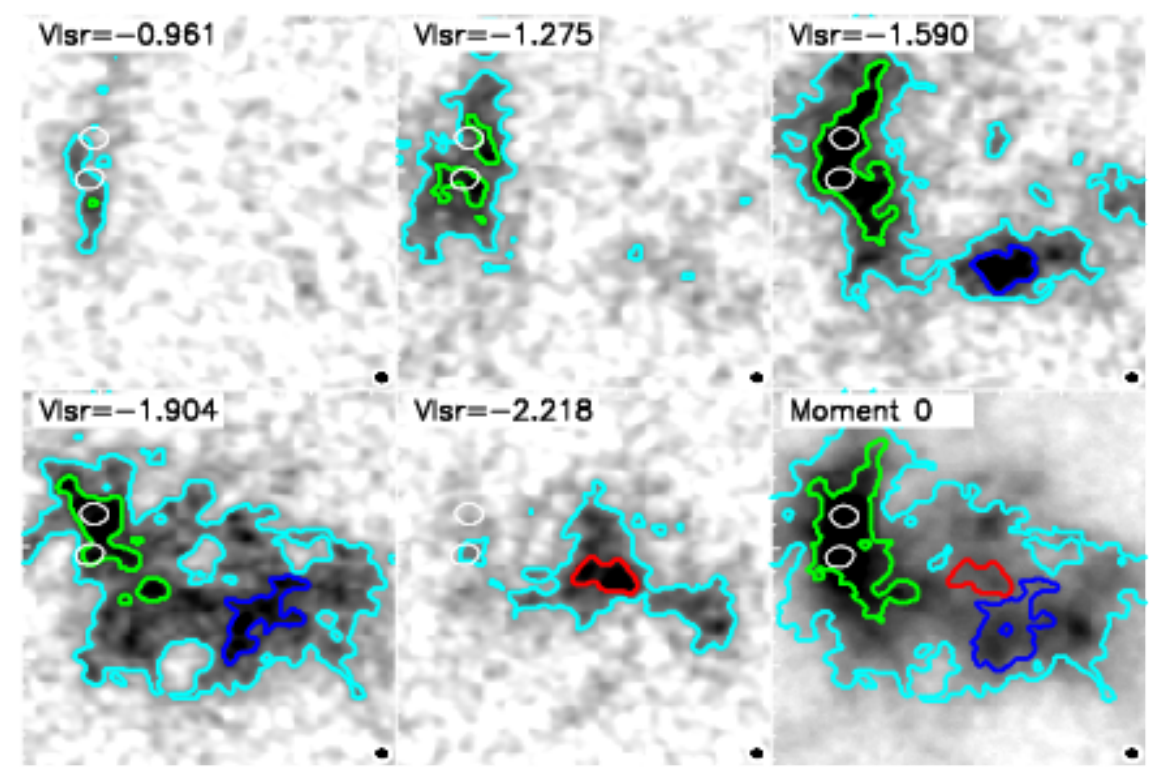

Fig. 17.- $\mathrm{N}_{2} \mathrm{H}^{+}$emission surrounding the B1-b double core, viewed across five binned velocity channels used in the dendrogram analysis, and across the moment zero map. For the channel maps, the greyscale represents the intensity of emission $\left(\mathrm{Jy} \mathrm{beam}^{-1}\right.$ ), and the colored contours (individually colored in the online version) represent the spatial extent of the dendrogram leaves and branches in that given channel. The single white contours in all maps represent the 4- $\sigma$ detection level of the B1-b continuum sources. In the online version, leaf 25 is green, 40 is red, and 24 is blue, while branch 41 is cyan. For the moment zero map, the greyscale represents the integrated intensity of emission (Jy beam ${ }^{-1} \mathrm{~km} \mathrm{~s}^{-1}$ ), and the colored contours represent two-dimensional representations of the three-dimensional dendrogram structures seen in the channel maps.

The tree structure presented here captures the qualitative hierarchical nature of the dense gas in Barnard 1 , but it does not give quantitative information about the hierarchy or physical properties of the leaves and branches. Doing science with a dendrogram requires extracting tree statistics and physical properties of the leaves and branches, such as size, axis ratio, linewidth, mass, luminosity, etc. We present three simple tree statistics and the spatial and kinematic properties of leaves and branches in this paper. In future papers, we will perform more detailed comparisons between dendrogram properties across multiple CLASSy regions.

\subsection{Dendrogram Tree Statistics}

Computation of tree statistics from a non-binary dendrogram is one method of quantifying a tree structure. Houlahan \& Scalo (1992) were the first to develop tree statistics for the astronomy community in order to quantify the hierarchical nature of molecular cloud structure (see their discussion of "merged trees," which are analogous to our non-binary trees). They describe several statistics that can be computed from non-binary trees; we describe three of the simplest statistics here.

The maximum branching level is the integer number of branching steps needed to reach the leaves at the top of the hierarchy. A region that has undergone a lot of hierarchical fragmentation will have more levels than a region that has not fragmented, or that has fragmented in a single step into several pieces with similar properties. Branching levels are defined upward from the base of the tree; the base $\left(0 \mathrm{Jy} \mathrm{beam}^{-1}\right.$ intensity) is considered level 0. An isolated leaf that sprouts directly from the base is at level 0 , while a leaf that grows from a branch one level above the base is at level 1. The maximum branching level in our Barnard $1 \mathrm{~N}_{2} \mathrm{H}^{+}$ dendrogram is level 4, where leaves 24, 25, and 40 merge together into branch 41 (see Figure 15). These leaves 
are in the main core zone, which is the only zone with detections of $3 \mathrm{~mm}$ compact continuum cores and Class 0/I YSOs. It is reasonable that the largest amount of fragmentation has occurred in the region with the most young star formation activity. That being said, the ridge zone and SW clumps zone have maximum branching levels of three and two, respectively, so we are dealing with small variations of this statistic within Barnard 1.

A related tree statistic is the mean path length of all segments in a dendrogram. The path length of a segment is defined as the number of branching steps required to go from a leaf to the bottom of the tree (there are as many path lengths in a dendrogram as there are leaves). For example, the path length of leaf 25 is four, while the path length of leaf 37 is three - the path length of leaf 30 is zero because it directly sprouts from the bottom of the tree. The mean path length in the whole Barnard $1 \mathrm{~N}_{2} \mathrm{H}^{+}$dendrogram is 1.3 levels, while it is 2.4, 1.2, and 0.5 for the main core zone, ridge zone, and SW clumps zone, respectively. Again, it is logical that the main core zone, which is actively fragmenting into young stars, has the largest mean path length.

It is important to point out that these statistics would be different if we used the standard dendrogram algorithm instead of our non-binary one. The standard algorithm forces binary branching by introducing extra branching steps to ensure that only two objects merge at a time. For example, leaves 24, 25, and 40 would not all be allowed to merge into branch 41 even though that is what the data suggests is happening-leaves 24 and 25 would be merged into one branch, which would then be merged with leaf 40 into a second branch. This inflates the path length of leaves 24 and 25, and increases the maximum branching level of the tree. See Appendix B for more details about differences between the standard dendrogram algorithm and the new non-binary algorithm.

The third simple tree statistic is the mean branching ratio of all branches in a tree. The branching ratio for a single branch is defined as the number of substructures into which it fragments directly above it in the hierarchy. For example, the branching ratio of branch 41 is three, because it has three leaves directly above it. A very hierarchical region, where every object fragments into two nested objects, will have a mean branching ratio of 2 , while a region that has fragmented in a single step into several pieces with similar properties will have a much larger mean branching ratio. The mean branching ratio in our Barnard $1 \mathrm{~N}_{2} \mathrm{H}^{+}$dendrogram is 3.9. We include the branching ratio for the base of the tree in this calculation, even though it is not explicitly defined with a branch number - this ensures that leaves that sprout directly from the base are factored in. (Note that the branching ratio above the tree base is always fixed at 2 for the standard dendrogram algorithm that forces binary branching.)

These simple statistics provide measures of the amount and type of fragmentation a given region has undergone; a region with a lot of hierarchical fragmentation will have more levels, a larger mean path length, and a smaller branching ratio, compared to a region that is just beginning to form strong overdensities. A caveat is that the absolute values of the branch statistics depend on algorithm parameters and choice of allowed branching steps (reminder that we restrict branching to intensity steps equal to integer values of the 1- $\sigma$ sensitivity of the data instead of allowing branching at infinitely small intensity steps). However, the statistics can be compared across trees as a differential measurement if the same algorithm parameters and allowed branching steps are used; as pointed out by Houlahan \& Scalo (1992), the power of tree statistics is strongest when comparing regions with different star formation properties. We will compare the Barnard 1 tree statistics with the tree statistics of NGC 1333 and L1451 in an upcoming paper to explore whether the hierarchical complexity of dense gas represented by tree statistics correlates with the diversity of star formation activity in the western half of Perseus.

Another caveat arises from linking dendrogram hierarchical complexity to cloud fragmentation - tree statistics can be affected by projection effects. All observations suffer from projection effects when transforming the true position-position-position information of a molecular cloud to observable position-position-velocity information (for an in-depth discussion of projection effects in spectral line data, see Beaumont et al. 2013). Projection effects distort optically thick, space-filling emission more than optically thin emission concentrated in one part of the cloud. These effects are not overly concerning in our data because the isolated hyperfine 
component of $\mathrm{N}_{2} \mathrm{H}^{+}$is optically thin, and the $\mathrm{N}_{2} \mathrm{H}^{+}$emission is arising only from the densest parts of the cloud. If our data does suffer from projection effects, we assume that our large mapping areas create a big enough sample of dendrogram structures so that equal numbers of projection effects occur in each region we will compare.

\subsection{Dendrogram Spatial Properties}

The size of an object is one of its most fundamental properties, but defining the sizes of irregularly shaped objects in a uniform way is not trivial. This is one challenge of high-angular-resolution surveys of nearby molecular clouds - CLASSy resolves a rich morphology of structures with a range of irregular shapes.

When fitting for the size of a leaf or branch, we consider all pixels in its plane-of-sky footprint (as opposed to only considering the "onion-layer" emission of each individual branch, which is used to derive kinematic properties in the next section-see Figures 18 and 19 for examples). We use the regionprops program in MATLAB to fit an ellipse to the integrated intensity footprint of each dendrogram object. All pixels are given equal weight so that the ellipse is preferentially fit to the largest scale of the object - this prevents strong emission at the center of an object from driving the fit towards a smaller scale. The fit is defined with an RA centroid, DEC centroid, major axis, minor axis, and position angle. The location, major axis, minor axis, and position angle are directly determined by the fit, and are listed in columns 2-6 in Table 5. We do not report formal uncertainties on these values since the spatial properties of irregularly shaped objects are dependent on the chosen method, and we do not want to mislead the reader as to the "certainty" of these values.

To quantify the shape of an object, we use the axis ratio and filling factor of the fit (columns 7 and 8 of Table 5). The axis ratio is defined as the ratio of the minor-to-major axis; a circular fit will have an axis ratio of one, and a highly elongated fit will have an axis ratio approaching zero. The filling factor is defined as the area of emission within the fitted ellipse divided by the area of the fitted ellipse; a circular object and an elliptical object will both have a filling factor near one, but an irregular object will likely have a filling factor much less than one, regardless of the axis ratio of the fitted ellipse. We lastly define an object size (column 9 of Table 5) as the geometric mean of the major and minor axes. The values reported in Table 5 have been converted to parsecs, assuming a distance of 235 pc. Figure 18 shows example spatial property fitting results for leaves 26 and 39 and branch 42 . 


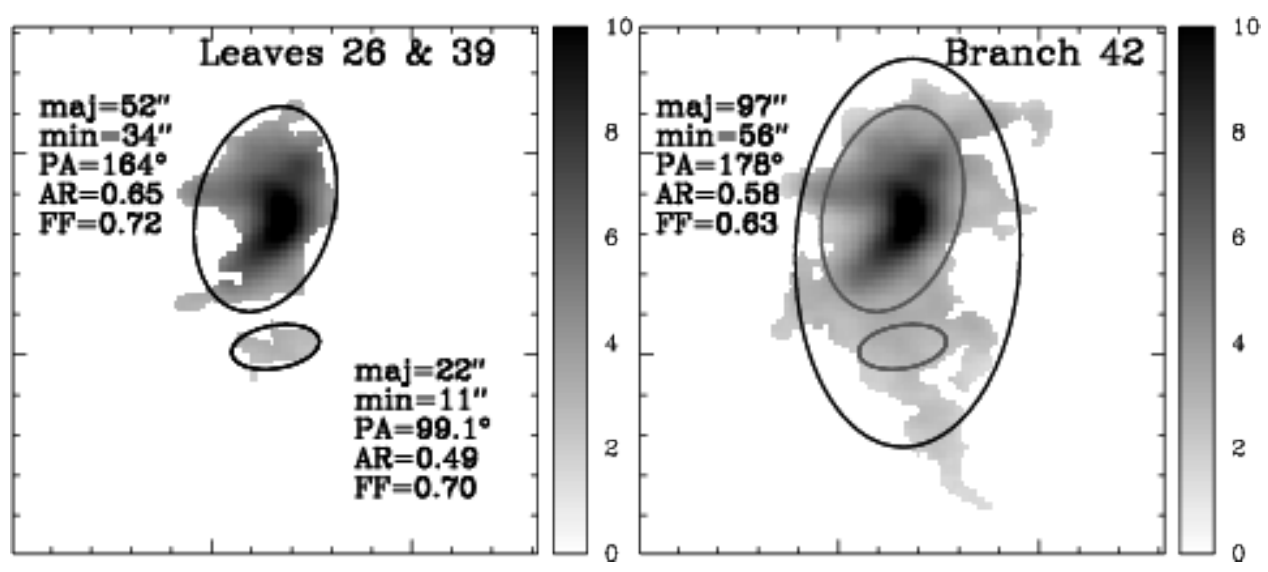

Fig. 18. - Example fits for the spatial properties of leaves and branches. The left panel shows the $\mathrm{N}_{2} \mathrm{H}^{+}$integrated intensity $\left(\mathrm{Jy} \mathrm{beam}^{-1} \mathrm{~km} \mathrm{~s}^{-1}\right.$ ) within the two-dimensional footprints of leaves 26 and 39 . We estimated the shape of these leaves with regionprops in MATLAB; the fits are shown with the solid black ellipses, and the fit parameters are listed. The axis ratio (AR) is the ratio of minor-to-major axis, and the filling factor (FF) is the number of greyscale pixels within the fitted ellipse divided by the total number of pixels within the ellipse. The right panel shows the $\mathrm{N}_{2} \mathrm{H}^{+}$ integrated intensity within the footprint of branch 42 , with its ellipse fit shown in black, and the ellipse fits of the leaves in grey. When fitting for the size of a branch, we include emission from the branch itself and all objects above it in the dendrogram - this results in a filled-in integrated intensity map for the fitting process, whereas using the emission from a single branch would give an "onion-layer"-like structure.

Histograms of the size, filling factor, and axis ratio for all leaves and branches are plotted in the top row of Figure 20. The trend in size is obvious - branches represent larger structures than the leaves that peak within them. Leaf sizes range from about $0.01 \mathrm{pc}$ to $0.07 \mathrm{pc}$, while branch sizes range from about $0.08 \mathrm{pc}$ to $0.34 \mathrm{pc}$. The high-contrast leaves are larger than the majority of low-contrast leaves. Nearly $25 \%$ of leaves have filling factors greater than 0.8 , while all branches have filling factors less than 0.8 . This shows that leaves are better fit as regular shapes compared to branches, but there is still a large population of irregularly-shaped leaves that are far from round or filamentary. The objects with the maximum axis ratios (leaf 14 and branch 46) are paired with filling factors below 0.8, indicating that they are not regularly shaped spheroids as the axis ratio alone might be interpreted. There are no obvious distinctions between high-contrast and low-contrast leaves in filling factor or axis ratio.

\subsection{Dendrogram Kinematic Properties}

One strength of CLASSy data is the kinematic information across a wide range of spatial scales. We can use the integrated intensity footprints of the dendrogram objects (leaf footprints shown in Figure 16) in combination with the centroid velocity and velocity dispersion maps in Figure 12 to determine the kinematic properties of leaves and branches. The four properties in Table 5 that we focus on are: the mean and rms centroid velocity $\left(\left\langle\mathrm{V}_{\mathrm{lsr}}\right\rangle\right.$ and $\Delta \mathrm{V}_{\mathrm{lsr}}$, respectively), and the mean and rms velocity dispersion $(\langle\sigma\rangle$ and $\Delta \sigma$, respectively).

We illustrate how we derive these properties for leaves and branches in Figure 19. For leaves, we mask the full $\mathrm{N}_{2} \mathrm{H}^{+} \mathrm{V}_{\text {lsr }}$ and $\sigma$ maps with the integrated intensity footprint of each leaf, and calculate the error weighted mean and standard deviation of the masked pixels within the masked footprints. For branches, we mask the full $\mathrm{N}_{2} \mathrm{H}^{+} \mathrm{V}_{\mathrm{lsr}}$ and $\sigma$ maps with the integrated intensity footprint of each branch, excluding pixels associated with any leaves or branches inside the branch footprint. We calculate the error weighted mean and standard deviation of the remaining pixels belonging exclusively to the branch. By excluding leaf pixels, we are calculating the kinematic properties of branches only at the larger spatial scales that are not captured by 
the leaves within them - this method allows us to parse kinematics across different spatial scales, which enables a size-linewidth analysis in the next section. We only report kinematic properties of leaves and branches in Table 5 that have three or more synthesized beams worth of kinematic pixels; a low signal-to-noise feature may be strong enough to be selected as a dendrogram object, but still too weak to have enough kinematic data points based on our line fitting criteria discussed in section 5 .
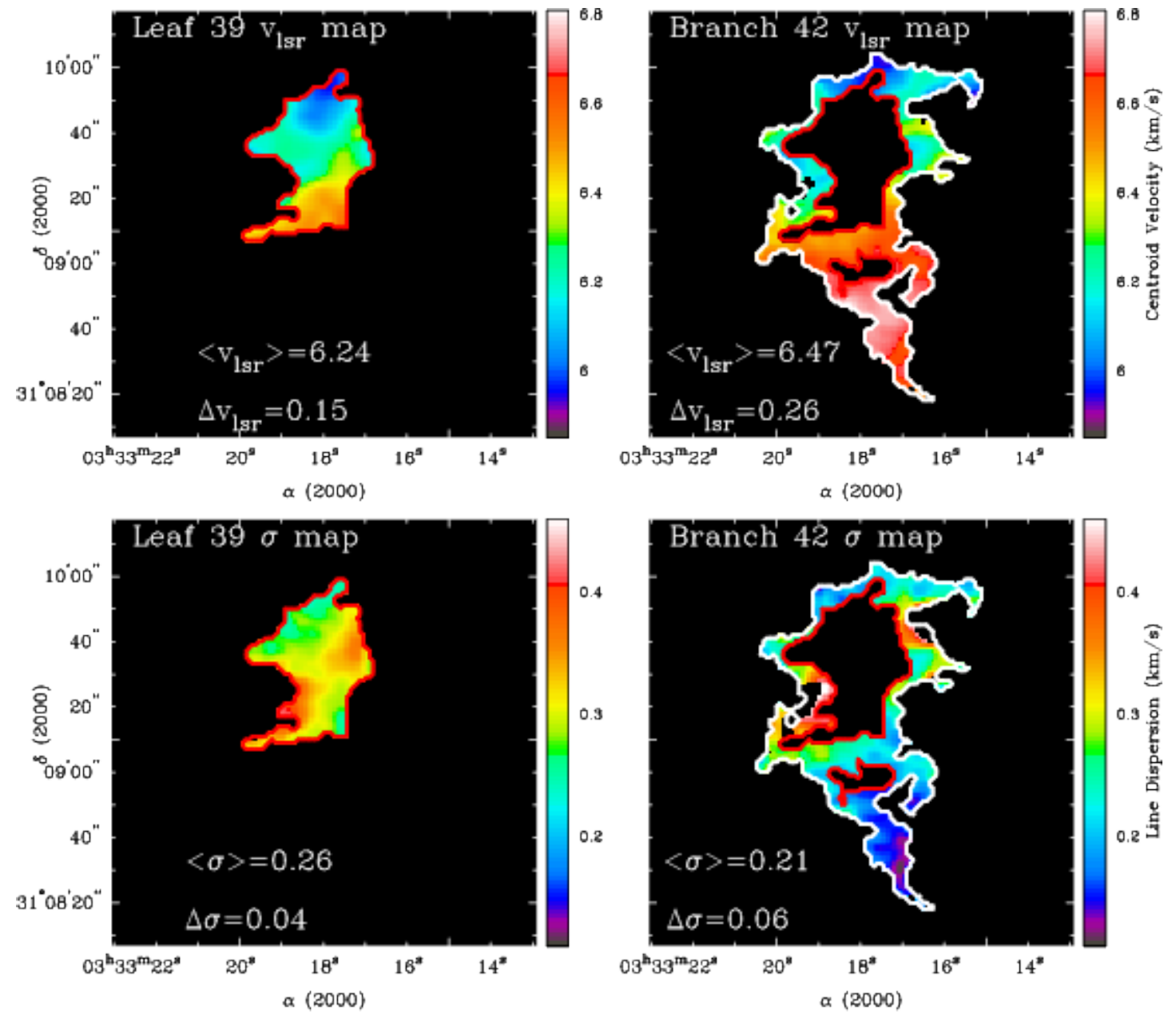

Fig. 19.- Examples of how we calculate the kinematic properties of leaves and branches, using the leaf emission towards B1-c, and the branch emission directly surrounding it. Upper left: The integrated intensity footprint of leaf 39 is represented by the contour (colored red in the online version). The best fit centroid velocities $\left(\mathrm{V}_{\mathrm{lsr}}\right)$ from Figure 12 are shown within that contour; to find the mean centroid velocity $\left(\left\langle\mathrm{V}_{\mathrm{lsr}}\right\rangle\right)$ for leaf 39 , we calculate the weighted mean of those pixels (weighing according to fit error at each pixel), and to find the rms centroid velocity $\left(\Delta \mathrm{V}_{\mathrm{lsr}}\right)$ for leaf 39 , we calculate the weighted standard deviation of those pixels. Lower left: We show the best fit velocity dispersions $(\sigma$, not FWHM) from Figure 12 within the contour, and calculate the weighted mean velocity dispersion $(\langle\sigma\rangle)$ and rms velocity dispersion $(\Delta \sigma)$ as we did for the $\mathrm{V}_{\text {lsr }}$ values in the upper left. Upper right: The integrated intensity footprint of branch 42 is represented by the outer contour (colored white in the online version), while those of leaf 39 and leaf 26 are represented by the inner contours (colored red in the online version). The best fit $\mathrm{V}_{\mathrm{lsr}}$ from Figure 12 are shown only between the outer and inner contours. We are using information only at the branch spatial scales to calculate the kinematic properties of the branch - we exclude the kinematic properties of the leaves within the branch. As for the upper left figure, to find $\left\langle\mathrm{V}_{\text {lsr }}\right\rangle$ for branch 42 , we calculate the weighted mean of the shown pixels, and to find $\Delta \mathrm{V}_{\text {lsr }}$, we calculate the weighted standard deviation of the shown pixels. Lower right: The best fit $\sigma$ for branch 42 are shown between the outer and inner contours. 
Histograms of $\left\langle\mathrm{V}_{\mathrm{lsr}}\right\rangle, \Delta \mathrm{V}_{\mathrm{lsr}}$, and $\langle\sigma\rangle$ are plotted in the bottom row of Figure 20. The distribution of $\left\langle\mathrm{V}_{\mathrm{lsr}}\right\rangle$ shows that most of the gas in Barnard 1 is near $6.5 \mathrm{~km} \mathrm{~s}^{-1}$, while the gas from leaves 0 and 1 and branch 50 (representing the narrow filament in the ridge zone) is strongly redshifted.

There is a clear offset in the distribution of $\Delta V_{\text {lsr }}$ between leaves and branches: the majority of branches, which represent the largest objects, have larger $\Delta V_{\text {lsr }}$ than the smaller leaves. There are two effects that are likely causing this trend. The first effect is from organized velocity field motions (e.g., indicative of rotation or feedback from outflows) that exist towards a leaf and its surrounding branch. For example, the $\mathrm{V}_{\text {lsr }}$ field for the B1-c leaf and parent branch can be seen in Figure 19. The branch that directly surrounds B1-c has a larger $\Delta \mathrm{V}_{\text {lsr }}$ compared to the B1-c leaf due to larger differences between the redshifted and blueshifted velocities in the rotating branch. However, the majority of leaf-branch pairs do not exhibit matching, well-ordered velocity fields. Therefore, turbulence is the second likely explanation for this trend. Turbulence has the property of causing scale-dependent spatial correlations within a velocity field-points separated by larger distances will have larger rms velocity differences between them than will points that are closer together (McKee \& Ostriker $2007)$, meaning that branches will have larger $\Delta \mathrm{V}_{\text {lsr }}$ values than leaves.

The high-contrast leaves are preferentially shifted to larger $\Delta \mathrm{V}_{\text {lsr }}$ relative to the low-contrast leaves. The high-contrast leaves all have known protostellar objects within them, so their gas motions are most likely dominated by infall, rotation, or feedback from outflows, all of which increase the variation in the velocity field. The low-contrast leaves with the largest $\Delta \mathrm{V}_{\text {lsr }}$ tend to have more organized centroid velocity patterns (manifested as gradients) than the low-contrast leaves with the smallest $\Delta \mathrm{V}_{\mathrm{lsr}}$, indicating that they may represent gas around pre-stellar cores that are more likely to form stars in the future.

The distribution of $\langle\sigma\rangle$ differs from the distribution of $\Delta \mathrm{V}_{\mathrm{lsr}}$. The high-contrast leaves representing the gas around B1-b and B1-c have the largest $\langle\sigma\rangle$ in the entire field, and the majority of branches have $\langle\sigma\rangle$ that are similar to those of the majority of leaves. We would naively expect that if the branches represent the largest objects projected on the plane of the sky, then they should also represent the most extended objects along our lines of sight, and should therefore have the largest velocity dispersions (assuming velocity dispersion scales proportionally with length-scale, which is the case for a turbulent cloud; McKee \& Ostriker 2007). We explore these results in more detail in section 7 , where we compare the sizes of structures to their $\Delta \mathrm{V}_{\text {lsr }}$ and $\langle\sigma\rangle$ in order to probe the physical and turbulent nature of Barnard 1. 
Table 5. $\mathrm{N}_{2} \mathrm{H}^{+}$Dendrogram Leaf and Branch Properties

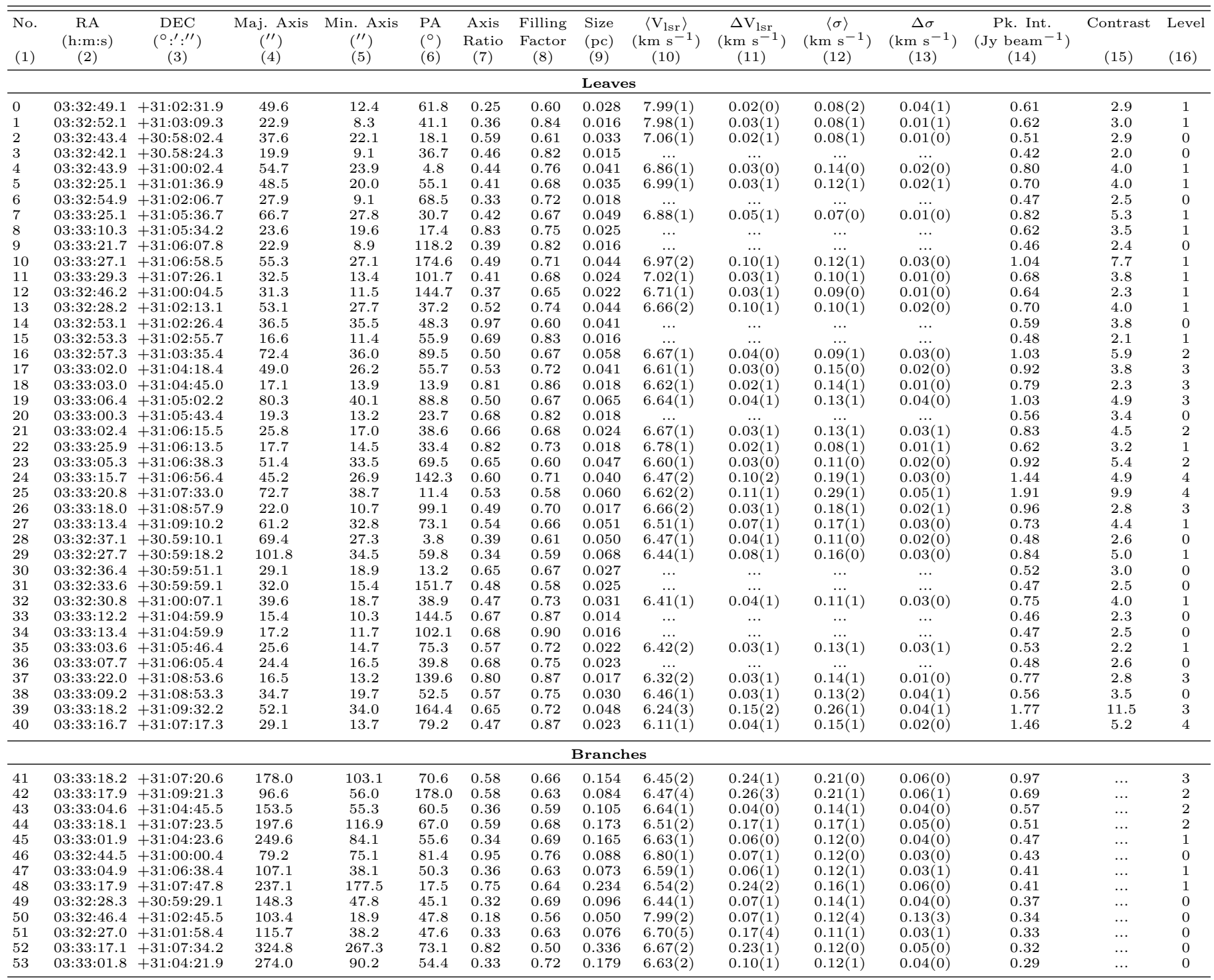

Note. - (2)-(6) The position, major axis, minor axis, and position angle were determined from regionprops in MATLAB. We do not report formal uncertainties of these values since the spatial properties of irregularly shaped objects is dependent on the chosen method.

(7) Axis ratio, defined as the ratio of the minor axis to the major axis.

(8) Filling factor, defined as the area of the leaf or branch inscribed within the fitted ellipse, divided by the area of the fitted ellipse.

(9) Size, defined as the geometric mean of the major and minor axes, for an assumed distance of $235 \mathrm{pc}$.

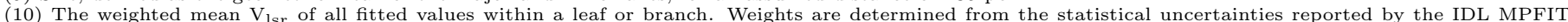

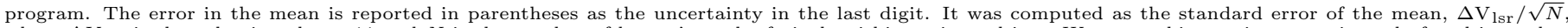
where $\Delta V_{\text {lsr }}$ is the value in column 11 and $N$ is the number of beams' worth of pixels within a given object. We report kinematic properties only for objects that have at least three synthesized beams' worth of kinematic pixels.

(11) The weighted standard deviation of all fitted $\mathrm{V}_{\mathrm{lsr}}$ values within a leaf or branch. The error was computed as the standard error of the standard deviation, $\Delta \mathrm{V}_{\text {lsr }} / \sqrt{2(N-1)}$, assuming the sample of beams was drawn from a larger sample with a Gaussian velocity distribution.

(12) The weighted mean velocity dispersion of all fitted values within a leaf or branch. The error was computed as the standard error of the mean, $\Delta \sigma / \sqrt{N}$

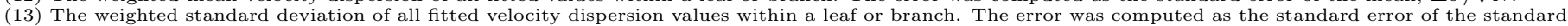
deviation, $\Delta \sigma / \sqrt{2(N-1)}$.

(14) For a leaf, this is the peak intensity measured in a single channel of our 2-channel binned dataset used in the dendrogram analysis. For a branch, this is the intensity level where the structures above it merge together.

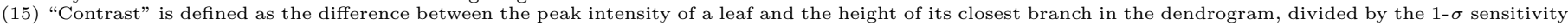
of the data.

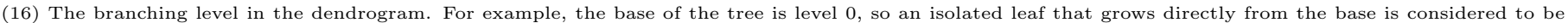
at level 0. A leaf that grows from a branch one level above the base will be at level 1, etc. 

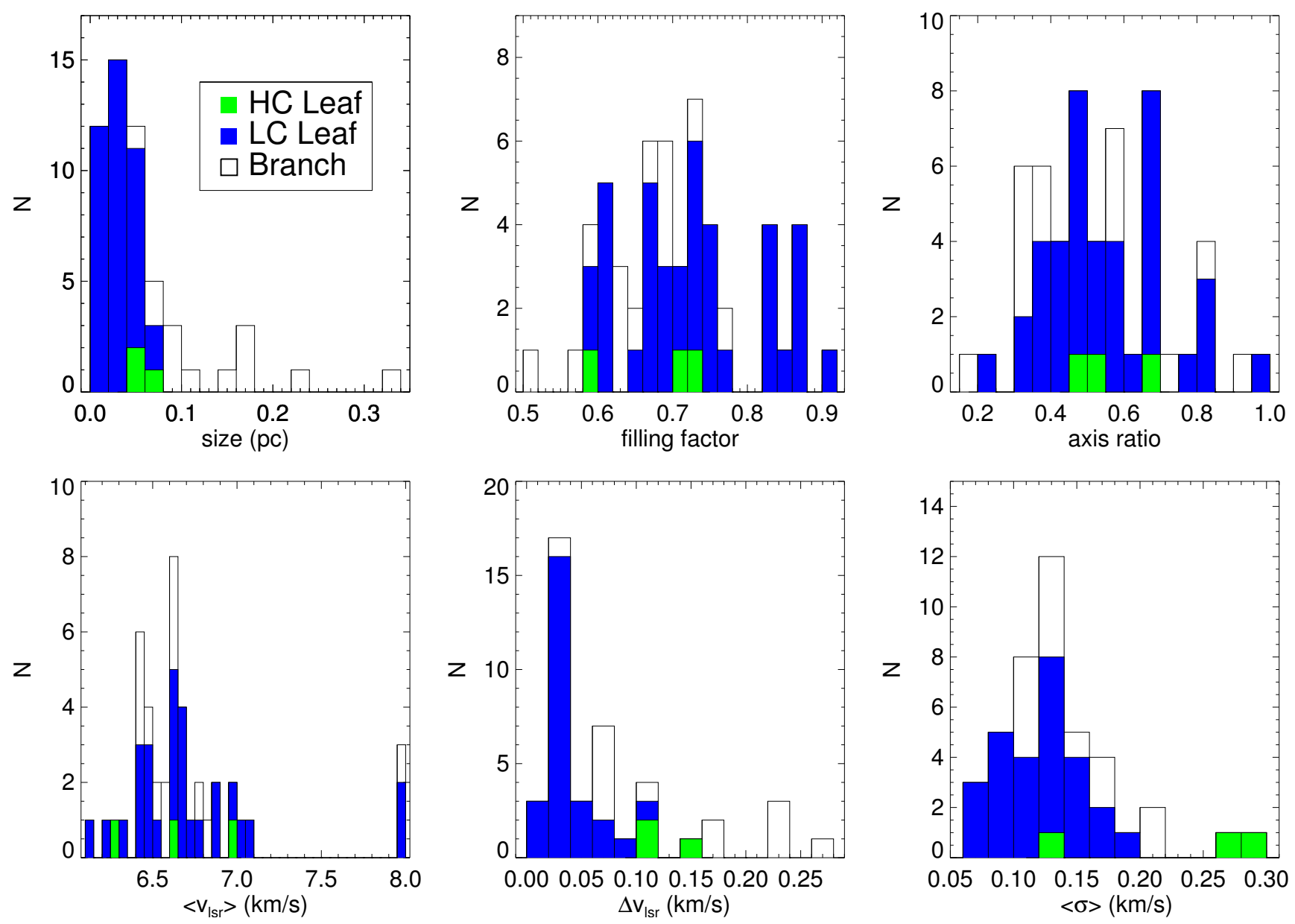

Fig. 20. - Histograms of basic dendrogram leaf and branch properties. High-contrast (HC) leaves, above 6- $\sigma$ contrast, are represented by green; low-contrast (LC) leaves, below 6- $\sigma$ contrast, are represented by blue; branches are represented by white. See the text in sections 6.3 and 6.4 for a discussion of trends seen in these histograms.

\section{A CONNECTION BETWEEN SIZE-LINEWIDTH RELATIONS AND CLOUD STRUCTURE}

This paper presented the morphology and kinematics of the dense gas in Barnard 1, along with a dendrogram analysis of the $\mathrm{N}_{2} \mathrm{H}^{+}$emission. We conclude with an analysis of the spatial and kinematic properties of dendrogram structures, and what they can tell us about the turbulent and physical nature of the Barnard 1 cloud.

A turbulent cloud will have scale-dependent spatial correlations of its velocity field that take on power law forms over a wide enough range of spatial scales (McKee \& Ostriker 2007). Size-linewidth relations are commonly used to probe turbulence; there have been numerous studies of observed and simulated molecular clouds using a wide array of observational and statistical techniques, such as correlation plots (Larson 1981; Solomon et al. 1987), structure function and autocorrelation analysis (Miesch \& Bally 1994; Ossenkopf \& Mac Low 2002), principal component analysis (Brunt \& Mac Low 2004; Heyer \& Brunt 2004), and dendrograms (Rosolowsky et al. 2008b), to name a few. The overall result is that most galactic molecular clouds exhibit power-law scaling relations consistent with turbulence in a compressible medium, where supersonic motions and 
overlapping shocks are important (so-called "Burgers turbulence"). It is well accepted from these analyses that molecular clouds are turbulent, but there are new insights that can be gained by using size-linewidth relations derived from high angular resolution observations.

\subsection{Total Linewidth of Dendrogram Structures}

Our dendrogram analysis identified $\mathrm{N}_{2} \mathrm{H}^{+}$structures in the Barnard 1 hierarchy as either leaves or branches, and we evaluated the size and kinematic properties of those objects in section 6 . Since the spatial scales of dendrogram structures in Barnard 1 range from $\sim 0.01-0.3 \mathrm{pc}$, we can utilize these structures to investigate the turbulent properties of the cloud. Before discussing size-linewidth relations that use the full angular resolution of our observations, it is useful to consider a relation that uses the "total" linewidth of each dendrogram structure - this relation can be compared with classical size-linewidth relations from Larson (1981) and Solomon et al. (1987), in which the detailed kinematic variation within individual clouds was not resolved. We calculated a total linewidth for each structure by summing all the spectra assigned to it and fitting the velocity dispersion of the resulting spectrum (the $\sigma$, not FWHM, of the line); for a visualization, see Figure 19, which shows the kinematic maps of an example leaf and branch. In that figure, all the spectra within the red contour of leaf 39 , and all the spectra within the white contours of branch 42 (including the leaf emission), are summed to calculate the total linewidths of those respective structures.

Figure 21 shows plots of projected size versus total linewidth for all dendrogram structures that have kinematic values listed in Table 5, excluding the objects associated with the peculiar narrow filament in the ridge zone (leaves 0 and 1 , branch 50 ). We separate structures in the main core zone (MCZ) from structures in the ridge and SW clumps zones (RSWZ) because the main core zone has a cluster of protostars driving outflows. Keeping the zones separate allows us to see whether or not they have different turbulent or physical properties. We also separately identify the few structures surrounding compact continuum cores, since their non-thermal gas motions are likely influenced by their central source.

The data in Figure 21 are scattered and could be fit by several functions. However, there does appear to be variation of total linewidth with size in both zones, with the linewidth varying more strongly with size in the MCZ. If we use a single power-law fit, the MCZ correlation is best fit with a slope of $0.37 \pm 0.08$, and the RSWZ correlation slope is $0.16 \pm 0.06$. The steeper slope in the MCZ could be due to outflows adding energy to the turbulence at these scales. The best-fit slope from the MCZ is consistent with the result (Larson 1981) that clouds ranging in size from 0.1 to $100 \mathrm{pc}$ have a power-law slope of 0.38 . However, we are probing the scaling relation across much smaller spatial scales than has typically been done, and unlike previous studies, we are using high-density molecular tracers that are sensitive to non-thermal motions near the sonic scale of the cloud. Therefore, directly comparing our results to studies that used larger scales and lower-density gas tracers, such as ${ }^{12} \mathrm{CO}$, should be considered with caution. 

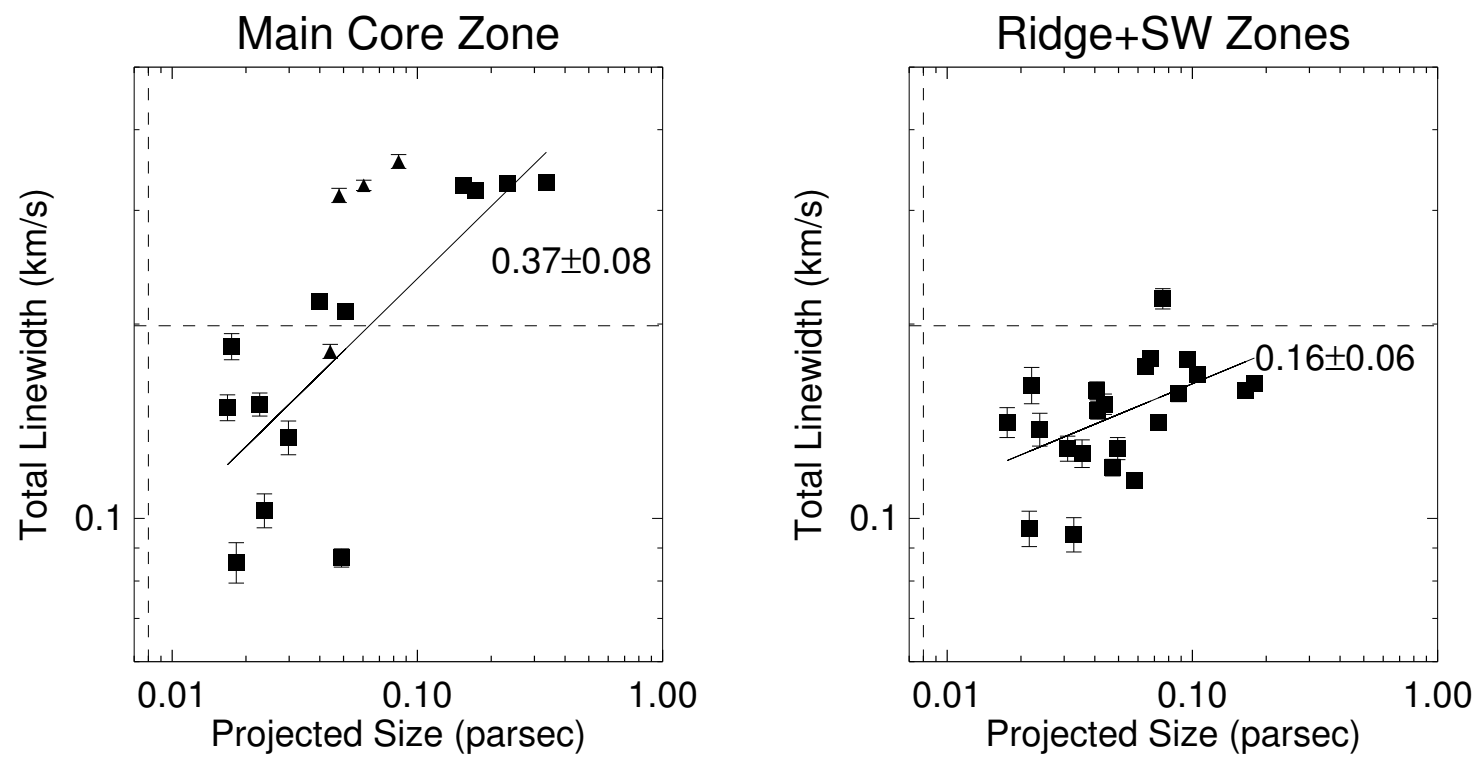

Fig. 21. - Scaling relations between projected structure size and total linewidth for the main core zone and the combined ridge and SW clumps zones. The projected size for each dendrogram structure is defined as the geometric mean of its best-fit major and minor axes. The majority of structures are labeled with squares, while the three leaves toward the three strongest compact continuum detections in main core zone are marked with triangles, along with the branch directly surrounding the B1-c leaf. The solid line represents a single power-law fit to the square points. The horizontal line represents the typical thermal speed for $\mathrm{H}_{2}$ at gas kinetic temperatures near $11 \mathrm{~K}$. The vertical line represents our spatial resolution of $\sim 0.008 \mathrm{pc}$.

\subsection{Resolved Linewidths of Dendrogram Structures}

We have two kinematic measurements of leaves and branches in Table 5 that use the full angular resolution of our dataset - they complement the total linewidth and enable a different type of size-linewidth analysis. The first measurement is the $\mathrm{V}_{\text {lsr }}$ variation, $\Delta \mathrm{V}_{\mathrm{lsr}}$. For a leaf or branch of size $\mathrm{L}, \Delta \mathrm{V}_{\mathrm{lsr}}(\mathrm{L})$ is computed by measuring the rms variation of the (beam-scale) centroid velocity over the whole structure. The second measurement is the mean non-thermal velocity dispersion, $\langle\sigma\rangle_{\text {nt }}$. To calculate $\langle\sigma\rangle_{\text {nt }}$ from $\langle\sigma\rangle$ Table 5, we remove the thermal velocity dispersion, $\sigma_{\mathrm{th}}$, of $11 \mathrm{~K} \mathrm{~N}_{2} \mathrm{H}^{+}$gas, which is the typical gas kinetic temperature across the entire Barnard 1 region (Rosolowsky et al. 2008a): $\langle\sigma\rangle_{\mathrm{nt}}=\sqrt{\langle\sigma\rangle^{2}-\sigma_{\mathrm{th}}^{2}}$. Recall that $\langle\sigma\rangle$ for a structure is defined by averaging all the individual velocity dispersions over the structure; each individual pointing captures the velocity dispersion along the line-of-sight for a given beam-width.

We compare the dependences of $\Delta \mathrm{V}_{\mathrm{lsr}}$ and $\langle\sigma\rangle_{\mathrm{nt}}$ on projected structure size in Figure 22 . Size versus $\Delta \mathrm{V}_{\mathrm{lsr}}$ for the dendrogram structures in both zones is plotted on the left; the data appears to follow a power-law relation. There is a steep correlation in both regions, with power-law slopes of $0.80 \pm 0.08$ in the MCZ and $0.69 \pm 0.17$ in the RSWZ. The value of $\Delta \mathrm{V}_{\text {lsr }}$ is very low for the smallest objects in all zones, and rises to the sound speed for the largest objects in the MCZ. Size versus $\langle\sigma\rangle_{\text {nt }}$ is plotted on the right side Figure 22 . There is a much flatter correlation between size and $\langle\sigma\rangle_{\mathrm{nt}}$ in both zones, with a power-law slope of $0.12 \pm 0.11$ in the $\mathrm{MCZ}$ and $0.04 \pm 0.07$ in the RSWZ. The mean non-thermal velocity dispersions are all sub-sonic to trans-sonic in the MCZ (though still superthermal). The two structures with largest $\langle\sigma\rangle_{\text {nt }}$ are surrounding B1-b and B1-c; it is likely that the non-thermal motions of the gas around them is boosted by rotation and/or outflows and is not purely turbulent. The $\langle\sigma\rangle_{\mathrm{nt}}$ are all sub-sonic in the RSWZ. 

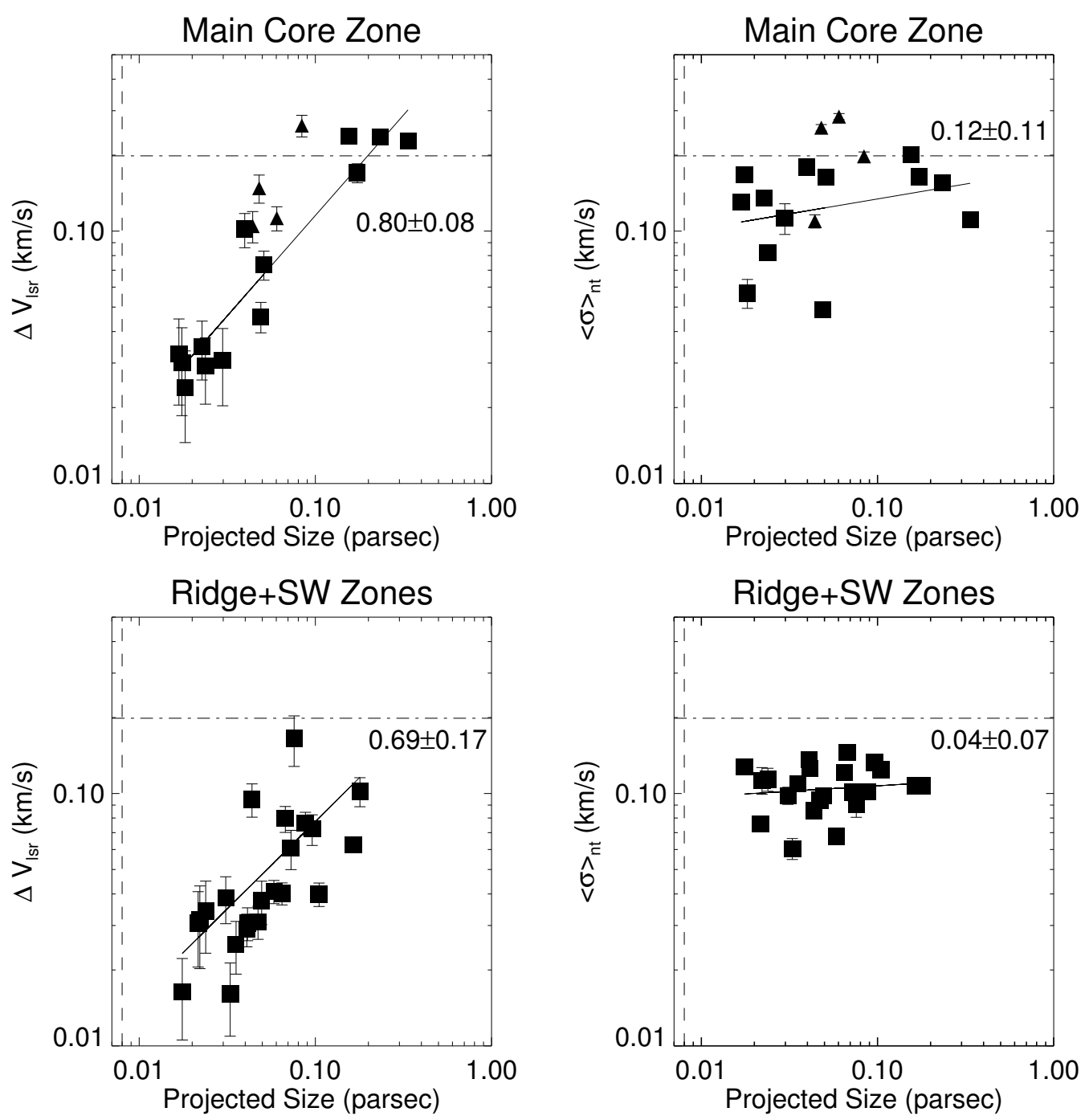

Fig. 22. - Left: Scaling relations between projected structure size and $V_{\text {lsr }}$ variation $\left(\Delta V_{\text {lsr }}\right)$ for the main core zone and the combined ridge and SW clumps zones. Right: Scaling relations between projected structure size and mean nonthermal velocity dispersion $\left(\langle\sigma\rangle_{\mathrm{nt}}\right)$ for the main core zone and the combined ridge and SW clumps zones. The solid line represents a single power-law fit to the square points. The horizontal line represents the typical thermal speed for $\mathrm{H}_{2}$ at gas kinetic temperatures near $11 \mathrm{~K}$. The vertical line represents our spatial resolution of $\sim 0.008 \mathrm{pc}$. 
Why are the dependences of $\langle\sigma\rangle_{\mathrm{nt}}$ and $\Delta \mathrm{V}_{\mathrm{lsr}}$ on projected structure size so different? In the following section, we set up a theoretical framework that can explain this disparity in terms of differences between a structure's projected size and its depth into the plane of the sky.

\subsection{Inferring Cloud Depth from Resolved Size-Linewidth Relations}

In this section, we assume that the observed non-thermal gas motions in our dendrogram structures are generated by isotropic, three-dimensional turbulence. In the previous section, we noted a few structures surrounding compact continuum cores that likely have non-thermal gas motions due to the influence of their central source; we exclude them from this analysis.

The total turbulent linewidth of a structure is most strongly influenced by the largest scale an observation encompasses, under the assumptions that the gas we observe is isotropically turbulent, and optically thin with uniform excitation conditions. If the structure is wider across the plane of the sky than it is deep into the sky, then its turbulent linewidth will be most strongly influenced by its projected size. But if the structure is deeper into the sky than in either of the projected directions, then its turbulent linewidth will be most strongly influenced by its depth.

For a structure that is many resolution elements across (the case for all of our dendrogram structures), $\Delta \mathrm{V}_{\mathrm{lsr}}$ and $\langle\sigma\rangle_{\mathrm{nt}}$ probe turbulence in different ways from the total linewidth. The value of $\mathrm{V}_{\text {lsr }}$ on any given line-of-sight is a measure of the mean motion of gas along that line-of-sight, which varies from one line-of-sight to another line-of-sight as a consequence of turbulence. The $\mathrm{V}_{\mathrm{lsr}}$ variation, $\Delta \mathrm{V}_{\mathrm{lsr}}$, is expected to increase with the projected scale of a structure if turbulence increases with scale, as is the case for the observed and simulated turbulent power spectra in molecular clouds. For any given line-of-sight, the turbulent contribution to $\langle\sigma\rangle$ will be set by the larger of the beam size and the structure depth. Since our beam size is very small $(\sim 0.008 \mathrm{pc})$, we expect that the linewidth along individual lines-of-sight will be dominated by structure depth, and thus $\langle\sigma\rangle_{\mathrm{nt}}$ for a structure will depend primarily on the mean (unseen) depth of that structure into the sky. Statistically, along many lines-of-sight for a resolved structure, the comparison of linewidths set by the projected size of the structure $\left(\Delta \mathrm{V}_{\text {lsr }}\right)$ to linewidths set by the depth of the structure $\left(\langle\sigma\rangle_{\mathrm{nt}}\right)$ should allow an estimation of the structure depth.

We can use a mathematical framework to explain how we can compare $\Delta \mathrm{V}_{\text {lsr }}$ to $\langle\sigma\rangle_{\text {nt }}$ to learn about the typical depth of gas structures. In the full three dimensional turbulence, line-of-sight turbulent velocities $\left(v_{z, \text { turb }}\right)$ can be written as

$$
v_{z, \text { turb }}(x, y, z)=\sum_{k_{x}, k_{y}, k_{z}} v_{p}\left(k_{x}, k_{y}, k_{z}\right) \exp \left[i\left(k_{x} x+k_{y} y+k_{z} z\right)\right]
$$

where $v_{p}\left(k_{x}, k_{y}, k_{z}\right)$ is the turbulent velocity power spectrum, $k_{x}=\left(2 \pi / L_{x}\right) n_{x}, k_{y}=\left(2 \pi / L_{y}\right) n_{y}$, and $k_{z}=$ $\left(2 \pi / L_{z}\right) n_{z} \cdot L_{x}$ is the size of an observed structure in the $x$ direction, and $n_{x}$ is the number of full waves of a given turbulent velocity component that fit in the $x$-direction (the same is true for the $y$ and $z$ components in these equations). If the turbulent motions are Gaussian and isotropic, then $v_{p}\left(k_{x}, k_{y}, k_{z}\right)$ is drawn from a normal distribution whose standard deviation depends only on the magnitude of the $k$-vector, $|\mathbf{k}|=\sqrt{k_{x}^{2}+k_{y}^{2}+k_{z}^{2}}$. We assign the $z$ direction as the line-of-sight, so that in Equation $4, v_{z, \text { turb }}(x, y, z)$ is the $z$-component of the turbulence, and there are other independent components in the $x$ and $y$ directions.

In this framework, the total linewidth of a structure is the sum of components with non-zero $k_{x}, k_{y}$, or $k_{z}$. The resolved linewidths $\left(\Delta \mathrm{V}_{\mathrm{lsr}}\right.$ and $\left.\langle\sigma\rangle_{\mathrm{nt}}\right)$ are explained below with the aid of Figure 23 .

The observed centroid velocity at a resolved projected position in the structure, $\mathrm{V}_{\mathrm{lsr}}(x, y)$, is the sum of components in Equation 4 that have $k_{z}=0$. The $\mathrm{V}_{\text {lsr }}$ dependence on spatial scale via $v_{p}\left(k_{x}, k_{y} ; k_{z}=0\right)$ is assumed 
to depend statistically only on $|\mathbf{k}|$ if the turbulence is isotropic. Therefore, $\mathrm{V}_{\mathrm{lsr}}(\mathrm{x}, \mathrm{y})$ has the same statistical dependence on $|\mathbf{k}|=\sqrt{k_{x}^{2}+k_{y}^{2}}$ (which we can measure) that the underlying turbulent velocity, $v_{z, \text { turb }}(x, y, z)$, has on $|\mathbf{k}|=\sqrt{k_{x}^{2}+k_{y}^{2}+k_{z}^{2}}$ (which we cannot measure). The $\mathrm{V}_{\mathrm{lsr}}(\mathrm{x}, \mathrm{y})$ variation, $\Delta \mathrm{V}_{\mathrm{lsr}}$, which is the standard deviation of $\mathrm{V}_{\text {lsr }}(x, y)$ over all resolved positions in a structure, will scale with the $(x, y)$-size of the structure according to the turbulent scaling relation of the cloud (see Dickman \& Kleiner (1985) for a discussion of spatial correlation properties of centroid velocity fields). To visualize this, we consider the structure on the left-side of Figure 23. We call the full 4-cell structure, structure A, and any contiguous 2-cell substructure within it, structure B. Structure A is wider than structure B in the $(x, y)$ directions on the sky, so the minimum, non-zero $k_{x}$ and $k_{y}$ must be smaller in structure A than structure B. Assuming $v_{p}$ has a (statistical) inverse power-law dependence on $|\mathbf{k}|$, this implies higher power in structure $\mathrm{A}$, with a greater contribution to the $\mathrm{V}_{\text {lsr }}$ variation across the $(x, y)$ face of structure A compared to structure B.

The non-thermal velocity dispersion at each resolved position in a structure, $\sigma_{\mathrm{nt}}(x, y)$, is obtained from the sum of turbulent components with all possible $k_{x}$ and $k_{y}$, and non-zero $k_{z}$, along each line-of-sight. For a given $(x, y)$, the total line-of-sight velocity is a sum: $v_{z}(z)=v_{z \text {,turb }}(z)+v_{\text {th }}(z)$, where $v_{\text {th }}$ is the thermal component of the velocity. The mean value of $v_{\mathrm{th}}(z)$ is zero, and the mean value of $v_{z, \mathrm{turb}}(z)$ is $\mathrm{V}_{\mathrm{lsr}}$. Thus, assuming that $v_{z, \operatorname{turb}}(z)$ and $v_{\text {th }}(z)$ are uncorrelated, for a given line-of-sight, the square of the total velocity dispersion, $\sigma^{2}(x, y)$, is:

$$
\sigma^{2}(x, y)=\left\langle v_{z}^{2}\right\rangle-\mathrm{V}_{\mathrm{lsr}}^{2}=\left\langle v_{z, \mathrm{turb}}^{2}\right\rangle-\mathrm{V}_{\mathrm{lsr}}^{2}+\left\langle v_{\mathrm{th}}^{2}\right\rangle+2\left\langle v_{z, \mathrm{turb}}\right\rangle\left\langle v_{\mathrm{th}}\right\rangle .
$$

The last term in Equation 5 is zero because the mean value of $v_{\text {th }}(z)$ is zero. The second-to-last term in Equation 5 is equivalent to the square of the thermal velocity dispersion, $\sigma_{\mathrm{th}}^{2} ; \sigma^{2}-\sigma_{\mathrm{th}}^{2}$ is equal to the nonthermal component of the velocity variance, $\sigma_{\mathrm{nt}}^{2}$, which means that the first two terms Equation 5 contribute to the non-thermal velocity dispersion. The combination of the first two terms is

$$
\left\langle\left|\sum_{k_{x}, k_{y} ; k_{z} \neq 0} v_{p}\left(k_{x}, k_{y}, k_{z}\right) \exp (i \mathbf{k} \cdot \mathbf{x})\right|^{2}\right\rangle
$$

where the summation includes combinations with all $k_{x}, k_{y}$, and with all $k_{z}$ except $k_{z}=0 ; \mathbf{k}$ is the wavenumber vector, and $\mathbf{x}$ is the spatial vector. This shows that the mean non-thermal velocity dispersion for our structures, $\langle\sigma\rangle_{\mathrm{nt}}$, defined as the mean of $\sigma_{\mathrm{nt}}(x, y)$ at each resolved position, will scale with the $z$-depth of the structure according to the turbulent scaling relation of the cloud. Along any given line-of-sight, a larger $L_{z}$ implies that the minimum nonzero $k_{z}=2 \pi / L_{z}$ is smaller, such that the contribution to Equation 6 would be larger (under the assumption that $v_{p}(\mathbf{k})$ statistically increases with decreasing $\left.|\mathbf{k}|\right)$. To visualize this, we can consider the structure on the right-side of Figure 23. We call the full 4-cell structure, structure A, and any contiguous 2-cell substructure within it, structure B. Structure A extends deeper into the sky than structure B, so the minimum, non-zero $k_{z}$ in structure $\mathrm{A}$ is smaller than the minimum, non-zero $k_{z}$ in structure $\mathrm{B}$. This implies higher power in structure $\mathrm{A}$, with a larger contribution to $\sigma_{\mathrm{nt}}(x, y)$ in structure A compared to structure B.

Based on the arguments above, the scaling relation between $\mathrm{V}_{\text {lsr }}$ variation and projected size, $l,\left(\Delta \mathrm{V}_{\text {lsr }}\right.$ $\left.\propto l^{q}\right)$ should have the same power-law dependence as the scaling relation between mean non-thermal velocity dispersion and depth, $d$, into the sky $\left(\langle\sigma\rangle_{\mathrm{nt}} \propto d^{q}\right)$ if the turbulence is isotropic and we are observing all the gas along each line-of-sight. But we saw in Figure 22 that the dependences of $\Delta \mathrm{V}_{\mathrm{lsr}}$ and $\sigma_{n t}$ on projected structure size are very different. The simplest explanation of this difference is that the projected size of an object need not be the same as its line-of-sight depth into the sky. The size axis in Figure 22 (right) is an estimate based on the geometric mean of the projected size. If every object, no matter its projected size, has the same depth, then we should measure a similar non-thermal velocity dispersion for those objects. Therefore, we interpret the shallow dependence of $\langle\sigma\rangle_{\mathrm{nt}}$ with size as an indication that our collection of dendrogram structures have similar depths. 

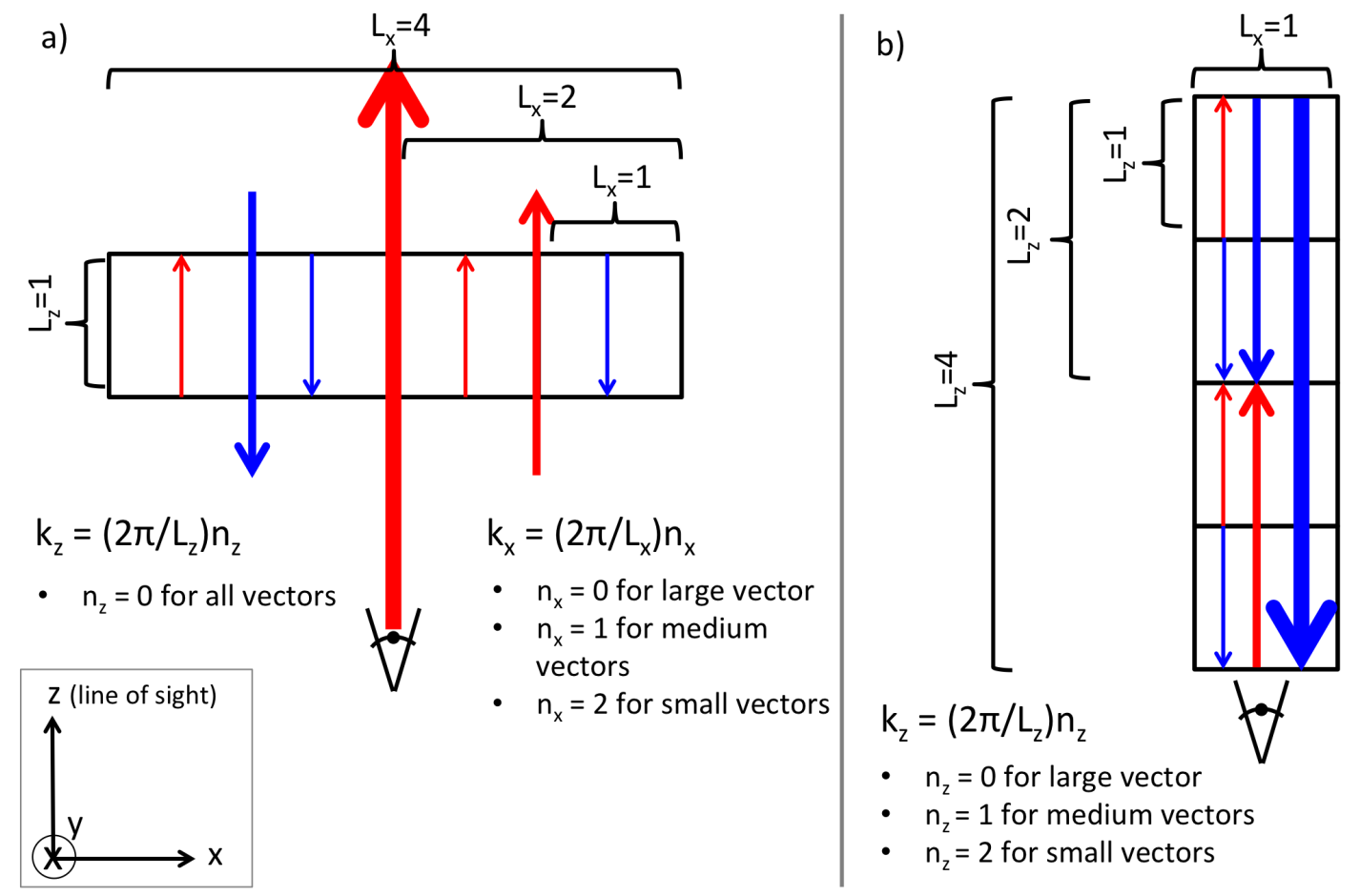

Fig. 23. - One-dimensional cartoons explaining our interpretation of the kinematics of spatially resolved dendrogram structures in the framework of isotropic turbulence. The spatial dimension of each structure is represented by four square cells, each one unit by one unit in area. The colored vectors in each figure represent turbulent velocity components along the line-of-sight (blue $=$ blue-shifted velocity component, red $=$ red-shifted velocity component). The length of the velocity vectors are not to scale, but are simply used to show that there is larger velocity power on the larger scales, and vice-versa. (a): We are looking across the resolved major axis ( $x$-direction) of this filament, and down the minor axis ( $z$-direction; line-of-sight). The $\mathrm{V}_{\mathrm{lsr}}$ of the filament at each resolved $L=1$ cell is determined by the velocity of all vectors influencing the gas in that cell with $k_{z}=0$. Since all vectors have $k_{z}=0$, for example, the left-most cell has $\mathrm{V}_{\text {lsr }}$ contributions from a small red vector, a medium blue vector, and a large red vector, while the right-most cell has $V_{\text {lsr }}$ contributions from a small blue vector, a medium red vector, and a large red vector. The $\mathrm{V}_{\text {lsr }}$ variation $\left(\Delta \mathrm{V}_{\text {lsr }}\right)$ across the four resolved cells is the standard deviation of the $\mathrm{V}_{\text {lsr }}$ in each $L=1$ cell. If this structure were half as wide, then $\Delta \mathrm{V}_{\text {lsr }}$ would decrease because each cell would only have contributions from a small and a medium vector. The non-thermal velocity dispersion along each resolved line-of-sight $\left(\sigma_{\mathrm{nt}}\right)$ is zero because there are no $k_{z} \neq 0$ vectors along the line of sight of each cell. The total velocity dispersion is a combination of vector components with $k \neq 0$, which is determined by small and medium vectors across $x$-direction. $(b)$ : We are looking down the major axis of this filament $(z$-direction; line-of-sight), through the single resolved spatial element in the $x$-direction. The $\mathrm{V}_{\text {lsr }}$ of the filament is determined by the velocity of the large vector because it is the only vector with $k_{z}=0$; oscillations of the small and medium vectors average each other out along the line-of-sight, and they do not contribute to the $\mathrm{V}_{\mathrm{lsr}}$. The $\mathrm{V}_{\text {lsr }}$ variation $\left(\Delta \mathrm{V}_{\mathrm{lsr}}\right)$ is zero because there is only one resolved $V_{\text {lsr }}$ value for this object. The non-thermal velocity dispersion $\left(\sigma_{\mathrm{nt}}\right)$ along the single line-of-sight is determined by the small and medium vectors, since both have $k_{z} \neq 0$. If this structure were half as deep, then $\sigma_{\mathrm{nt}}$ would decrease because it would be determined by the small vectors alone. The total velocity dispersion is a combination of vector components with $k \neq 0$, which is determined by small and medium vectors along the $z$-direction in this case; it equals $\sigma_{\text {nt }}$ since there is only one resolved line-of-sight. 
To illustrate these effects, we created a numerical realization of a three-dimensional, isotropic turbulent power spectrum, with power spectrum scaling $v^{2} \propto k^{-4}$. Using an arbitrary length scale, L, the positionposition-position box had $x, y, z$ dimensions of $\mathrm{L} \times \mathrm{L} \times 2 \mathrm{~L}$, where $\mathrm{L}$ corresponds to 512 pixels. We used this realization along with three sub-boxes with different $\mathrm{L}_{\mathrm{z}}$ to demonstrate how $\langle\sigma\rangle_{\text {nt }}$ and $\Delta \mathrm{V}_{\text {lsr }}$ depend on structure depth for a single turbulent realization. The original box had dimensions of $\mathrm{L} \times \mathrm{L} \times 2 \mathrm{~L}$ (the "double depth" box), the first sub-box was $\mathrm{L} \times \mathrm{L} \times \mathrm{L}$ (the "full depth" box), the second was $\mathrm{L} \times \mathrm{L} \times \mathrm{L} / 2$ (the "half depth" box), and the third was $\mathrm{L} \times \mathrm{L} \times \mathrm{L} / 4$ (the "quarter depth" box). For each of these sub-boxes (with uniform density), we created PPV cubes and then processed them similar to the observations. The end products were centroid velocity and velocity dispersion maps across the $\mathrm{L} \times \mathrm{L} x, y$ surface of each numerical box. We segmented each $\mathrm{L} \times \mathrm{L}$ surface into equally sized square regions with side $\mathrm{L}, \mathrm{L} / 2, \mathrm{~L} / 4, \mathrm{~L} / 8$, and calculated $\Delta \mathrm{V}_{\text {lsr }}$ and $\langle\sigma\rangle_{\text {nt }}$ within each segment to show how they scale with size. The results for each box are shown in Figure 24. Evidently, $\langle\sigma\rangle_{\mathrm{nt}}$ has no dependence on size, while $\Delta \mathrm{V}_{\mathrm{lsr}}$ increases with size, similar to the behavior seen in the observations in Figure 22, The size where $\Delta \mathrm{V}_{\mathrm{lsr}}$ crosses $\langle\sigma\rangle_{\mathrm{nt}}$ corresponds well with the depth of the numerical cube.

If turbulence in the observed region has similar behavior to that in the isotropic turbulent realization, then we can use the correlation of $\mathrm{V}_{\mathrm{lsr}}$ variation with size to estimate the depths of the Barnard 1 structures. For this correlation, we know the projected size is directly related to $\Delta \mathrm{V}_{\mathrm{lsr}}$, so there is no size ambiguity as there is in the correlation between projected size and $\langle\sigma\rangle_{\text {nt }}$. To estimate the typical depth of the cloud, we can find the size scale at which the best-fit line for $\Delta \mathrm{V}_{\mathrm{lsr}}$ versus projected size is equal to the best-fit line for $\langle\sigma\rangle_{\mathrm{nt}}$ versus projected size. This corresponds to $\sim 0.11 \mathrm{pc}$ for the $\mathrm{MCZ}$, and $\sim 0.18 \mathrm{pc}$ for the RSWZ. Under the assumption that turbulence is isotropic, this implies that Barnard 1 is on the order of 0.1-0.2 pc deep, comparable to the largest projected width $(\sim 0.2-0.3 \mathrm{pc})$ of individual structures.

Since the largest-scale dust structure in Barnard 1 extends over 1 pc on the sky, the overall region may be more flattened than spherical at the largest scales. Numerical simulations over the past 15 years have consistently shown that high-density sheets and filaments are a generic result of strongly supersonic turbulence with parameters comparable to those in observed clouds (e.g., reviews by Elmegreen \& Scalo 2004, Mac Low \& Klessen 2004, McKee \& Ostriker 2007). But measuring the depth of an individual structure in a molecular cloud is a difficult task. Comparison to realizations of turbulence suggests that the high angular resolution, large area observations from CLASSy allow us to estimate the physical depths of individual structures in molecular clouds, thus providing new observational insight into the true nature of those clouds 3 . Our observational result suggests that Barnard 1 is an over-dense region within the larger Perseus Molecular Cloud that could have formed a sheet-like geometry from supersonic turbulence. Lee et. al (2014, submitted) present the same size-linewidth relations using CLASSy observations of Serpens Main, and find similar behavior. We will compare the results from all our CLASSy regions in an upcoming cross-comparison paper.

\section{SUMMARY}

We presented the data release for the Barnard 1 region of the CARMA Large Area Star Formation Survey. CLASSy spectrally imaged over 800 total square arcminutes of the Perseus and Serpens Molecular Clouds at $7^{\prime \prime}$ angular resolution.

1. We spectrally imaged $\mathrm{N}_{2} \mathrm{H}^{+}, \mathrm{HCN}$, and $\mathrm{HCO}^{+}(J=1 \rightarrow 0)$ emission across 150 square arcminutes of Barnard 1. The final data products are position-position-velocity cubes with full line emission recovery

\footnotetext{
${ }^{3}$ We emphasize, however, that it is important to confirm the behavior seen in simple, isotropic turbulent realizations with fullyrealistic turbulent simulations of clouds. For best comparison to observations, it will be valuable to create synthetic PPV data cubes via radiative transfer modeling, rather than assuming uniform excitation and optically thin conditions.
} 

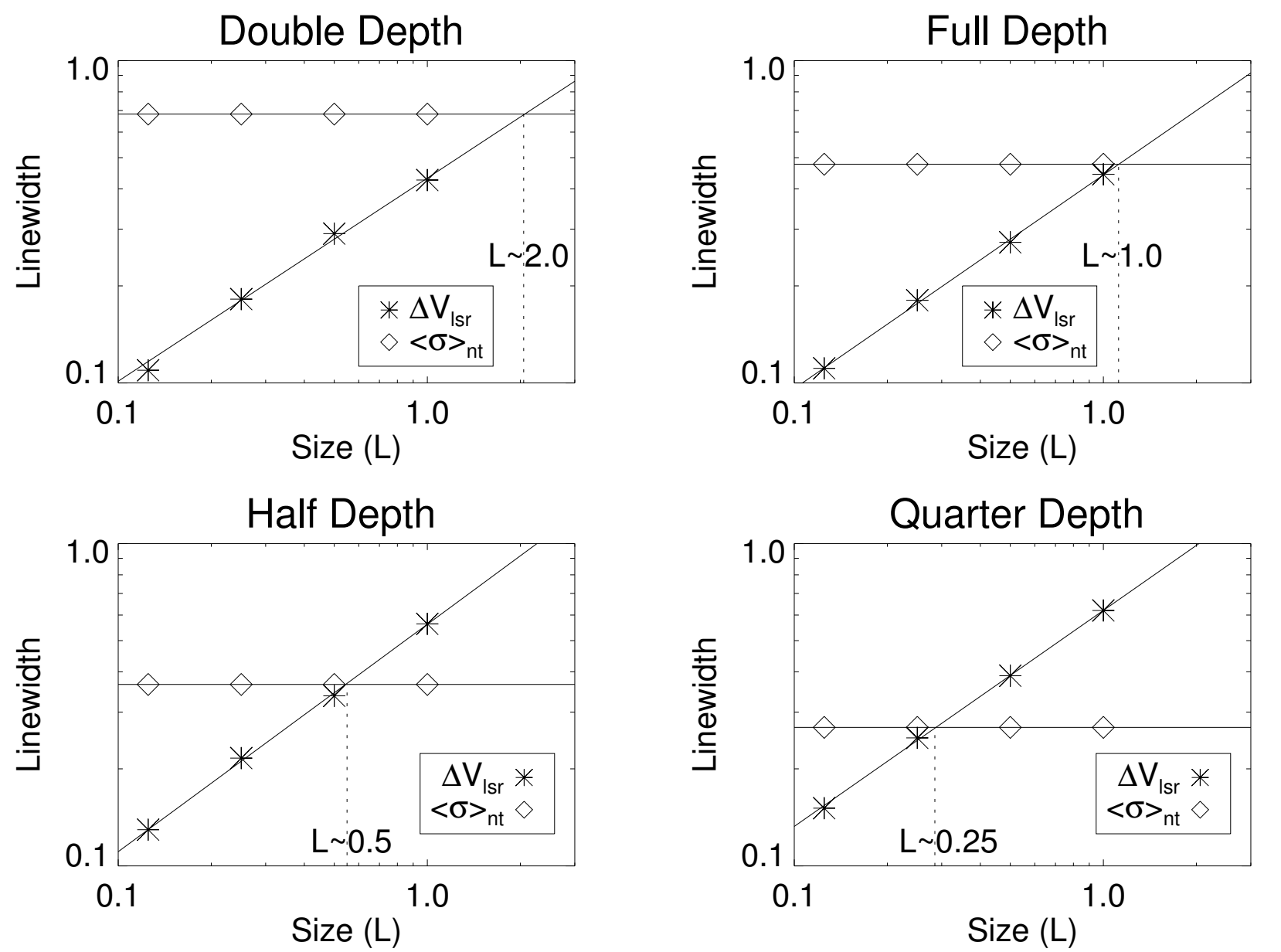

Fig. 24. - Size-linewidth relations from a numerical realization of an isotropic turbulent power spectrum. The four plots represent results from four different boxes taken from the realization: the "double depth" box is twice as deep as it is wide $(\mathrm{L} \times \mathrm{L} \times 2 \mathrm{~L})$, the "full depth" box is cubic $(\mathrm{L} \times \mathrm{L} \times \mathrm{L})$, the "half depth" box is half as deep as it is wide $(\mathrm{L} \times \mathrm{L} \times \mathrm{L} / 2)$, etc. The vertical axis represents the two linewidths we discussed in text: $\langle\sigma\rangle_{\mathrm{nt}}$ and $\Delta \mathrm{V}_{\mathrm{lsr}}$ (in arbitrary units). The horizontal axis represents the size-scale we sampled across the face of the box when calculating the linewidths: a size-scale of 1 represents sampling across the full $\mathrm{L} \times \mathrm{L}$ scale, a size-scale of 0.5 represents sampling across smaller-scale $\mathrm{L} / 2 \times \mathrm{L} / 2$ segments, etc. There are several things to notice in these panels: 1) $\langle\sigma\rangle_{\text {nt }}$ is independent of size, and its magnitude decreases by $\sqrt{2}$ as the box depth is halved from panel to panel, 2) $\Delta \mathrm{V}_{\text {lsr }}$ increases with increasing size, 3) The magnitude of $\langle\sigma\rangle_{\mathrm{nt}}$ and $\Delta \mathrm{V}_{\mathrm{lsr}}$ cross near the size that represents the depth of the box. 
obtained through joint deconvolution with single-dish observations. The velocity resolution of the data is $\sim 0.16 \mathrm{~km} \mathrm{~s}^{-1}$.

2. Four compact continuum sources were detected at $>5-\sigma$ at $3 \mathrm{~mm}$, all in the main core zone. B1-c, B1-b South, and B1-b North were previously known; we report a new detection of compact emission towards the Per-emb 30 continuum source.

3. The $\mathrm{N}_{2} \mathrm{H}^{+}(J=1 \rightarrow 0)$ gas morphology closely matches dust continuum observations of Herschel, while $\mathrm{HCN}$ and $\mathrm{HCO}^{+}(J=1 \rightarrow 0)$ emissions are weaker throughout most of the field and show less correlation with the long wavelength dust emission. $\mathrm{HCN}$ and $\mathrm{HCO}^{+}$also well-trace outflows in the main core zone.

4. Spectral line fitting of the molecular line data shows that the Barnard 1 main core is much more kinematically complex than the filaments and clumps that extend to its southeast; these filaments and clumps are characterized by more uniform centroid velocities and lower velocity dispersions.

5. We used dendrograms to identify $\mathrm{N}_{2} \mathrm{H}^{+}$gas structures in Barnard 1. The motivation for using dendrograms instead of a more traditional clumpfinding algorithm was the need to analyze the morphological and kinematic structure of dense gas across the wide range of spatial scales captured in our CLASSy data. We found that dendrograms are better able to quantify that range of spatial scales. We created a new, non-binary adaptation to the standard, binary dendrogram algorithm to ensure that the dendrograms represent the true hierarchy of the emission within the noise limits of real data, and that tree statistics can be used to quantify that hierarchy.

6. The non-binary dendrogram of Barnard 1 contains 41 leaves and 13 branches. We calculated three simple tree statistics using the dendrogram: the maximum branching level, the mean path length, and the mean branching ratio. The tree structure representing the dense gas around the main core is the most complex, with four hierarchy levels and the highest contrast leaves. The tree statistics give insight into the type and amount of fragmentation a region has undergone, and will be used to compare the hierarchical complexity of the different CLASSy regions.

7. We characterized the spatial properties of the dendrogram structures and derived structure sizes ranging from $\sim 0.01$ to $0.34 \mathrm{pc}$. The high angular resolution data reveal a variety of irregular shapes, showing that star-forming gas is not composed of well ordered spheroids and filaments on the smallest scales. We also characterized the kinematic properties of the structures and found that, in general, branches have larger $\mathrm{V}_{\text {lsr }}$ variation, but similar mean velocity dispersion, compared to the leaves. The gas surrounding the most massive compact continuum cores have the largest velocity dispersions in the entire region.

8. Using the spatial and kinematic properties of the dendrogram leaves and branches, we estimated the depth of the Barnard 1 cloud to be $\sim 0.1-0.2$ pc. This estimate was made by comparing two size-linewidth relations: one using the mean non-thermal velocity dispersion of the dendrogram objects, which is sensitive to the depth of the cloud, and the other using the $V_{\text {lsr }}$ variation of the objects, which is sensitive to the projected size of the cloud. The mean non-thermal velocity dispersion varied very little with structure size, while the $\mathrm{V}_{\text {lsr }}$ variation varied steeply with size. We interpreted this as an indication that Barnard 1 is more flattened than spherical on the largest scales. This method is a powerful tool for observationally probing the structure of molecular clouds into the plane of the sky.

The science-ready spectral line data cubes for the Barnard 1 region can be found through the online version of Figure 5. We will also host data products at our CLASSy website: http://carma.astro.umd.edu/classy. We welcome the community to make use of the data.

The authors would like to thank referee Alyssa Goodman for encouraging critiques that improved the paper, and all members of the CARMA staff that made these observations possible. CLASSy was supported 
by AST-1139990 (University of Maryland) and AST-1139950 (University of Illinois). Support for CARMA construction was derived from the Gordon and Betty Moore Foundation, the Kenneth T. and Eileen L. Norris Foundation, the James S. McDonnell Foundation, the Associates of the California Institute of Technology, the University of Chicago, the states of Illinois, California, and Maryland, and the National Science Foundation. Ongoing CARMA development and operations are supported by the National Science Foundation under a cooperative agreement, and by the CARMA partner universities.

Facility: CARMA

\section{REFERENCES}

André, P., Men'shchikov, A., Bontemps, S., Könyves, V., Motte, F., Schneider, N., Didelon, P., Minier, V., Saraceno, P., Ward-Thompson, D., di Francesco, J., White, G., Molinari, S., Testi, L., Abergel, A., Griffin, M., Henning, T., Royer, P., Merín, B., Vavrek, R., Attard, M., Arzoumanian, D., Wilson, C. D., Ade, P., Aussel, H., Baluteau, J.-P., Benedettini, M., Bernard, J.-P., Blommaert, J. A. D. L., Cambrésy, L., Cox, P., di Giorgio, A., Hargrave, P., Hennemann, M., Huang, M., Kirk, J., Krause, O., Launhardt, R., Leeks, S., Le Pennec, J., Li, J. Z., Martin, P. G., Maury, A., Olofsson, G., Omont, A., Peretto, N., Pezzuto, S., Prusti, T., Roussel, H., Russeil, D., Sauvage, M., Sibthorpe, B., Sicilia-Aguilar, A., Spinoglio, L., Waelkens, C., Woodcraft, A., \& Zavagno, A. 2010, A\&A, 518, L102

Ballesteros-Paredes, J., Klessen, R. S., Mac Low, M.-M., \& Vazquez-Semadeni, E. 2007, Protostars and Planets $\mathrm{V}, 63$

Beaumont, C. N., Offner, S. S. R., Shetty, R., Glover, S. C. O., \& Goodman, A. A. 2013, ApJ, 777, 173

Beckwith, S. V. W., Sargent, A. I., Chini, R. S., \& Guesten, R. 1990, AJ, 99, 924

Bergin, E. A. \& Tafalla, M. 2007, ARA\&A, 45, 339

Briggs, D. S., Schwab, F. R., \& Sramek, R. A. 1999, in Astronomical Society of the Pacific Conference Series, Vol. 180, Synthesis Imaging in Radio Astronomy II, ed. G. B. Taylor, C. L. Carilli, \& R. A. Perley, 127

Brunt, C. M. \& Mac Low, M.-M. 2004, ApJ, 604, 196

Chen, C.-Y. \& Ostriker, E. C. 2014, ApJ, 785, 69

Chen, X., Arce, H. G., Zhang, Q., Bourke, T. L., Launhardt, R., Jørgensen, J. K., Lee, C.-F., Foster, J. B., Dunham, M. M., Pineda, J. E., \& Henning, T. 2013, SMA Observations of Class 0 Protostars: A High Angular Resolution Survey of Protostellar Binary Systems

Crutcher, R. M. 2012, ARA\&A, 50, 29

di Francesco, J., Evans, II, N. J., Caselli, P., Myers, P. C., Shirley, Y., Aikawa, Y., \& Tafalla, M. 2007, Protostars and Planets V, 17

Dickman, R. L. \& Kleiner, S. C. 1985, ApJ, 295, 479

Elmegreen, B. G. \& Scalo, J. 2004, ARA\&A, 42, 211

Enoch, M. L., Evans, II, N. J., Sargent, A. I., \& Glenn, J. 2009, ApJ, 692, 973

Enoch, M. L., Young, K. E., Glenn, J., Evans, II, N. J., Golwala, S., Sargent, A. I., Harvey, P., Aguirre, J., Goldin, A., Haig, D., Huard, T. L., Lange, A., Laurent, G., Maloney, P., Mauskopf, P., Rossinot, P., \& Sayers, J. 2006, ApJ, 638, 293 
Evans, II, N. J., Dunham, M. M., Jørgensen, J. K., Enoch, M. L., Merín, B., van Dishoeck, E. F., Alcalá, J. M., Myers, P. C., Stapelfeldt, K. R., Huard, T. L., Allen, L. E., Harvey, P. M., van Kempen, T., Blake, G. A., Koerner, D. W., Mundy, L. G., Padgett, D. L., \& Sargent, A. I. 2009, ApJS, 181, 321

Fernández-López, M., Arce, H. G., Looney, L., Mundy, L. G., Storm, S., Teuben, P. J., Lee, K., Segura-Cox, D., Isella, A., Tobin, J. J., Rosolowsky, E., Plunkett, A., Kwon, W., Kauffmann, J., Ostriker, E., Tassis, K., Shirley, Y. L., \& Pound, M. 2014, ApJ, 790, L19

Goodman, A. A., Rosolowsky, E. W., Borkin, M. A., Foster, J. B., Halle, M., Kauffmann, J., \& Pineda, J. E. 2009, Nature, 457, 63

Hatchell, J., Richer, J. S., Fuller, G. A., Qualtrough, C. J., Ladd, E. F., \& Chandler, C. J. 2005, A\&A, 440, 151

Heyer, M. H. \& Brunt, C. M. 2004, ApJ, 615, L45

Hiramatsu, M., Hirano, N., \& Takakuwa, S. 2010, ApJ, 712, 778

Hirano, N., Kamazaki, T., Mikamxi, H., Ohashi, N., \& Umemoto, T. 1999, in Star Formation 1999, ed. T. Nakamoto, 181-182

Hirano, N., Kameya, O., Mikami, H., Saito, S., Umemoto, T., \& Yamamoto, S. 1997, ApJ, 478, 631

Hirota, T., Bushimata, T., Choi, Y. K., Honma, M., Imai, H., Iwadate, K., Jike, T., Kameya, O., Kamohara, R., Kan-Ya, Y., Kawaguchi, N., Kijima, M., Kobayashi, H., Kuji, S., Kurayama, T., Manabe, S., Miyaji, T., Nagayama, T., Nakagawa, A., Oh, C. S., Omodaka, T., Oyama, T., Sakai, S., Sasao, T., Sato, K., Shibata, K. M., Tamura, Y., \& Yamashita, K. 2008, PASJ, 60, 37

Hirota, T., Honma, M., Imai, H., Sunada, K., Ueno, Y., Kobayashi, H., \& Kawaguchi, N. 2011, PASJ, 63, 1

Houlahan, P. \& Scalo, J. 1992, ApJ, 393, 172

Huang, Y.-H. \& Hirano, N. 2013, ApJ, 766, 131

Jørgensen, J. K., Harvey, P. M., Evans, II, N. J., Huard, T. L., Allen, L. E., Porras, A., Blake, G. A., Bourke, T. L., Chapman, N., Cieza, L., Koerner, D. W., Lai, S.-P., Mundy, L. G., Myers, P. C., Padgett, D. L., Rebull, L., Sargent, A. I., Spiesman, W., Stapelfeldt, K. R., van Dishoeck, E. F., Wahhaj, Z., \& Young, K. E. 2006, ApJ, 645, 1246

Jørgensen, J. K., Johnstone, D., Kirk, H., \& Myers, P. C. 2007, ApJ, 656, 293

Kirk, H., Johnstone, D., \& Di Francesco, J. 2006, ApJ, 646, 1009

Larson, R. B. 1981, MNRAS, 194, 809

Li, H.-b., Goodman, A., Sridharan, T. K., Houde, M., Li, Z.-Y., Novak, G., \& Tang, K. S. 2014, ArXiv e-prints

Mac Low, M.-M. \& Klessen, R. S. 2004, Reviews of Modern Physics, 76, 125

Markwardt, C. B. 2009, in Astronomical Society of the Pacific Conference Series, Vol. 411, Astronomical Data Analysis Software and Systems XVIII, ed. D. A. Bohlender, D. Durand, \& P. Dowler, 251

Matthews, B. C., Hogerheijde, M. R., Jørgensen, J. K., \& Bergin, E. A. 2006, ApJ, 652, 1374

McKee, C. F. \& Ostriker, E. C. 2007, ARA\&A, 45, 565

Miesch, M. S. \& Bally, J. 1994, ApJ, 429, 645 
Ossenkopf, V. \& Mac Low, M.-M. 2002, A\&A, 390, 307

Pineda, J. E., Arce, H. G., Schnee, S., Goodman, A. A., Bourke, T., Foster, J. B., Robitaille, T., Tanner, J., Kauffmann, J., Tafalla, M., Caselli, P., \& Anglada, G. 2011, ApJ, 743, 201

Rebull, L. M., Stapelfeldt, K. R., Evans, II, N. J., Jørgensen, J. K., Harvey, P. M., Brooke, T. Y., Bourke, T. L., Padgett, D. L., Chapman, N. L., Lai, S.-P., Spiesman, W. J., Noriega-Crespo, A., Merín, B., Huard, T., Allen, L. E., Blake, G. A., Jarrett, T., Koerner, D. W., Mundy, L. G., Myers, P. C., Sargent, A. I., van Dishoeck, E. F., Wahhaj, Z., \& Young, K. E. 2007, ApJS, 171, 447

Rosolowsky, E. \& Leroy, A. 2006, PASP, 118, 590

Rosolowsky, E. W., Pineda, J. E., Foster, J. B., Borkin, M. A., Kauffmann, J., Caselli, P., Myers, P. C., \& Goodman, A. A. 2008a, ApJS, 175, 509

Rosolowsky, E. W., Pineda, J. E., Kauffmann, J., \& Goodman, A. A. 2008b, ApJ, 679, 1338

Sault, R. J., Teuben, P. J., \& Wright, M. C. H. 1995, in Astronomical Society of the Pacific Conference Series, Vol. 77, Astronomical Data Analysis Software and Systems IV, ed. R. A. Shaw, H. E. Payne, \& J. J. E. Hayes, 433

Solomon, P. M., Rivolo, A. R., Barrett, J., \& Yahil, A. 1987, ApJ, 319, 730

Teuben, P. J., Pound, M. W., Storm, S., Mundy, L. G., Salter, D. M., Lee, K., Kwon, W., Fernandez Lopez, M., \& Plunkett, A. 2013, in American Astronomical Society Meeting Abstracts, Vol. 221, American Astronomical Society Meeting Abstracts, 240.09

Williams, J. P., de Geus, E. J., \& Blitz, L. 1994, ApJ, 428, 693

\section{A. COMPARING CLUMP-FINDING AND DENDROGRAMS FOR CLASSy DATA}

We use the Cloudprops algorithm (Rosolowsky \& Leroy 2006) and an IDL dendrogram algorithm (Rosolowsky et al. 2008b to identify $\mathrm{N}_{2} \mathrm{H}^{+}$emission structures in our CLASSy data cubes. The goal is to compare clumpfinding and dendrogram object identification methods when applied to nearby star forming regions observed with high angular resolution. Both algorithms analyze the emission in position-position-velocity cube to segment the emission into structures. Figure 25 shows Cloudprops (top) and dendrogram (bottom) contours for identified structures, overplotted on two $\mathrm{N}_{2} \mathrm{H}^{+}$velocity channels around the B1-b continuum cores. The common color contours represent the same structures in the left and right panels. There are clearly major differences in the number and shapes of the identified structures between the two methods.

We find that the Cloudprops algorithm forces emission into small-scale clumps, even when the data does not show clear regions with strong intensity enhancements. When visually inspecting the emission channels in Figure 25, there is no physical reason to think that the plateau of emission west of the B1-b cores is made up on several, independent clumps of dense gas that are separated with orderly borders. The technical reason for the clumpy breakdown is that Cloudprops first locates the highest level, local closed isocontours. Next, the algorithm steps down in intensity dividing the lower intensity emission between the stronger peaks according to a nearest-neighbor algorithm. This division of lower intensity emission across a fairly uniform emission plateau leads to the arbitrary borders seen in the top row of Figure 25. Cloudprops and other clump-finding algorithms

This preprint was prepared with the AAS IATEX macros v5.2. 
work best for sparse fields that have resolved separations between objects-identifying giant molecular clouds in an extragalactic source is one example of where they work well. But attempting to decompose nearby, well-resolved, molecular gas emission into many small clumps with fairly straight borders does not have good physical motivation. Similar conclusions were found in Goodman et al. (2009), where the authors showed that CLUMPFIND (Williams et al. 1994) makes artificial attempts to fill in the structure between meaningful clumps.

Instead of partitioning all of the emission into individual clumps according to a nearest-neighbor scheme, it makes more physical sense to interpret it as lower intensity structure surrounding the peaks within it. For example, the peaks could represent gas that fragmented from the lower intensity emission surrounding them. This scenario considers that small-scale features can form from existing large-scales features, and is a hierarchical way of thinking about the data. If we accept that molecular clouds are hierarchical, then this is a more physical way to interpret the emission, and it requires an algorithm that can track the emission structure as a function of contour level intensity - a dendrogram algorithm does just that.

How does a dendrogram algorithm work? We quickly review the basic dendrogram procedure using a one-dimensional emission profile seen in Figure 26 (from Rosolowsky et al. 2008b). (See that paper for more discussion of this example and the extension to two- and three-dimensions.) A one-dimensional, horizontal contour can be started at the absolute maximum of the emission profile and stepped downward to the base of emission. During this process, the peaks and merge levels of the three local maxima are identified and represented in a dendrogram (seen inset within the emission profile and repeated on the right with labels).

A dendrogram is composed of leaves and branches. In our example, the three leaves of the dendrogram correspond to the three local maxima in the emission profile that do not break up into any substructure. The peak intensity of a leaf represents the peak intensity of its corresponding local maximum, while the lowest intensity of a leaf represents the intensity where the corresponding local maximum merges with another local maximum. The two branches of the dendrogram correspond to lower-intensity emission that breaks up into substructure at higher intensities. The upper branch represents the lower-intensity emission that seeds the two strongest leaves, and the lower branch represents even lower-intensity emission that encompasses all of the emission peaks. The peak intensity of a branch represents the intensity where the leaves above it merge together, while the lowest intensity of a branch represents the intensity where it merges with another leaf or branch (the case of the left branch) or where it reaches the lowest measured intensity (the case of the lower branch). This algorithm can be extended from one-dimension to two- and three-dimensions to operate on position-position or position-position-velocity datasets. All of our object identification for CLASSy clouds was done in three-dimensions, even if we occasionally represent the identified objects as two-dimensional contours for visualization simplicity.

Going back to our example in Figure 25, the dendrogram identified objects in the two $\mathrm{N}_{2} \mathrm{H}^{+}$channels are shown on the bottom row of the figure. Two peaks of emission (leaves) are identified with isolated contours (green and blue contours), and then get joined at a lower intensity level within a larger scale contour (a branch shown as the cyan contour).

To summarize, the dendrogram identification method is more appropriate for our data and science goals. Our data capture small-scale dense gas structure due to the high-angular resolution of the interferometer, and they capture large-scale dense gas features because of the large-area mosaicing and full emission reconstruction with single-dish data. Our science goals involve studying how small-scale gas features connect to the largescale cloud features. The dendrogram approach lets us investigate the physical and kinematic properties of the dense gas across the wide range of spatial scales probed by our CLASSy data in a way that purely small-scale, clumpfind-like segmentation would not allow. 


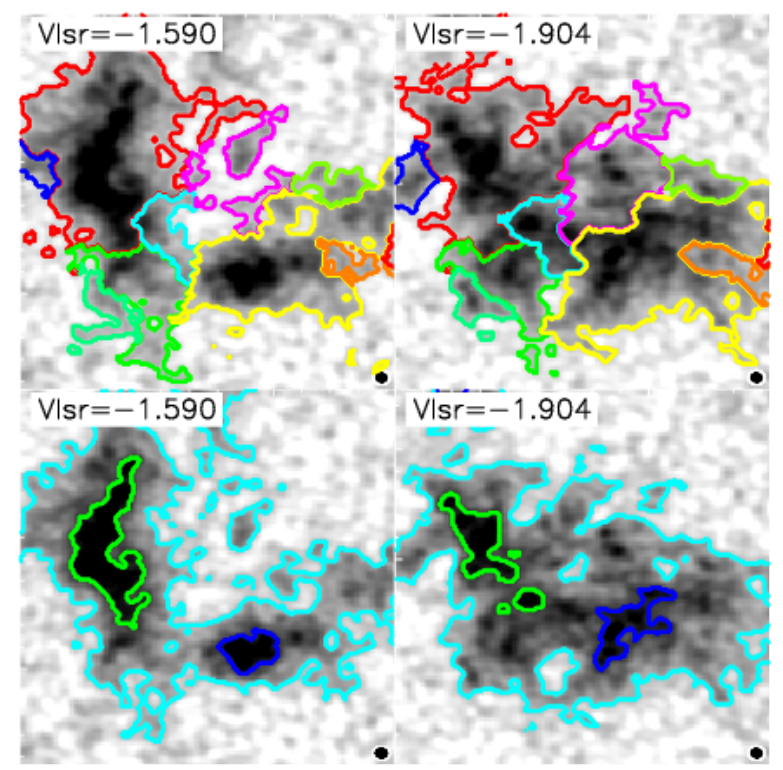

Fig. 25. - Two $\mathrm{N}_{2} \mathrm{H}^{+}$channel maps of the B1-b region for a comparison of Cloudprops (top) and dendrogram (bottom) object identification results. Each Cloudprops and dendrogram object is contoured with a unique color-this particular region is broken up into nine distinct Cloudprops objects, and three dendrogram objects. Cloudprops tends to put all emission into smaller-scale, independent clumps, while dendrograms identifies peaks of emission as leaves (green and blue contours in the online version), and then identifies lower intensity contour(s) that surround them as branches (cyan contour in the online version). We argue that the dendrogram approach is the more appropriate one for high angular resolution studies of nearby molecular clouds, since it captures the hierarchical nature of the gas emission across the wide range of spatial scales that exists in those regions.
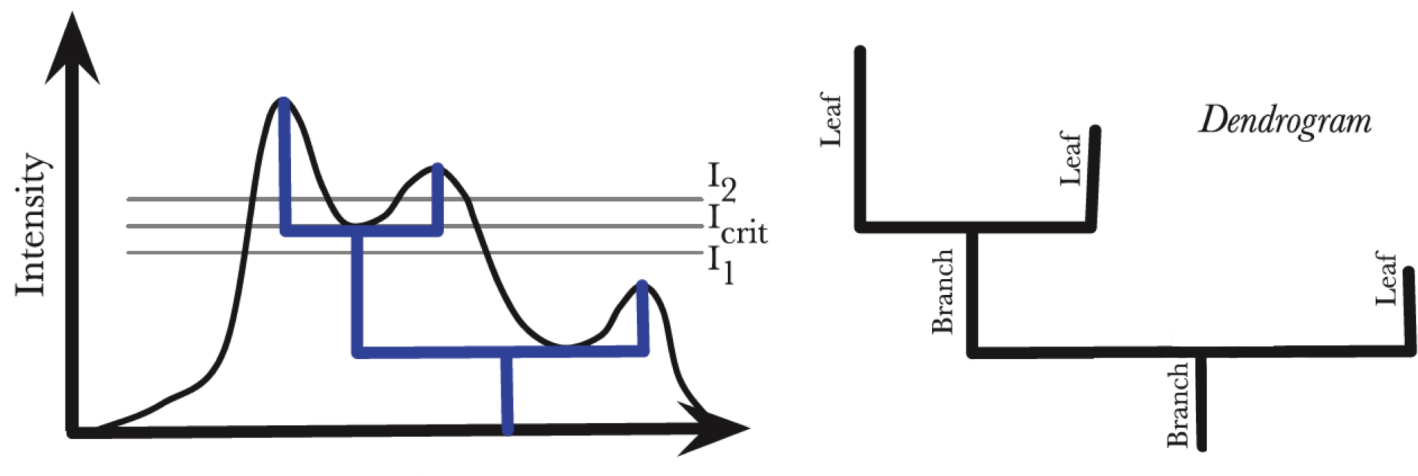

Position

Fig. 26. - This figure is taken from Rosolowsky et al. (2008b). The left image shows a one-dimensional, three-peak emission profile with the dendrogram representation as an inset. The dendrogram is reproduced on the right with the tree features labeled (the labels differ slightly from Rosolowsky et al. (2008b) to accommodate the naming conventions used in this paper). The dendrogram is formed by keeping track of the emission structure at a function of intensity level. For example, contouring the emission profile at the $I_{1}$ level produces a single object, while contouring at $I_{2}$ produces two objects - the $I_{\text {crit }}$ contour represents the level where the two strongest peaks in the emission profile merge together. In the dendrogram, this contour level is represented by a horizontal line connecting the two strongest leaves into the branch. Readers should see the original paper for a detailed discussion on dendrograms in all three dimensions. 


\section{B. A NEW NON-BINARY DENDROGRAM PROCEDURE}

The standard dendrogram algorithm forms a purely binary tree - thinking about a tree growing from the base upward, every branch terminates in either two leaves, a leaf and a branch, or two branches. A single branch is not allowed to directly sprout three leaves, even if the leaves merge into the branch at exactly the same level. In this three leaf example, two of the leaves would get merged into a branch, and then that branch would merge with the remaining leaf into another branch. We refer to this as "phantom branching" because an artificial branch was introduced only to enforce a binary merger requirement, as opposed to being motivated by the true nature of the data being modeled. This makes interpreting the true hierarchical nature of the data difficult, and makes a quantitative assessment of the dendrogram using tree statistics impossible (see Houlahan \& Scalo (1992) for the use of tree statistics to interpret the hierarchical nature of emission). Going back to our example, we set out to allow all three leaves to merge into a single branch - we set out to allow non-binary mergers.

Non-binary dendrograms are useful for two reasons: 1) they provide a collection of branches that respect the noise inherent in real data, and 2) they allow the user to quantitatively compare the hierarchical complexity of different emission regions, and correlate that complexity with properties of those regions (e.g., star formation efficiency, column density, etc.).

The three-leaf example we used to motivate non-binary dendrograms is artificial; it is extremely unlikely for a real dataset to have three leaves merging at exactly the same intensity. Typical behavior seen in real data sets may have two leaves merge into a branch at the $1.0 \mathrm{Jy} \mathrm{beam}^{-1}$ level, and then merge with a third leaf into another branch at the $0.98 \mathrm{Jy}$ beam $^{-1}$ level. Our key argument is that these three leaves should merge into a single branch, if the sensitivity of the dataset is lower than the branching difference. For example, the sensitivity of our Barnard 1 dataset is $0.13 \mathrm{Jy} \mathrm{beam}^{-1}$, and the standard dendrogram algorithm produces branching in increments less than $0.13 \mathrm{Jy}_{\text {beam }}{ }^{-1}$ to ensure binary mergers - this produces a collection of branches that is not meaningful within the noise limits of the data, and limits the quantitative interpretation of the hierarchy. Our new method alleviates the binary merger requirement and allows branches to sprout an unrestricted amount of leaves and/or branches.

The key technical differences between our new non-binary method and the standard method are: 1) we restrict branching to intensity steps equal to integer values of the 1- $\sigma$ sensitivity of the data, instead of allowing branching at infinitely small intensity steps, 2) we have an algorithm that can cluster more than two objects into a single group, instead of being restricted to clustering two objects at a time. Another way to think about the difference is that the standard dendrogram code yields the one emission hierarchy that represents the one realization of the noise applied to the true emission being observed. Our modified dendrogram code instead represents an observable emission hierarchy within the noise limits of the data. 


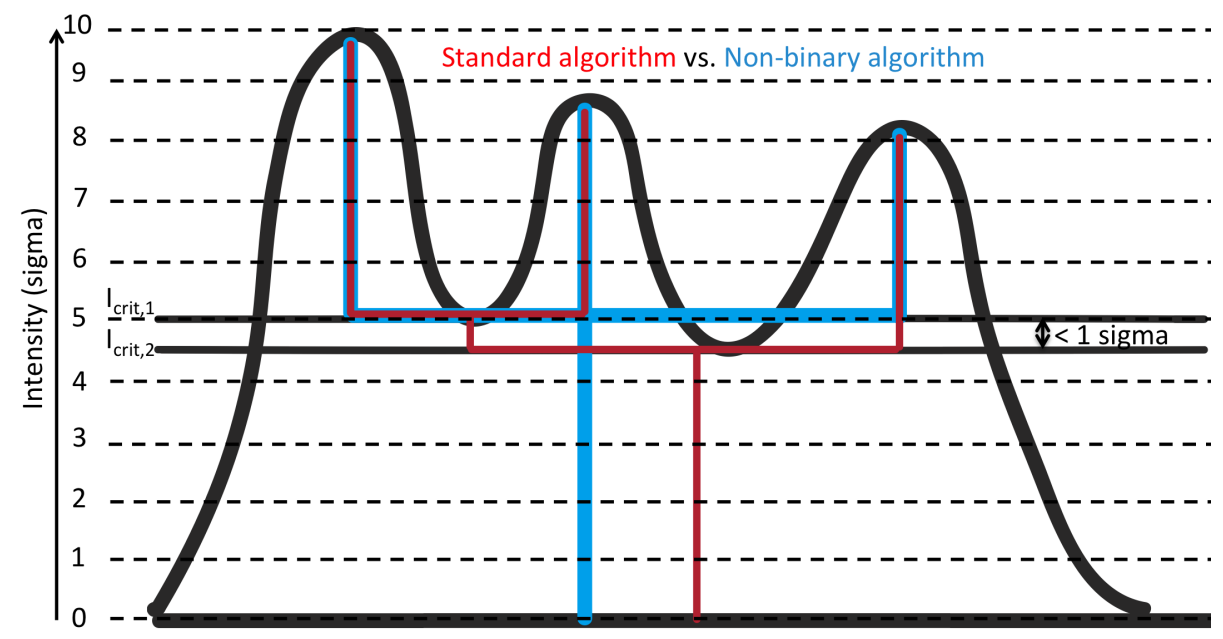

Fig. 27. - Cartoon example of the difference between the standard dendrogram algorithm, and our new non-binary algorithm. The standard algorithm (red tree in the online version) forces binary mergers of dendrogram leaves and branches- the left and center peaks are first merged into a branch at the $\mathrm{I}_{\text {crit, } 1}$ contour level, and that branch is then merged with the right peak at the $\mathrm{I}_{\text {crit,2 }}$ contour level. Since the two merger levels are separated by less than the 1-sigma sensitivity of the data, we argue that the branch between $I_{c r i t, 1}$ and $I_{c r i t, 1}$ is not motivated by the data, but by the requirement of binary mergers. Our new non-binary algorithm (cyan in the online version) merges all three peaks at the highest merger level, and assigns the remaining emission between $I_{c r i t, 1}$ and $I_{c r i t, 2}$ to a single branch. This represents an observable emission hierarchy within the noise limits of the data.

A cartoon example of the difference is shown in Figure 27. The cartoon shows a one-dimensional intensity profile, where the intensity axis is quoted in terms of the sigma sensitivity units of the data. Under the standard algorithm (red tree), the two leftmost peaks merge together at the level of the first saddle point $\left(\mathrm{I}_{\text {crit, }, 1}\right.$, which represents an intensity level of 5-sigma) into a branch. The algorithm then merges that first branch with the rightmost peak at the level of the second saddle point $\left(\mathrm{I}_{\text {crit, } 2}\right.$, which is less than 1-sigma lower than $\left.\mathrm{I}_{\text {crit,1 }}\right)$ into a second branch. Our algorithm (cyan tree) merges all three peaks into a single branch at the 5-sigma level since the two saddle points are separated by less than the 1-sigma sensitivity of the data.

Our algorithm comes with one obvious caveat. The resulting dendrogram depends on the $1-\sigma$ contouring of the data; if branching levels are shifted slightly higher or lower, then the dendrogram can change. For example, consider a dataset in Kelvin units (for visual simplicity), with $0.10 \mathrm{~K}$ sensitivity, two leaves that peak at $4.00 \mathrm{~K}$ and merge together at $1.00 \mathrm{~K}$, and a third leaf that peaks at $3.00 \mathrm{~K}$ and merges with the other leaves at $0.98 \mathrm{~K}$. Our algorithm would set $1-\sigma$ branching levels in $0.10 \mathrm{~K}$ steps starting from the $4.00 \mathrm{~K}$ peak, which means that $1.00 \mathrm{~K}$ is an available merge level. All three leaves would be merged into a single branch at $1.00 \mathrm{~K}$ using our non-binary algorithm. But if the peak intensity is $3.98 \mathrm{~K}$ instead of $4.00 \mathrm{~K}$, then $1.00 \mathrm{~K}$ is not an available branching level anymore - but $1.08 \mathrm{~K}$ and $0.98 \mathrm{~K}$ are. For that case, the two strongest leaves would merge at $1.08 \mathrm{~K}$, and then merge with the third leaf at $0.98 \mathrm{~K}$. This simple example shows how our dendrograms, and the tree statistics derived from them, can be slightly altered by shifts in the 1- $\sigma$ contouring of the data. We went from a dendrogram with one branching level, a branching ratio of three, and a mean path length of one, to a dendrogram with to two branching levels, a mean branching ratio of two, and a mean path length of 1.66.

This caveat produces only small changes in the dendrogram and tree statistics when tested on the actual Barnard 1 data presented in the paper. We shifted the 1- $\sigma$ branching levels by $0.33-\sigma$; compared to the results presented in section 6 , the mean branching ratio changed from 3.9 to 3.8 , and the mean path length and maximum branching level were unchanged. We argue that the benefits of this new non-binary algorithm outweigh this caveat, particularly when science goals include: 1) correlating the physical properties of several star forming regions with differences in dendrogram structure (by comparing tree statistics of different regions, which is not possible with the standard algorithm), and 2) comparing the structure and kinematics of large-scale 
and small-scale structures within a single region (by comparing the spatial and kinematic properties of leaves and branches, which is more difficult for the end-user if the branch list is contaminated by phantom branching from the standard algorithm). 